\title{
Breast cancer associated muscle fatigue: Novel targets to improve survival and quality of life
}

\author{
Hannah Elizabeth Wilson \\ West Virginia University, hewilson@mix.wvu.edu
}

Follow this and additional works at: https://researchrepository.wvu.edu/etd

Part of the Cancer Biology Commons

\section{Recommended Citation}

Wilson, Hannah Elizabeth, "Breast cancer associated muscle fatigue: Novel targets to improve survival and quality of life" (2020). Graduate Theses, Dissertations, and Problem Reports. 7785.

https://researchrepository.wvu.edu/etd/7785

This Dissertation is protected by copyright and/or related rights. It has been brought to you by the The Research Repository @ WVU with permission from the rights-holder(s). You are free to use this Dissertation in any way that is permitted by the copyright and related rights legislation that applies to your use. For other uses you must obtain permission from the rights-holder(s) directly, unless additional rights are indicated by a Creative Commons license in the record and/ or on the work itself. This Dissertation has been accepted for inclusion in WVU Graduate Theses, Dissertations, and Problem Reports collection by an authorized administrator of The Research Repository @ WVU.

For more information, please contact researchrepository@mail.wvu.edu. 
Breast cancer associated muscle fatigue:

Novel targets to improve survival and quality of life

Hannah E. Wilson

Dissertation submitted

to the College of Medicine

at West Virginia University

in partial fulfillment of the requirements for the degree of

Doctor of Philosophy in

Cancer Cell Biology

\author{
Lori Hazlehurst, Ph.D., Chair \\ Wei Du, Ph.D. \\ John Hollander, Ph.D. \\ Malcolm Mattes, M.D. \\ Elena Pugacheva, Ph.D. \\ Emidio E. Pistilli, Ph.D., Advisor
}

Morgantown, West Virginia

2020

Keywords: breast cancer, cachexia, fatigue, patient-derived orthotopic xenograft, peroxisome-proliferator activated receptors

Copyright 2020 Hannah E. Wilson 


\title{
ABSTRACT \\ Breast cancer associated muscle fatigue: Novel targets to improve survival and quality of life
}

\author{
Hannah E. Wilson
}

Women diagnosed with breast cancer $(\mathrm{BC})$ currently represent the largest group of cancer survivors in the United States, accounting for over $20 \%$ of those living after a cancer diagnosis. Most BC survivors report an unusual degree of fatigue, which can present prior to diagnosis and continue for many years after treatment cessation. Recent studies show that deficits in muscle function predict shorter survival in cancer, perhaps due to the fact that fatigue is known to reduce a patient's tolerance to anticancer therapies. Therefore, improving muscle function in BC patients has the potential to improve both quality of life and survival in the most commonly diagnosed cancer type. At present, few therapies for cancer-related fatigue show clinical efficacy, and the pathogenesis of this condition is largely unknown. This work aimed to identify molecular changes induced in skeletal muscle by BC, using patient samples and by developing novel in vitro and in vivo model systems. We tested the hypothesis that BC induces dysregulation of metabolic pathways regardless of molecular subtype or treatment history. Transcriptomic and proteomic analyses in BC patient muscle samples indicated mitochondrial dysfunction, which was evident across all molecular subtypes of BC and unrelated to patients' treatment histories. In vitro and in vivo experimentation confirmed the ability of $\mathrm{BC}$ cells to alter mitochondrial respiration and ATP content in muscle tissue via a secreted factor without the involvement of immune or stromal cell mediators. These data indicate that BC-induced fatigue is related to defects in skeletal muscle mitochondrial function induced directly by BC cells, and that targeting mitochondrial 
function or mitochondrial density in muscle could be a viable therapeutic strategy to improve both survival and quality of life in BC survivors. 


\section{TABLE OF CONTENTS}

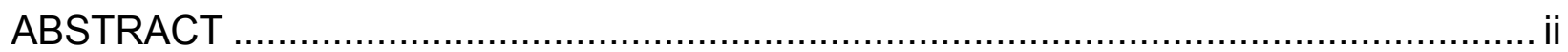

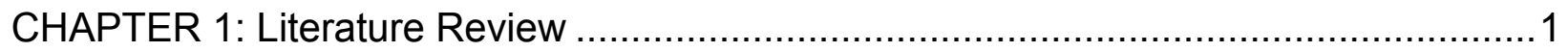

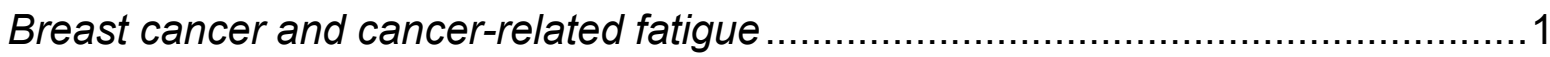

Cancer cachexia and its definitional ambiguity ...............................................

Animal models of cancer cachexia and cancer-related fatigue ............................. 4

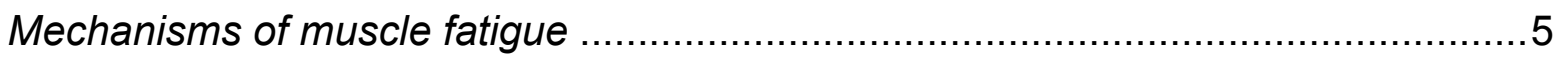

Peroxisome-proliferator activated receptors ...................................................

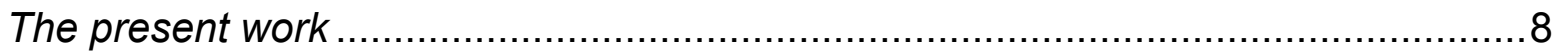

CHAPTER 2: Human breast cancer xenograft model implicates peroxisome proliferatoractivated receptor signaling as driver of cancer-induced muscle fatigue .....................10 CHAPTER 3: Skeletal muscle reprogramming by breast cancer regardless of treatment

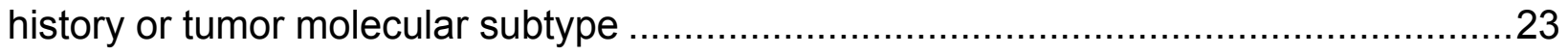

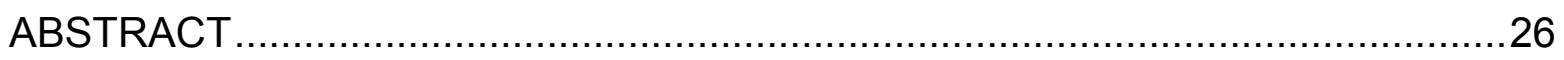

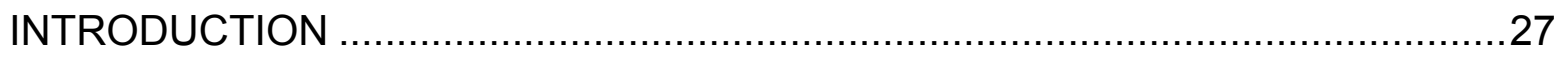

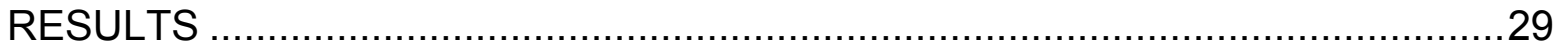

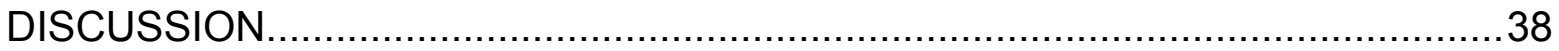

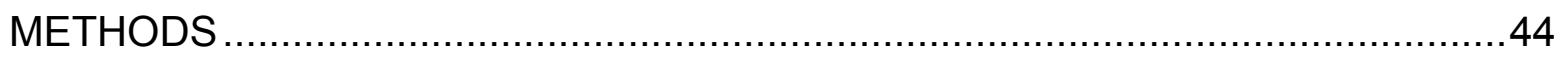

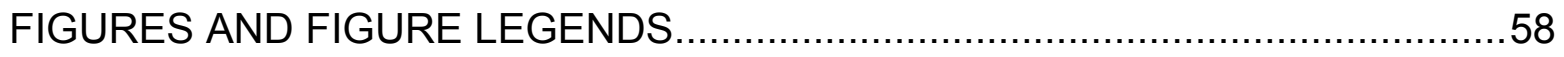

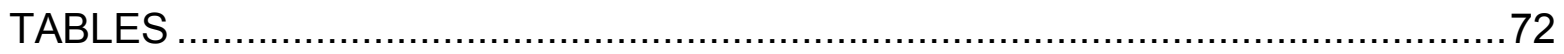




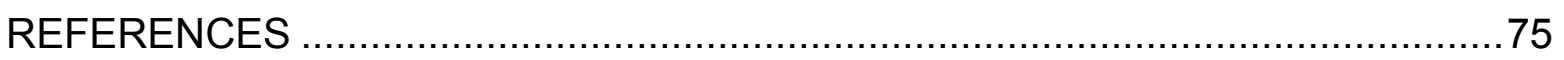

CHAPTER 4: Causal mediation analysis of muscle wasting on the muscle fatigue response to experimentally-induced breast cancer ............................................... 82

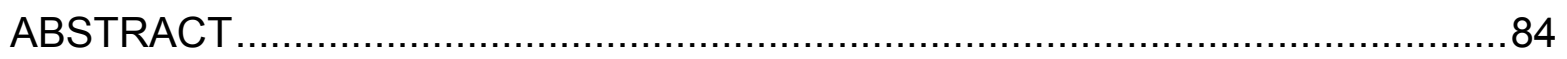

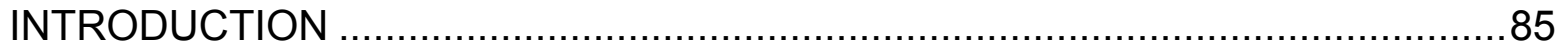

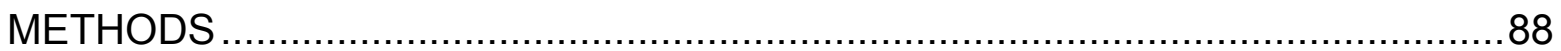

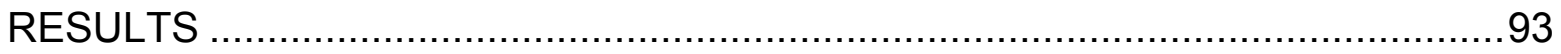

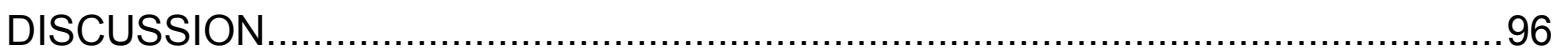

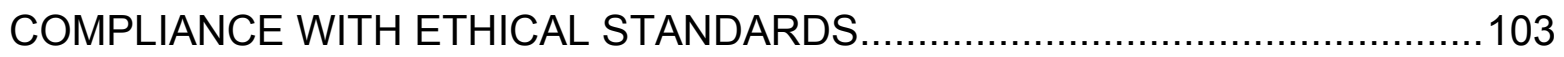

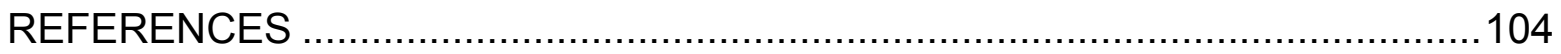

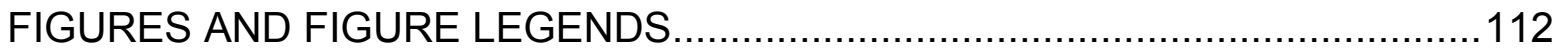

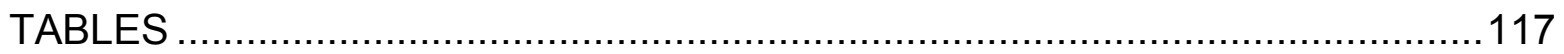

CHAPTER 5: Breast cancer-associated skeletal muscle mitochondrial dysfunction and lipid accumulation is reversed by PPARG ..........................................................118

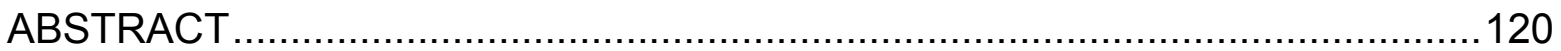

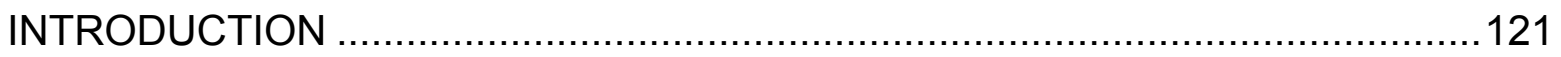

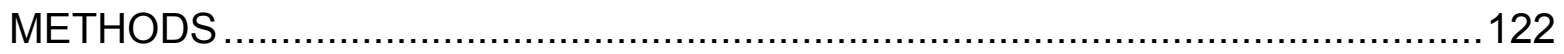

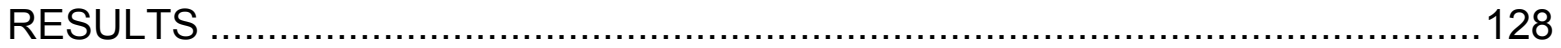

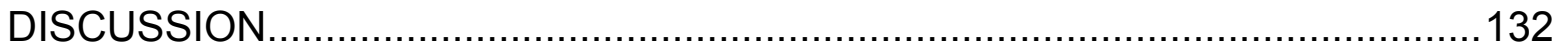

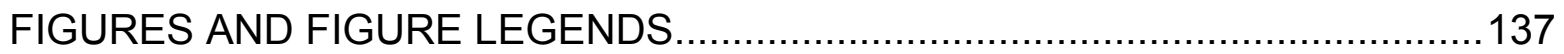




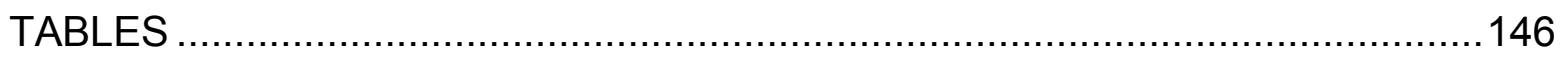

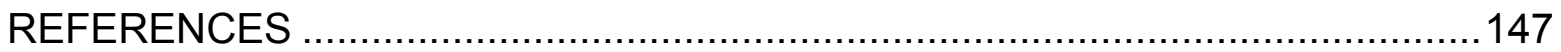

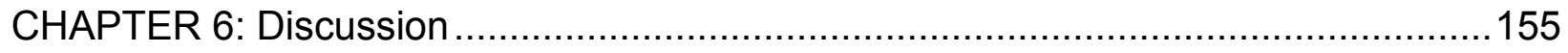

Development of and insights from the BC-PDOX model of BC-related fatigue. ....155

Clinical correlates of skeletal muscle molecular profiles in BC..........................157

In vitro modeling of BC-induced skeletal muscle dysfunction .............................159

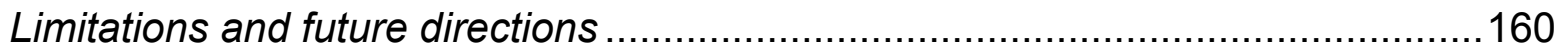

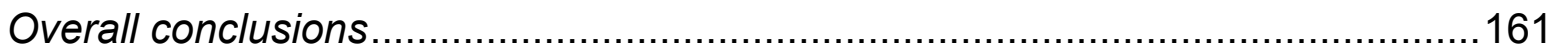

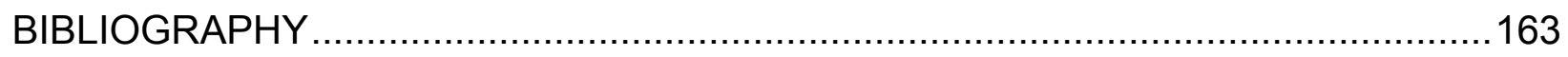


CHAPTER 1: Literature Review

\section{Breast cancer and cancer-related fatigue}

Breast cancer $(\mathrm{BC})$ is currently the most commonly diagnosed cancer in the United States, affecting 1 in 8 women in their lifetime ${ }^{1}$. Advances in BC treatment have led to significant advances in overall survival in BC and other cancer types in recent decades 2,3 , and $90 \%$ of women diagnosed with BC are now expected to live at least 5 years after diagnosis ${ }^{4}$. In many cases, cancer can now be thought of as a chronic disease requiring long-term management, including addressing the numerous contributors to diminished quality of life associated with this disease ${ }^{5}$. One such contributor to diminished quality of life in $B C$ is the problem of cancer-related fatigue (CRF), which affects the vast majority of women diagnosed with $B C{ }^{6-9}$. CRF can present in $B C$ patients prior to the initiation of anti-cancer treatments ${ }^{10,11}$, is commonly exacerbated by anti-cancer therapies ${ }^{7,9,12,13}$, and frequently affects women years after treatment cessation ${ }^{7,9,14}$. It is now established that deficits in muscle function are poor prognostic indicators in cancer, perhaps because muscle atrophy and fatigue are known to reduce a patient's physical and psychological tolerance for anti-cancer therapies ${ }^{9,15-19}$. Therefore, targeting muscle function in $\mathrm{BC}$ patients could improve both quality of life and survival in $\mathrm{BC}$, and this intervention would have the potential to impact millions of women currently living with BC.

Management of $\mathrm{CRF}$ is a multifactorial endeavor due to the multifactorial nature of CRF. Physicians are recommended to, to the best of their ability, identify the relative contributors to $\mathrm{CRF}$ and target these factors individually ${ }^{20}$. These contributory factors include anemia, immunological responses to tumor growth or tumor cell death, 
hormonal and metabolic disturbances, and emotional distress, among other factors ${ }^{20}$, ${ }^{21}$. Large scale interventional trials for CRF are severely lacking, likely due to the multifactorial nature of CRF and the difficulty that complexity presents in identifying an appropriate study population for a given intervention ${ }^{20,22}$. While several of the contributory factors to CRF do have appropriate targeted therapies, such as selective serotonin reuptake inhibitors for depression and iron supplementation for iron deficiency anemia, CRF still remains an unmet need in clinical oncology according to patients themselves. In fact, over $60 \%$ of respondents in a recent large-scale survey of patients with cancer reported experiencing fatigue that they felt was not adequately addressed by their medical providers ${ }^{23}$. This disparity between symptom burden and therapeutic options should be addressed to provide the ever-growing cadre of cancer survivors longer and more enjoyable lives.

\section{Cancer cachexia and its definitional ambiguity}

The problem of cancer-associated cachexia, where individuals with cancer lose a significant portion of their bodyweight and also experience a decline in physical function 12, 24 , has gained increasing attention in recent years. So much so that reversal of cancer cachexia was named a "Provocative Question" by the National Cancer Institute for 2020 , a designation that confers additional funding avenues to researchers addressing these questions ${ }^{25}$. The exact definition of cancer cachexia has been in a state of flux over the last twenty years. Most generally agree that for a person to be cachectic, they must exhibit a significant degree of weight loss, and that weight loss must not be simply due to decreased caloric intake and must be associated with a disease ${ }^{12,24,26}$. The recommended degree of weight loss required for a cachexia 
diagnosis varies among experts, and increasing evidence suggests that different thresholds may be needed for different patient populations ${ }^{27}$, though an international group of clinicians and cancer cachexia researchers recently recommended $5 \%$ body weight loss as the threshold for a cachexia diagnosis ${ }^{24,28}$. However, cachexia is not only a weight loss syndrome; it also commonly culminates in metabolic abnormalities and decline in physical function. This acknowledgment has led to experts calling for the inclusion of functional and metabolic endpoints in interventional studies ${ }^{29}$. Further, in some clinical contexts, cachexia does not confer a survival deficit until $8 \%$ or even $10 \%$ bodyweight loss has occurred, and conversely, weight-stable patients experiencing cachexia-associated symptoms have decreased overall survival relative to other patients ${ }^{27,30,31}$. These data suggest that the definition of cachexia may need to be specific for disease type or patient population, and that weight loss, sarcopenia, and cachexia-related symptoms may need to be considered differently in different clinical contexts. In general though, studies correlating overall survival with these various definitions of cachexia unambiguously show that cachexia is a poor prognostic indicator ${ }^{28}$, with an even worse prognosis if patients are experiencing functional deficits or severe weight loss ${ }^{26,31}$.

Cachexia presenting with advanced disease has been described by physicians for millennia ${ }^{32}$, but its mechanisms remain elusive and few therapeutic avenues have exhibited clinical success ${ }^{20}$. It is commonly assumed that deficits in skeletal muscle function among individuals with cancer are caused by the muscle wasting observed in cachexia, and therefore by preventing muscle wasting, muscle function will be preserved. While this assumption is largely supported by data in men with cancer, 
women seem to exhibit a unique phenotype in that they can exhibit cancer-related deficits in muscle function, including decreased strength and increased fatigability, in the absence of muscle atrophy ${ }^{33-36}$. This sexual dimorphism is important to acknowledge for several reasons. First, clinicians should be aware that a woman may be experiencing the symptoms associated with cachexia when she does not appear frankly cachectic, with this being particularly important given that less than $5 \%$ of women with BC have any cachexia-related International Classification of Disease code in their electronic medical records ${ }^{37}$. Additionally, it is unlikely that therapies focusing on the maintenance or acquisition of muscle mass will be effective in reducing CRF in women with cancer. And finally, studies addressing cancer cachexia and CFR should study men and women separately given the obvious sexual dimorphism in physiological responses to cancer. Many interventional studies in cancer cachexia have shown negative results, but these may be related to problems with study design, specifically in grouping men and women together ${ }^{38-40}$, in grouping multiple cancer types together ${ }^{38-41}$, or in using only muscle mass or overall body weight as the primary endpoint without also assessing muscle function ${ }^{39,42}$. Multiple large scale interventional studies have reported improvements in muscle mass without concomitant improvements in muscle functional parameters or quality of life scores, further emphasizing the need to assess muscle function in addition to mass ${ }^{43}$.

\section{Animal models of cancer cachexia and cancer-related fatigue}

Animal studies seeking to understand cancer-related fatigue exhibit similar issues in study design. Because the definition of cachexia depends on the patient exhibiting significant weight loss, all animal models used to study cancer cachexia exhibit this 
phenotype ${ }^{44}$. Even many animal models developed with the express purpose of studying cancer-related or treatment-related fatigue exhibit weight loss ${ }^{45,46}$. These models are not appropriate for studying $\mathrm{CRF}$ in $\mathrm{BC}$, where the majority of women experience an unusual degree of fatigue despite remaining weight stable. Thus, a significant goal of the work reported herein was the identification and study of a model that adequately recapitulated the experience of $\mathrm{BC}$ patients, who describe an increased susceptibility to fatigue in the absence of cachexia. To address this goal, we have extensively characterized the muscle physiology response to BC in the patient-derived orthotopic xenograft (PDOX) model, as the PDOX model has been previously shown to reproduce human disease histology and progression better than cell line-based assays 47.

\section{Mechanisms of muscle fatigue}

There are two major categories of mechanisms that contribute to pathological fatigue: central fatigue, involving neural transmission within the brain and spinal cord, and peripheral fatigue, which encompasses the actual motor units within muscle tissue, their ability to respond to nervous stimulation, and factors that mediate this relationship such as ATP availability and ion concentrations in various skeletal muscle compartments. It is likely that both of these categories contribute to the fatigue experienced by women with $\mathrm{BC}$, but the scope of this research is limited to peripheral mechanisms of fatigue. These include the coordinated transport of $\mathrm{Na}^{+}$and $\mathrm{K}^{+}$involved in cellular depolarization and repolarization in response to nervous stimulation, release of $\mathrm{Ca}^{2+}$ from the sarcoplasmic reticulum in response to membrane depolarization, calcium-dependent cross-bridge cycling, ATP-dependent reuptake of $\mathrm{Ca}^{2+}$ back into the sarcoplasmic reticulum to allow 
for relaxation and succeeding contraction, and the metabolic processes that govern high-energy phosphate availability, which is required for several of the aforementioned activities. ATP availability is governed by several processes, including transfer of highenergy phosphate from phosphocreatine to AMP to form ATP in the creatine phosphate shuttle system, generation of ATP in the tricarboxcylic acid cycle, and generation of ATP by oxidative phosphorylation in the mitochondria. All of these activities have a variety of regulatory mechanisms and therefore a multitude of factors can contribute to ATP availability and therefore alter susceptibility to fatigue within skeletal muscle ${ }^{48}$, let alone the multitude of muscle-extrinsic factors that are beyond the scope of this project. Muscle size is governed by a different but partially overlapping list of factors, but is predominantly governed by the catabolism and anabolism of muscle structural components in response to the contractile demands placed upon the muscle. Thus, muscle disuse can lead to significant muscle atrophy, and this is frequently reported in advanced disease where patients' mobility is restricted. While the ability to carry out activities of daily living can be impacted by both fatigue and muscle atrophy and fatigue can lead to muscle disuse and subsequent atrophy, fatigue resistance is not related to muscle size and therefore reversing muscle atrophy is unlikely to reverse the increased susceptibility to fatigue experienced by BC patients. Further, ignoring the mechanisms that contribute to fatigue while seeking to reverse atrophy may be counterproductive, as fatigue can limit a patient's ability to exercise and can therefore limit the contractile stimuli required to induce muscle hypertrophy. 


\section{Peroxisome-proliferator activated receptors}

The peroxisome-proliferator activated receptors are a family of lipid sensing, ligandactivated transcription factors that regulate multiple metabolic processes across numerous tissue types. In particular, they play a large role in regulation of lipid metabolism, with various isoform and tissue specificities. The PPARa isoform exhibits higher expression levels in tissues with high rates of fatty acid catabolism, including the liver, skeletal muscle, and cardiac muscle. The PPARy isoform is largely specific for adipose tissue, is considered a master regulator of adipogenesis, and modulates insulin sensitivity, though low levels of expression are present in skeletal muscle ${ }^{49}$. The PPARy isoform, in skeletal muscle in particular, likely plays a significant role in wholebody metabolic regulation because the muscle-specific PPARy knockout mouse exhibits marked insulin resistance, hyperlipidemia, hepatosteatosis, and increased fat mass despite no difference in overall bodyweight relative to age-matched controls ${ }^{50}$. The PPAR $/ \delta$ isoform is not particularly well characterized despite being ubiquitously expressed ${ }^{49}$. It is apparent that this family of nuclear receptors aid in regulating whole body energy homeostasis, and modulating these transcription factors pharmacologically has significantly benefited patients with various metabolic diseases, including diabetes, dyslipidemia, and atherosclerosis ${ }^{51}$. Intriguingly, the use of PPAR agonists in preclinical mouse models of Lewis lung carcinoma ${ }^{52}$, colon adenocarcinoma ${ }^{53}$, and nonsmall cell lung cancer ${ }^{54}$ has shown promising efficacy for cancer cachexia. Thus, it is reasonable to suppose a mechanistic role for this family of transcription factors in the metabolic derangements occurring in CRF, as well as in the treatment of this debilitating syndrome. 
The present work

The work presented herein has focused on three major points. First, we aimed to develop and characterize a mouse model that recapitulates the human BC phenotype of increased muscle fatigability in the absence of muscle atrophy. Our identification of the BC-PDOX model as such a model is a finding of paramount importance to the field, as it will allow for focused investigation into this clinically common phenotype. We then aimed to utilize the PDOX model to identify potential targetable mechanisms of muscle fatigability using comparative transcriptomic analysis, utilizing muscle samples from PDOX-bearing animals and women with $\mathrm{BC}$ and correlating gene expression changes with patients' clinical information. This avenue yielded several interesting target pathways, with a leading candidate being dysregulation of mitochondrial function, perhaps due to the apparent repression of PPAR signaling. Following up on this potential target, we aimed to develop in vitro model systems for studying BC-induced skeletal muscle dysfunction, choosing to utilize a conditioned media model. We have been largely successful in developing such a model, showing that factors released from BC cells induce significant metabolic changes in differentiated cultured muscle cells, including repression of aerobic activity, induction of intramyocellular lipid accumulation, and repression of PPAR-mediated transcription. Overexpression and pharmacological modulation of PPAR-gamma significantly altered these phenotypes in skeletal muscle, confirming that the PPAR signaling pathway is indeed linked to the mechanism by which BC-derived factors produce metabolic changes in muscle cells. Overall, our data support a paradigm of $\mathrm{BC}$-induced fatigue resulting from skeletal muscle metabolic dysfunction secondary to PPAR repression, suggesting that targeting mitochondrial 
function via the PPAR signaling pathway could be a viable therapeutic strategy to improve BC-induced muscle fatigue. 
CHAPTER 2: Human breast cancer xenograft model implicates peroxisome proliferatoractivated receptor signaling as driver of cancer-induced muscle fatigue

This journal article describes the first animal model system to recapitulate the human BC phenotype of increased muscle fatigability in the absence of muscle atrophy, using the patient-derived orthotopic xenograft model of BC (BC-PDOX) ${ }^{55}$. Here, we show that the BC-PDOX model exhibits the expected phenotype of increased susceptibility to fatigue in an ex vivo system without muscle atrophy, and we utilize RNA-sequencing technology to compare transcriptomic changes occurring in muscle from $\mathrm{BC}$ patients to those occurring in tumor-bearing animals. Using these technologies, we identify the peroxisome-proliferator activated receptors as potential targets to treat $\mathrm{BC}$-induced muscle fatigue.

In this project, I conducted the majority of the bioinformatics and statistical analyses, conducted all qRT-PCR and Western blotting experiments reported in the manuscript, assisted with collection of muscle physiology data, and was the main contributor to the manuscript text. 
Human Breast Cancer Xenograft Model Implicates

Peroxisome Proliferator-activated Receptor Signaling as Driver of Cancer-induced Muscle Fatigue

\author{
Hannah E. Wilson 1,2 , Kacey K. Rhodes ${ }^{2,3}$, Daniel Rodriguez ${ }^{4}$, Ikttesh Chahal ${ }^{5}$, \\ David A. Stanton ${ }^{5}$, Joseph Bohlen ${ }^{5}$, Mary Davis ${ }^{6}$, Aniello M. Infante ${ }^{7}$, \\ Hannah Hazard-Jenkins ${ }^{8}$, David J. Klinke ${ }^{9}$, Elena N. Pugacheva ${ }^{2,3}$, and \\ Emidio E. Pistilli $i^{2,5,9,10}$
}

\begin{abstract}
Purpose: This study tested the hypothesis that a patientderived orthotopic xenograft (PDOX) model would recapitulate the common clinical phenomenon of breast cancer-induced skeletal muscle (SkM) fatigue in the absence of muscle wasting. This study additionally sought to identify drivers of this condition to facilitate the development of therapeutic agents for patients with breast cancer experiencing muscle fatigue.

Experimental Design: Eight female BC-PDOX-bearing mice were produced via transplantation of tumor tissue from 8 female patients with breast cancer. Individual hind limb muscles from BC-PDOX mice were isolated at euthanasia for RNA-sequencing, gene and protein analyses, and an ex vivo muscle contraction protocol to quantify tumor-induced aberrations in SkM function. Differentially expressed genes (DEG) in the BC-PDOX mice relative to control mice were identified
\end{abstract}

MD/PhD Medical Scientist Program, West Virginia University School of Medicine, Morgantown, West Virginia. ${ }^{2}$ Cancer Institute, West Virginia University School of Medicine, Morgantown, West Virginia. ${ }^{3}$ Department of Biochemistry, West Virginia University School of Medicine, Morgantown, West Virginia. ${ }^{4}$ Statler College of Engineering and Mineral Resources, West Virginia University, Morgantown, West Virginia. ${ }^{5}$ Division of Exercise Physiology, Department of Human Performance, West Virginia University School of Medicine, Morgantown, West Virginia. ${ }^{6}$ Department of Physiology, Pharmacology and Neuroscience, West Virginia University School of Medicine, Morgantown, West Virginia. ${ }^{7}$ Genomics Core Facility, West Virginia University, Morgantown, West Virginia. ${ }^{8}$ Department of Surgery, West Virginia University School of Medicine, Morgantown, West Virginia. ${ }^{9}$ Department of Microbiology, Immunology, and Cell Biology, West Virginia University School of Medicine, Morgantown, West Virginia. ${ }^{10}$ West Virginia Clinical and Translational Sciences Institute, West Virginia University School of Medicine, Morgantown, West Virginia.

Note: Supplementary data for this article are available at Clinical Cancer Research Online (http://clincancerres.aacrjournals.org/).

Current address for J. Bohlen: Lieber Institute for Brain Development, Johns Hopkins Medical School, Baltimore, Maryland.

Corresponding Author: Emidio E. Pistilli, West Virginia University School of Medicine, Morgantown, WV 26506. Phone: 304-293-0291; Fax: 304-293-7105; E-mail: epistilli2@hsc.wvu.edu

doi: 10.1158/1078-0432.CCR-18-1565

(C2018 American Association for Cancer Research. using DESeq2, and multiple bioinformatics platforms were employed to contextualize the DEGs.

Results: We found that SkM from BC-PDOX-bearing mice showed greater fatigability than control mice, despite no differences in absolute muscle mass. PPAR, mTOR, IL6, IL1, and several other signaling pathways were implicated in the transcriptional changes observed in the BC-PDOX SkM. Moreover, 3 independent in silico analyses identified PPAR signaling as highly dysregulated in the SkM of both BC-PDOX-bearing mice and human patients with early-stage nonmetastatic breast cancer.

Conclusions: Collectively, these data demonstrate that the BC-PDOX model recapitulates the expected breast cancer-induced SkM fatigue and further identify aberrant PPAR signaling as an integral factor in the pathology of this condition.

\section{Introduction}

Cachexia has been clinically recognized as a consequence of advanced cancer for millennia (1) and has been recognized as a cause of death in a significant portion of patients with cancer for nearly 100 years (2). Despite this long history, the mechanistic underpinnings of this devastating condition remain poorly understood, and no curative therapeutics exist (3). A complicating factor in the treatment of cancer cachexia is its multifactorial nature that defies an easy definition, but most agree that cachexia includes some combination of weight loss, inflammation, and abnormal metabolism $(4,5)$. A multifactorial treatment approach is needed to treat this syndrome (6-9), and that approach must consider the potentially disparate pathologies contributing to weight loss, muscle wasting, and muscle fatigue (10).

SkM dysfunction in patients with cancer is often considered a consequence of muscle wasting. However, a large percentage of women with breast cancer report fatigue (11-14) despite $<20 \%$ of patients with breast cancer experiencing significant weight loss, and $<5 \%$ having any cachexia-related International Classification of Disease code on their electronic medical records (15). Although muscle strength and fatigue are strongly related to muscle mass in male patients with cancer, this does not appear to be the case in women. Studies in multiple cancer types indicate female 


\section{Translational Relevance}

Cancer-associated skeletal muscle fatigue is a common problem in clinical oncology that is often associated with cancer cachexia, but is not exclusively observed in cachectic patients. The majority of patients with breast cancer report muscle fatigue despite cachexia being relatively rare in this patient population, especially in patients with nonmetastatic disease. The clinically relevant phenotype of muscle fatigue in the absence of frank cachexia has no established model system and no approved therapeutic agents. Here, we utilize a breast cancer patient-derived orthotopic xenograft (BC-PDOX) model to recapitulate the human phenotype of tumor-induced muscle fatigue without muscle wasting. Bioinformatics analyses via multiple platforms identifies PPAR signaling as central to transcriptional alterations observed in skeletal muscle from both BC-PDOX-bearing mice and human patients with breast cancer. These data suggest that pharmacologic agents targeting PPAR isoforms, such as FDA-approved thiazolidinediones (TZDs), may be of clinical benefit to patients with breast cancer experiencing muscle fatigue.

patients with cancer lose muscle quality without losing muscle mass (16-18). This sexual dimorphism in susceptibility to muscle wasting versus muscle dysfunction must be acknowledged given that women with advanced cancer have significantly lower incidence of cachexia than men (19-21). One cannot conclude that weight-stable women are immune to the quality-of-life deficits of cancer-induced muscle dysfunction. Elucidating the mechanistic underpinnings of the clinically relevant phenotype of cancerinduced muscle dysfunction in female patients could provide a great quality-of-life benefit to patients with breast cancer and survivors. A first step toward this goal includes the establishment of a model system that recapitulates the clinically relevant phenotype of cancer-induced muscle dysfunction in the absence of muscle wasting.

A significant barrier to progress in the field of cancer cachexia is the lack of a diverse set of model systems that adequately reflect the heterogeneity of human responses to tumor growth. The use of spontaneous or engrafted tumor models in inbred mouse strains enforces a degree of uniformity in the laboratory that does not exist in the clinic. Patient-derived orthotopic xenograft (PDOX) models, wherein a human tumor fragment is implanted into the appropriate anatomic location of an experimental animal, offer a significant improvement over established cell line-based grafts in their increased ability to reproduce human disease histology and progression (22). As the PDOX model recapitulates human disease histology, progression, and metastatic burden, it is reasonable to hypothesize that $\mathrm{BC}$-PDOX mice will recapitulate the common clinical phenomenon of breast cancer-induced SkM fatigue in the absence of muscle wasting.

In this study, we characterized the transcriptional alterations and assessed multiple physiologic parameters induced in SkM of female mice bearing BC-PDOXs. RNA sequencing (RNA-seq) and subsequent bioinformatics analyses indicated aberrations in canonical pathways previously implicated in cancer cachexia, along with multiple novel pathways that have not yet been related to this phenomenon. Correlation of the BC-PDOX model with our previously published gene expression profile of SkM of human patients with breast cancer strongly implicates PPAR signaling in the pathophysiology of breast cancer-induced muscle fatigue (23). To the authors' knowledge, this study represents the first characterization of cancer-induced alterations in the SkM transcriptome in the context of a BC-PDOX model, as well as the first successful attempt to generate a model of breast cancerinduced muscle dysfunction in the absence of muscle wasting. Collectively, these data demonstrate that BC-PDOX tumor growth recapitulates the expected breast cancer-induced muscle fatigue and further identify aberrant PPAR signaling as an integral factor in the pathology of this condition.

\section{Materials and Methods}

\section{Patient selection}

Breast cancer tumor tissue was procured at West Virginia University (WVU) Cancer Institute (Morgantown, WV) and by the NCI Cooperative Human Tissue Network under approved WVU Institutional Review Board protocol and WVU Cancer Institute Protocol Review and Monitoring Committee. Informed written consent was obtained from each subject or each subject's guardian. Individuals could be included in this study if they (i) were deemed to have operable disease or were undergoing a diagnostic biopsy, (ii) had been diagnosed with, or were suspected to have, invasive adenocarcinoma of the breast, (iii) were over 21 years old, and (iv) provided informed consent. Muscle biopsies were obtained from patients with breast cancer and controls as previously reported (23). Conduct of research involving human patients at West Virginia University (Morgantown, WV) is guided by the principles set forth in the Ethical Principles and Guidelines for the Protection of Human Subjects of Research (Belmont Report) and is performed in accordance with the Department of Health and Human Services policy and regulations at 45 CFR 46 (Common Rule).

\section{Establishment of BC-PDOX models}

Animal experiments were approved by the WVU Institutional Animal Care and Use Committee. BC-PDOX models were produced by implanting freshly collected breast tumor tissue into the cleared mammary fat pad of NOD.CG-Prkd $d^{s c i d} I l 2 r^{t m 1 ~} \mathrm{Wjl}^{\mathrm{j}} / \mathrm{SzJ} /$ 0557 (NSG) mice (Fig. 1A) as described previously $(24,25)$. Briefly, female NSG mice were anesthetized with isoflurane. An incision was made over the lower lateral abdominal wall, a 3-mm pocket was opened under the skin to expose the mammary fat pads, and a single tumor fragment of $2 \mathrm{~mm}^{3}$ was placed into the pocket. Tumor growth was assessed by veterinary staff based on approved WVU Tumor Development and Monitoring Policy.

BC-PDOX-bearing mice were euthanized by $\mathrm{CO}_{2}$ asphyxiation upon reaching a maximal tumor burden score, or earlier if exhibiting signs of health distress. BC-PDOX tumors were then removed from the passage number 0 (P0) animals and cut into fragments, which were then frozen and, in part, passaged into naïve NSG animals to produce passage number 1 (P1) PDOX. Experimental animals bearing BC-PDOX (P0-2) were euthanized at the same humane endpoint as P0 animals. Mice were housed at $22^{\circ} \mathrm{C}$ under a 12-hour light/12-hour dark cycle and received food and water ad libitum. Each BC-PDOX model produced was authenticated with original patient's biopsy using genomic DNA and short tandem repeat-based PCR amplification (Arizona University Genomics Core Facility, Tucson, AZ). Routine human and mouse pathogen screening was performed on original and 
Wilson et al.

A Human tumor fragment obtained from patient with breast cancer

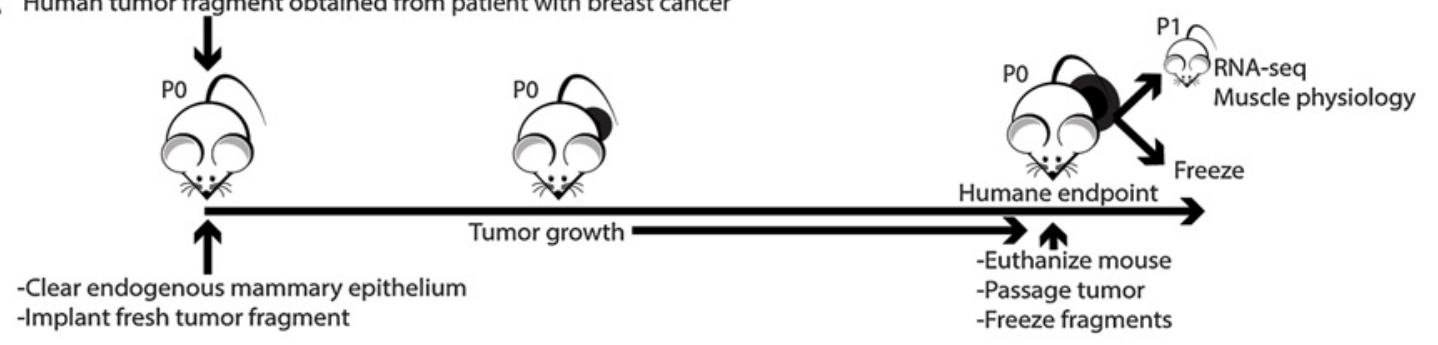

B

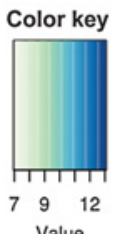

Value
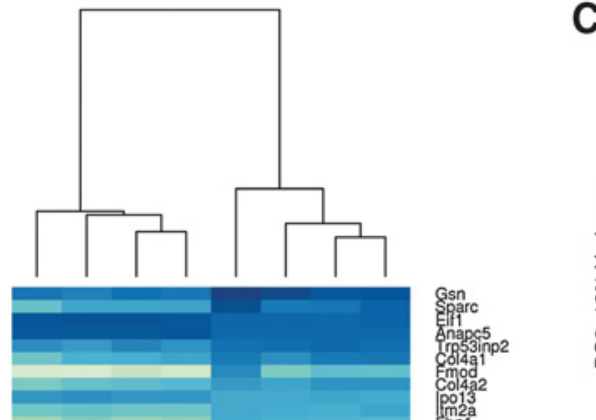

C

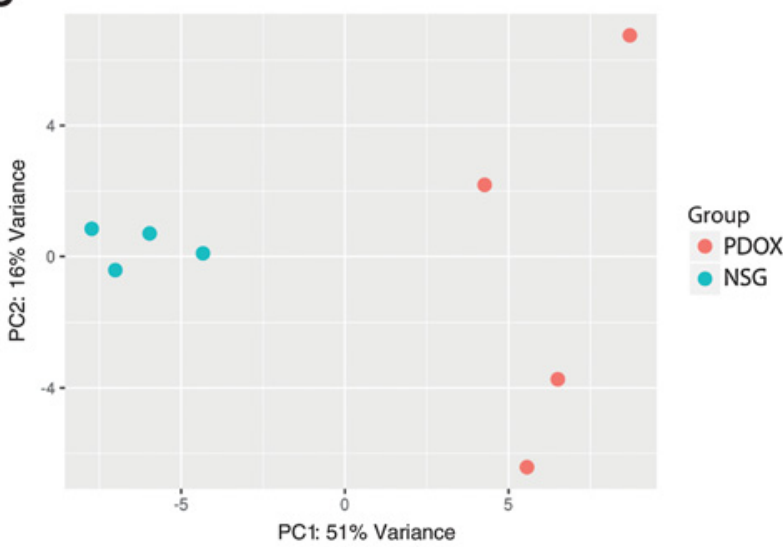

Figure 1.

Establishment of BC-PDOX model. Analysis of SkM from BC-PDOX mice compared with NSG controls indicates widespread transcriptional reprogramming of SkM transcriptome in response to tumor growth. A, Schematic diagram outlining establishment of BC-PDOX models (PO) and passaging of tumor to generate study animals (PO-P2). B, Normalized gene expression heatmap showing differential expression patterns of the 50 most differentially expressed genes between BC-PDOX $(n=4)$ and NSG control mice $(n=4)$ organized according to unsupervised clustering analysis. C, Principal component analysis showing that transcriptional profiles of NSG and BC-PDOX SkM cluster separately, indicating that tumor growth induces transcriptional alterations in SkM.

passaged tissue by Charles River Laboratories. RNA sequence and whole-exome analysis of original biopsy and passage 1 BC-PDOX further confirm relatedness of samples and stability of genomic alterations identified in patient with breast cancer and corresponding PDOX. Expression of clinically relevant breast cancer receptors correlate with the original breast cancer biopsy. BC-PDOX mice $(n=8)$ were compared with nontumor NSG mice $(n=6)$ as well as NSG mice that received human breast cancer-PDOXs that did not engraft (PDOX-Con; $n=5$ ).

\section{E0771 syngeneic mammary tumor model}

Detailed methods for E0771 mouse mammary tumor cell growth in syngeneic C57BL/6 mice have been described previously (23). Briefly, $1 \times 10^{6}$ E0771 cells were suspended in sterile PBS and orthotopically implanted into the fourth inguinal mammary fat pad on the left side of experimental mice. Three arms of the study included uninjected control mice (Con; $n=8)$, mice euthanized following 2 weeks of tumor growth $(2 \mathrm{WK}, n=5)$, and mice euthanized following 4 weeks of tumor growth (4WK, $n=11$ ). E0771 cells were obtained through an MTA (Wake Forest University Health Sciences; July 2014). Cells were authenticated and screened for the presence of contaminants prior to use (IDEXX Bioresearch). Experiments described in this publication using E0771 cells were completed between January 2015 and June 2016.

\section{RNA isolation, sequencing, and bioinformatics}

Total RNA was isolated from gastrocnemius muscles of experimental mice ( $n=4$ /group) and pectoralis major muscles from human patients (23) using TRIzol (Thermo Fisher Scientific) and established methods (26). RNA purity was assessed using a NanoDrop spectrophotometer, with 260/280 readings of at least 
2.0. RNA quantity was measured via a qubit fluorometer. RNA integrity was measured on an Agilent bioanalyzer with an RNA Nano chip. RNA samples had RNA Integrity Numbers (RIN) $>9$, indicating high-quality RNA. Libraries for RNA-seq were constructed and DEGs were identified according to previously published methods (23). RNA-Seq libraries were built using the Stranded mRNA kit from KAPA Biosciences with Illumina compatible adapters. The concentrations of the completed libraries were quantified with a qubit fluorometer using high-sensitivity DNA reagent. Libraries were subsequently run on the bioanalyzer using a high-sensitivity DNA chip to determine average fragment size. Completed libraries were then pooled in equimolar concentrations and sequenced on 1 lane of the HiSeq 1500 with PE50 bp reads (BioProject ID: PRJNA496042; murine samples). Subsequently, data were compared with the GRCm38.84 reference genome from Ensembl. There was no alignment step utilized. However, Salmon was used for quantification, with both gcBias and seqBias set, and libType A (27). Salmon output was imported into DESeq using tximport (28). DESeq was run with default parameters with the exception of a filtering step: genes which did not have a least 3 samples with a count of at least 10 were eliminated. RNA-Seq output was analyzed using various bioinformatics platforms: STRING (29), Enrichr (30), Ingenuity Pathway Analysis (IPA; Qiagen Inc., https://www.qiagenbioinformatics.com/ products/ingenuity-pathway-analysis/), and GeneAnalytics (31). Values presented from Enrichr analyses represent those obtained via querying the intersecting set of transcription factors' target genes identified by both the Encyclopedia of DNA Elements (ENCODE) and ChIP Enrichment Analysis (ChEA).

\section{Protein isolation}

Protein homogenates were made from tibialis anterior muscles of experimental mice ( $n=4$ or 5/group), pectoralis major muscles from human patients with breast cancer $(n=15)$, and control female patients undergoing breast surgery $(n=5)$ using a $5-\mathrm{mL}$ Wheaton Tissue Grinder (DWK Life Sciences Inc.) in a tissue lysis buffer $(20 \mathrm{mmol} / \mathrm{L}$ Tris $\mathrm{HCl}(\mathrm{pH}=7.4), 150 \mathrm{mmol} / \mathrm{L} \mathrm{NaF}$, $1 \mathrm{mmol} / \mathrm{L}$ EDTA, $1 \%$ Triton X-100, 10\% glycerol, $1 \mathrm{mmol} / \mathrm{L}$ $\mathrm{NaO}_{3} \mathrm{~V}$ ) with $1 \times$ Pierce Protease and Phosphatase Inhibitor (Thermo Fisher Scientific). Homogenates were cleared via brief centrifugation, and protein concentration was quantified using Pierce Coomassie Plus Protein Assay (Thermo Fisher Scientific) according to the manufacturer's protocol.

\section{Western blotting analysis}

Protein homogenates were diluted to a final concentration of $1 \mu \mathrm{g} \cdot \mu \mathrm{L}^{-1}$ in $1 \mathrm{X}$ NuPAGE LDS Sample Buffer (Thermo Fisher Scientific). Fifteen to $20 \mu \mathrm{g}$ of total protein was loaded per well, resolved in NuPAGE Novex 4\%-12\% Bis-Tris Gels (Thermo Fisher Scientific). Proteins were transferred to nitrocellulose membrane, blocked for 1 hour in $1 \times$ TBS, $0.1 \%$ Tween $20,5 \%$ BSA followed by incubation with primary antibody overnight at $4^{\circ} \mathrm{C}$. Membranes were then washed thrice in TBS $+0.1 \%$ Tween 20 prior to application of appropriate secondary antibody (Thermo Fisher Scientific) for 90 minutes at room temperature, and again prior to application of Pierce ECL Western Blotting Substrate (Thermo Fisher Scientific). Relative band intensity was quantified using the GE Amersham Imager 600 (GE Healthcare Life Sciences) and normalized to GAPDH. Primary antibodies included the following: phospho-Stat5 (\#9314S; RRID: AB_2302702), phospho-Stat3 (\#9138S; RRID: AB_331261), Stat5 (\#9363S; RRID:
AB_2196923), Stat3 (\#9139S; RRID: AB_331757), PPAR $\gamma$ (\#PA3-821A; RRID: AB_2166056), and GAPDH (\#2118S; RRID: AB_561053).

\section{qRT-PCR}

Total RNA was isolated from gastrocnemius muscles of BC-PDOX mice $(n=8)$, NSG control mice $(n=6)$, PDOX-Con mice $(n=5)$, and pectoralis major muscles of patients with breast cancer $(n=20)$, and control female patients $(n=10)$ as described above. Two $\mu \mathrm{g}$ of cDNA was produced using Invitrogen SuperScript III First-Strand Synthesis System (Thermo Fisher Scientific) according to the manufacturer's protocol, and relative expression of selected genes was analyzed using SYBR Green PCR Master Mix (Thermo Fisher Scientific) with the Applied Biosystems 7500 Real-Time PCR System (Thermo Fisher Scientific). Primer efficiencies were determined to be between $90 \%$ and $110 \%$, and relative mRNA expression was calculated using the Pfaffl method (32). qRT-PCR primers (Supplementary Table S1) were designed using Primer3 (33).

\section{Ex vivo muscle physiologic analysis}

Muscle contractile properties were examined in the extensor digitorum longus (EDL) muscles of experimental mice using methods as described previously (34-36). Muscles were transferred to an oxygenated tissue bath containing Ringer solution $\left(100 \mathrm{mmol} / \mathrm{L} \mathrm{NaCl}, 4.7 \mathrm{mmol} / \mathrm{L} \mathrm{KCl}, 3.4 \mathrm{mmol} / \mathrm{L} \mathrm{CaCl}_{2}\right.$, $1.2 \mathrm{mmol} / \mathrm{L} \mathrm{KH}_{2} \mathrm{PO}_{4}, 1.2 \mathrm{mmol} / \mathrm{L} \mathrm{MgSO}_{4}, 25 \mathrm{mmol} / \mathrm{L}$ HEPES, and $5.5 \mathrm{mmol} / \mathrm{L}$-glucose) maintained at $22^{\circ} \mathrm{C}$. Muscle length was adjusted to obtain the maximal twitch response and this length was recorded as optimal length (i.e., Lo). Parameters analyzed from isometric twitch contractions included peak isometric twitch force $(\mathrm{Pt})$, contraction time $(\mathrm{CT}), 1 / 2$ relaxation time $(1 / 2 \mathrm{RT})$, rate of force production, and rate of relaxation. With the muscle set at Lo, the force-frequency relationship was generated to quantify maximal force by stimulating muscles with increasing frequencies as follows: $1,5,10,25,50,80,100,120$, and $150 \mathrm{~Hz}$. Muscle fatigue was analyzed using a repeated stimulation protocol lasting 6 minutes and consisting of repeated $40 \mathrm{~Hz}$ tetanic trains that occurred once every second and lasted $330 \mathrm{~ms}$. LabChart software V7 (AD Instruments, RRID: SCR_001620) was used to obtain the area under the fatigue curve (AUC) and used as a measure of total force produced during the fatigue protocol. To interpolate the fatigue data as a function of time, a piece-wise cubic spline function with 5 evenly spaced interior knots was linearly regressed to the data using the splines (V3.4.0), spline2 (V0.2.8), and stats (V3.4.0) packages in $\mathrm{R}$ (V3.4.0; RRID: SCR_001905). The first derivative of the fit cubic splines and the corresponding 95th percentile confidence intervals were used to determine whether the rate of change in fatigue was significantly different between conditions. Muscle cross-sectional area (CSA) was calculated by dividing the muscle mass by the product of the muscle density coefficient $\left(1.06 \mathrm{~g} \mathrm{~cm}^{3}\right)$, muscle Lo, and the fiber length coefficient (EDL: 0.45). The calculated whole-muscle CSA value was used to calculate specific force (i.e., absolute force $\mathrm{mN}$. muscle $\mathrm{CSA}^{-1}$; refs. 37,38 ).

\section{Statistical analysis}

GraphPad Prism (V5; RRID: SCR_002798) was utilized to analyze the following datasets. Student $t$ test was used $(\alpha=$ $0.05)$ to compare means for protein expression via Western blotting and $\log _{2}$-transformed fold changes $\left[\log _{2}(\mathrm{FC})\right]$ in mRNA 
Wilson et al.

expression in human samples. One-way ANOVA was used to compare $\log _{2}$ (FC) in mRNA expression in murine samples, muscle weights, and selected muscle contractile properties, including AUC, Lo, isometric force, CT, $1 / 2 \mathrm{RT}$, rate of force production, and rate of relaxation. Two-way ANOVA was used to compare differences between groups in force output over time during the 6-minute fatigue protocol. Goodness-of-fit $\left(r^{2}\right)$ for the relationship between STAT3 and STAT5 activation (e.g., pSTAT3/total STAT3) versus each physiologic parameter was analyzed using the linear regression feature of GraphPad Prism, comparing the best-fit linear regression line to $\mathrm{H}_{0}$ where the bestfit line is a horizontal line (i.e., $\mathrm{m}=0$ ) through the mean of all Y values ( $n=8$, pooled NSG and BC-PDOX).

\section{Results}

In silico pathway and transcription factor analysis of RNA-Seq in skeletal muscles from the BC-PDOX mouse model

Muscle tissue from 4 mice bearing unique BC-PDOX tumors representing 4 different patients were used for RNA-Seq (Supplementary Table S2) and compared with muscle tissue from nontumor NSG control mice. Unsupervised clustering analysis identified a unique transcriptional signature present in the BC-PDOX muscle, with a general trend of transcriptional downregulation (Fig. 1B), supporting our previously published transcriptional signature of SkM from early-stage patients with breast cancer (23). Principal component analysis (PCA) shows clustering of the BC-PDOX SkM transcriptional signatures, separate from the more tightly clustered controls (Fig. 1C).

IPA identified several canonical pathways as being dysregulated in this model, with multiple identified pathways having been previously implicated in cancer cachexia and muscle dysfunction in other models, including signaling via PPARs, mTOR, IL1, and IL6. IPA additionally identified several pathways that have not yet been connected to cancer cachexia and therefore warrant further study in the context of tumor-induced SkM dysfunction, such as integrin and gap junctions signaling. Interestingly, IPA predicted no significant dysregulation within 3 pathways that have been strongly implicated in cancer cachexia in other models (Fig. 2A). IPA molecule activity predictor (MAP) predicted inhibition of muscle contraction resulting from aberrant calcium release from the sarcoplasmic reticulum (Fig. 2B). MAP additionally predicted decreased ATP generation in SkM due to decreased activity of electron transport chain complexes IV and V (Fig. 2C).

The observed trend of transcriptional downregulation in both patients with breast cancer (23) and the BC-PDOX SkM transcriptomes suggests the involvement of genome-wide transcriptional reprogramming. We therefore conducted an in silico transcription factor-enrichment analysis of the downregulated transcripts in each model (Fig. 2D), as well as an analysis including all DEGs (Supplementary Fig. S1A and S1B) using Enrichr. This analysis identified PPAR gamma (PPAR $\gamma)$ and nuclear factor (erythroid-derived 2)-like 2 (NFE2L2, also known as NRF2) as transcriptional regulators with altered activity in both models. Consistent with this analysis, NFE2L2/NRF2 and PPAR signaling were also identified as dysregulated by IPA (Fig. 2A). IPA predicted weak inhibition of STAT3 and moderate inhibition of STAT5 signaling in the BC-PDOX SkM relative to NSG control mice (Fig. 2E). Contrary to previous reports on the involvement of STAT3 in tumor-induced SkM dysfunction (39), IPA, Enrichr, or confirmatory Western blotting did not identify significant activation of STAT3 in SkM in our model (Supplementary Fig. S1A-C). Interestingly, we observed a near-significant increase in pSTAT3 and total STAT3, and a statistically significant decrease in total STAT5 (Supplementary Fig. S1D and S1E), but no significant change in the ratio of activated to total STAT protein in either case, suggesting alternative signaling might be involved. In addition, the ratio of activated to total STAT3 or STAT5 protein did not correlate with any muscle contractile parameter obtained (Supplementary Table S3).

Comparison of RNA-Seq profiles in skeletal muscles from human patients with breast cancer and BC-PDOX mice identifies PPAR-signaling as a central regulator of breast tumor-associated transcriptional alterations in muscle tissue

In comparing the SkM-specific gene expression signatures from the BC-PDOX model with the previously published transcriptome of SkM of human patients with breast cancer (23), expression of 40 transcripts were similarly altered, with $85 \%$ of these being downregulated in both models (Fig. 3A). STRING analysis identified a significant functional relationship between the 40 input genes $\left(P=4.87 \mathrm{e}^{-14}\right)$, with PPAR signaling molecules being central to the generated network and insulin signaling molecules as enriched in the 40-gene set (Fig. 3B). Given that 40 genes is a somewhat undersized dataset for enrichment analysis, we utilized 2 additional bioinformatics tools to verify the role of PPAR $\gamma$ as a regulator of mammary tumor-induced SkM alterations. Consistent with our transcription factor analysis of downregulated transcripts in each model (Fig. 2), in silico transcription factor analysis of the 40 overlapping DEGs identified PPAR $\gamma$ as the single significantly enriched transcriptional regulator (adjusted $P=4.8 \mathrm{e}^{-6}$; Fig. 3C). A third gene enrichment tool, GeneAnalytics, also identified PPAR signaling, twice, in the 8 high-scoring pathways enriched in the 40-gene set (Fig. 3D), where high-scoring matches are defined as those with a corrected $P<0.001$ when using a binomial distribution to test the null hypothesis that the 40 input genes are not overrepresented in the pathways noted on the $x$-axis. Negative controls for Enrichr and STRING enrichment analyses using a random set of 40 protein-coding genes identified no significant enrichments, with all adjusted $P>0.9$ (Supplementary Fig. S1F and S1G).

Verification and validation of RNA-Seq profiles in skeletal muscles from BC-PDOX mice and human patients with breast cancer

The gene expression of Pparg and a subset of known and predicted Pparg targets identified in Fig. 3A (Cidec, Fabp4, Rbp4, Slc1a5) were verified and validated by qRT-PCR in the same samples used in RNA-Seq as well as in a new cohort of SkM samples, respectively. In muscles from the original cohort of BC-PDOX mice, the median $\log _{2}$ (FC) was negative for all genes, indicating downregulation of gene expression. In the verification samples, Cidec, Pparg, Rbp4, and Slc1a5 were significantly downregulated. In the validation samples, Fabp4 was significantly downregulated, and Cidec and Pparg showed a statistical trend for downregulation (Fig. 4A). In muscles from human patients with breast cancer, the median $\log _{2}$ (FC) was negative for all samples, indicating downregulation of gene expression. In the verification samples, Fabp4, Pparg, Rbp4, and Slc1a5 were significantly downregulated, whereas Cidec showed a statistical trend for downregulation. In the validation samples, 
A

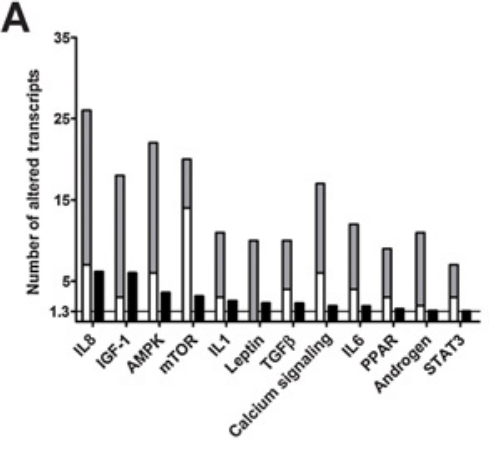

C
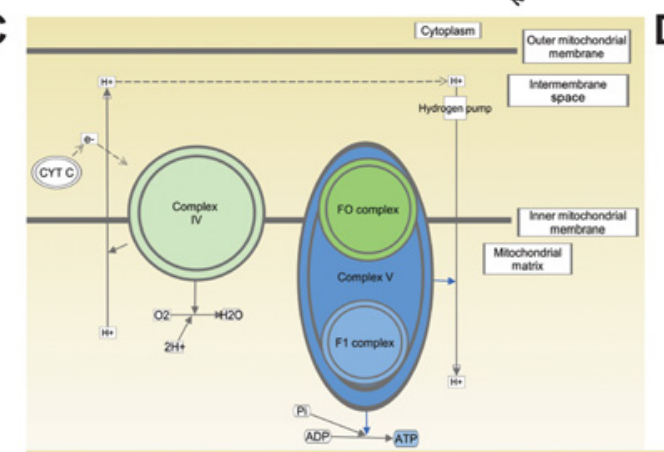

B

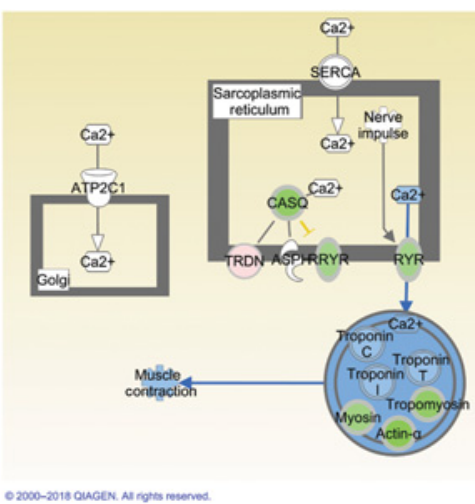

E
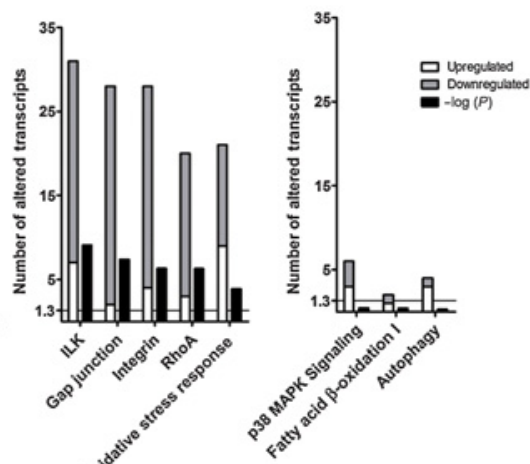

BC Patients, input: downregulated genes
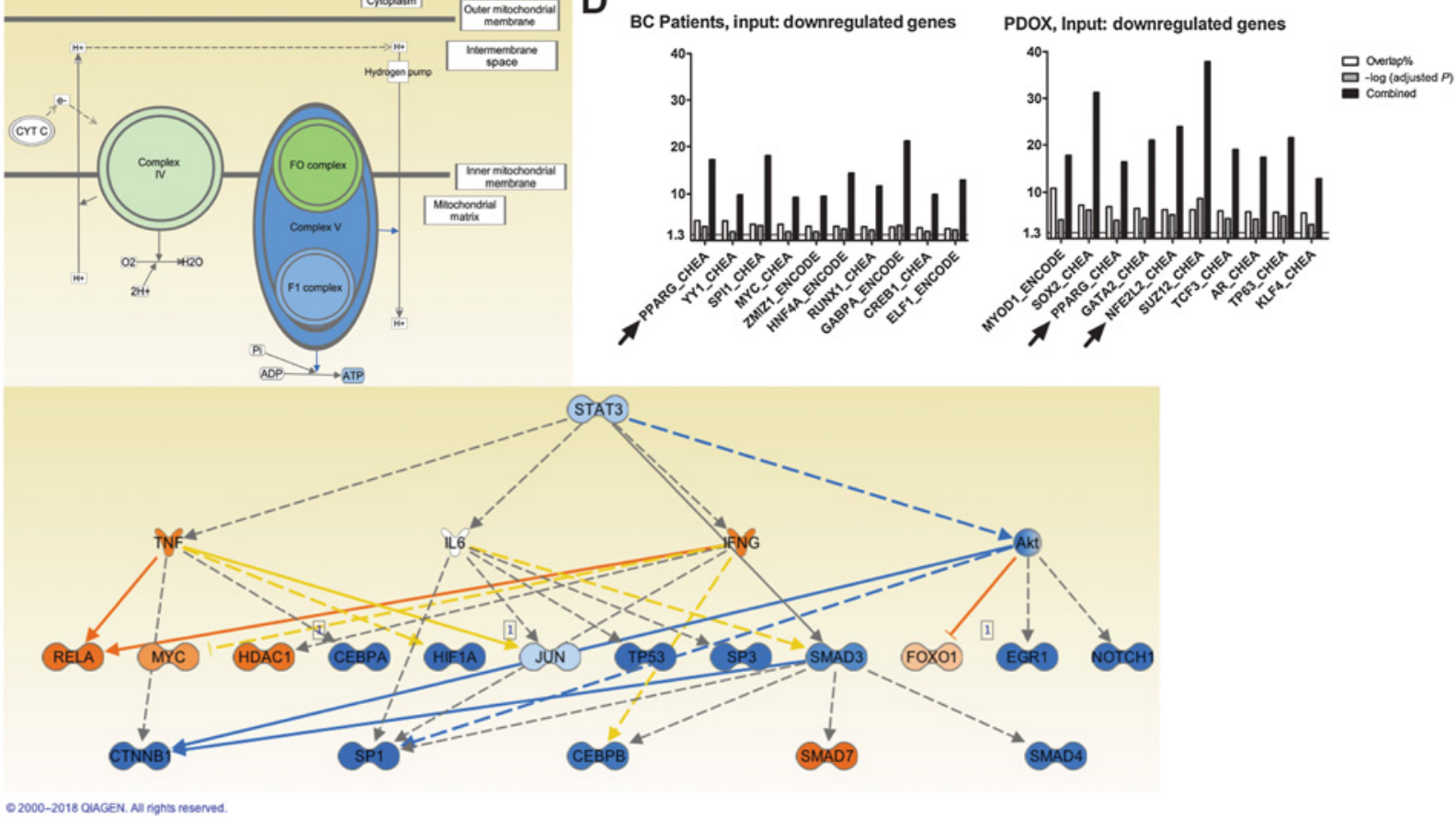

Figure 2.

In silico pathway and transcription factor analysis of RNA-Seq data. A, Selected canonical pathways identified by IPA in the transcriptome of BC-PDOX mice. For each pathway listed on the $x$-axis, the $y$-axis reports the raw number of differentially expressed genes in the BC-PDOX SkM relative to NSG controls, separated into up- and downregulated transcripts. The $y$-axis additionally reports the $-\log (P)$ reported by IPA; $-\log (P)>1.33$ is equivalent to $P<0.05$. The figure reports pathways identified by IPA as significantly dysregulated in our model that have been previously implicated in cancer cachexia (left), significantly dysregulated in our model but not previously implicated in cancer cachexia (center), or previously implicated in cancer cachexia but not identified by IPA as significantly dysregulated in our model (right). B, IPA predicts decreased release of calcium from the sarcoplasmic reticulum in the SkM of BC-PDOX mice resulting in decreased muscle contraction. C, Complex IV and V of the electron transport chain are predicted by IPA to have decreased activity in the BC-PDOX mice relative to NSG controls, resulting in decreased ATP production. D, Top ten most highly ranked transcriptional regulators identified by Enrichr as regulating the transcriptional alterations observed in SkM of BC-PDOX and human patients with breast cancer where the input gene list was exclusively downregulated transcripts. Transcription factors are ranked by overlap\% (calculated as [(transcripts regulated by transcription factor $x$ ) $\cap$ (transcripts differentially expressed in SkM of tumor-bearing subject)]/(transcripts regulated by transcription factor $x$ ). The $y$-axis reports overlap\%, -log (adjusted $P$ ), and combined scores for each transcription factor as reported by Enrichr. Arrows identify PPARG and NFE2L2/NRF2. E, IPA upstream regulator analytic predicts weak inhibition of STAT3. Key for (B, C, E): green shading indicates decreased expression of transcripts coding for the shaded entity, red shading indicates increased expression of transcripts coding for the shaded entity, blue shading indicates decreased predicted activity/production of the shaded entity, and orange shading indicates increased predicted activity/production of the shaded entity.

Pparg and Slc1a5 were significantly downregulated, whereas Cidec and Fabp4 showed a statistical trend for downregulation (Fig. 4B). The protein abundance of PPAR $\gamma$ was evaluated in muscles from patients with breast cancer. Although there were no differences in total PPAR $\gamma$ protein between control and patients with breast cancer, we did observe a greater abundance of a truncated form of $\operatorname{PPAR} \gamma$, at approximately $40 \mathrm{kDa}$, in the muscles from patients with breast cancer (Fig. 4C). Following orthotopic implantation of the syngeneic mouse breast tumor cell line, E0771, Pparg gene expression in SkM was lower in weight-stable tumor-bearing mice 
Wilson et al.

A

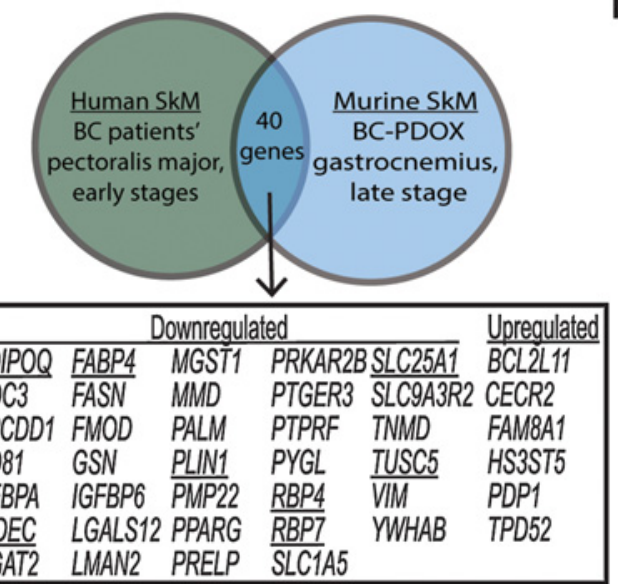

B

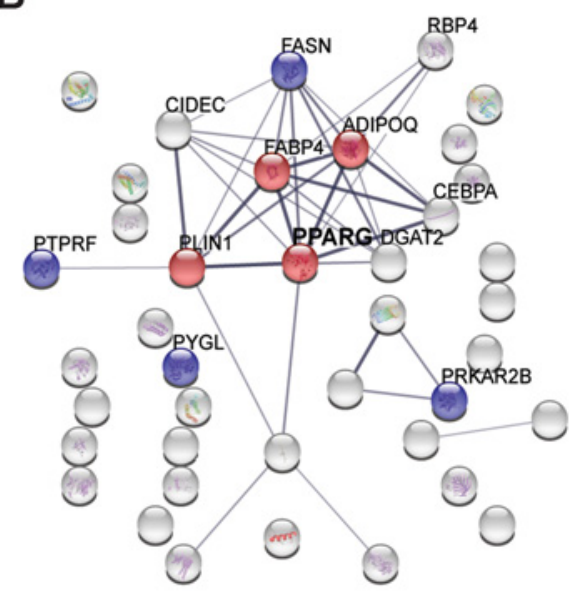

C
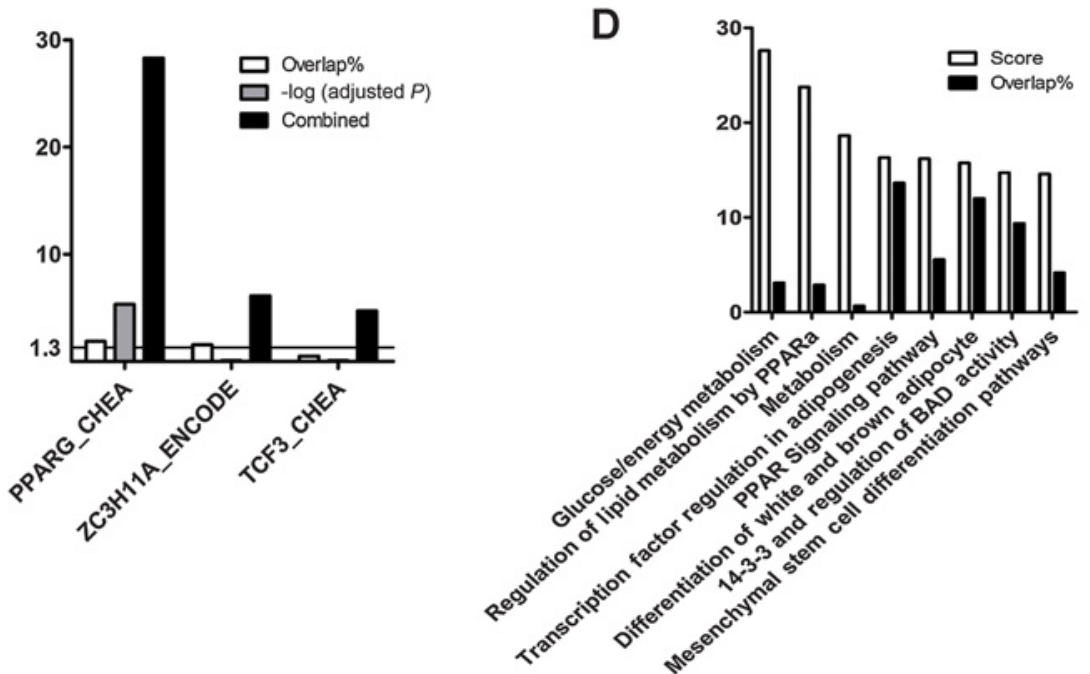

Figure 3.

Genes with altered expression in patients with breast cancer and BC-PDOX mice identifies PPAR signaling as potential driver of breast cancer-induced SkM Fatigue. A, Venn diagram identifying 40 genes with commonly altered expression (e.g., downregulated in both sets) in both human patients with breast cancer and BC-PDOX mice, with experimentally verified targets of PPARs underlined. B, STRING in silico protein-protein interaction analysis identifies significant functional interactions between the 40 commonly altered transcripts ( $P=4.87 \mathrm{e}-14)$, with PPAR $\gamma$ and related signaling molecules central to the generated network of protein-protein interactions present in the set of 40 commonly altered transcripts (red, KEGG 03320, FDR $=0.003$ ). Insulin signaling molecules are also significantly enriched in this gene set (blue, KEGG 04910, FDR =0.018). C, PPAR signaling is identified by GeneAnalytics twice in the eight pathways identified as high-scoring matches (corrected $P<0.0001$ ). D, Enrichr in silico transcription factor analysis (ENCODE and ChEA Consensus from ChIP-X) identified PPAR $\gamma$ as the single significantly enriched transcriptional regulator in the 40 commonly altered transcripts. The $y$-axis reports overlap\%, -log (adjusted $P$ ), and combined scores for each transcription factor as reported by Enrichr; $-\log ($ adjusted $P$ ) $>1.33$ is equivalent to adjusted $P<0.05$.

and was greater in tumor-bearing mice that lost body weight (Supplementary Fig. S2).

Tumor growth in BC-PDOX mice induces a greater rate of skeletal muscle fatigue and a slowing of isometric contractile properties

EDL muscles from BC-PDOX-bearing mice, NSG control mice, and PDOX-Con mice were stimulated ex vivo using a repeated contraction protocol to analyze muscle fatigue properties in response to $\mathrm{BC}-\mathrm{PDOX}$ growth. The shape of the fatigue index curve from muscles of BC-PDOX mice was significantly different than the curve from NSG control mice and PDOX-Con mice, especially during the first 80 s of the protocol, with force output declining rapidly from the initiation of the protocol (Fig. 5A) AUC was also significantly lower in muscles from BC-PDOX mice compared with muscles from NSG control mice and showed a statistical trend when compared with muscles from PDOX-Con mice (Fig. 5B). These fatigue responses in mice implanted with $\mathrm{BC}$-PDOXs are strikingly similar to the muscle fatigue responses we previously reported in an orthotopic syngeneic breast cancer model using C57BL/6 mice (23). Fatigue data were also interpolated as a function of time to evaluate the rate of change in muscle fatigue during different phases of the fatigue protocol (Fig. 5C). The rate of change was significantly greater in muscles from BC-PDOX mice compared with muscles from both control groups during the early phase of the fatigue protocol (i.e., first 
A

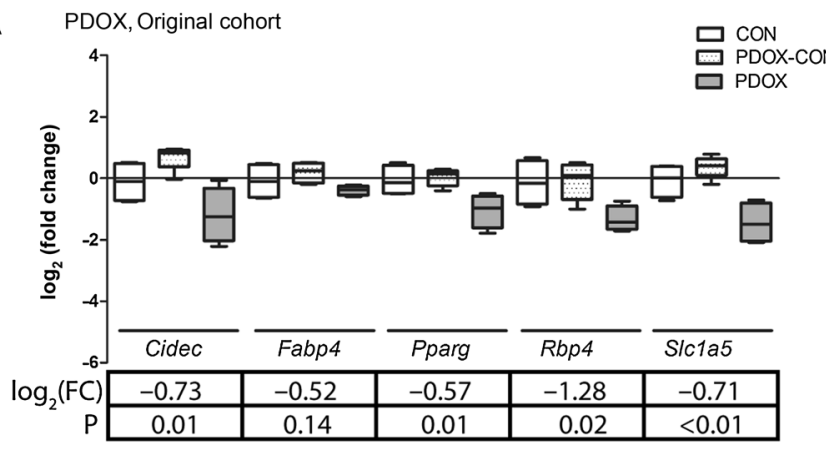

B

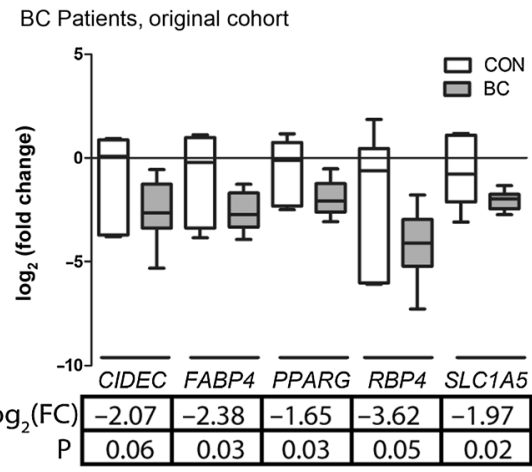

BC Patients, new cohort

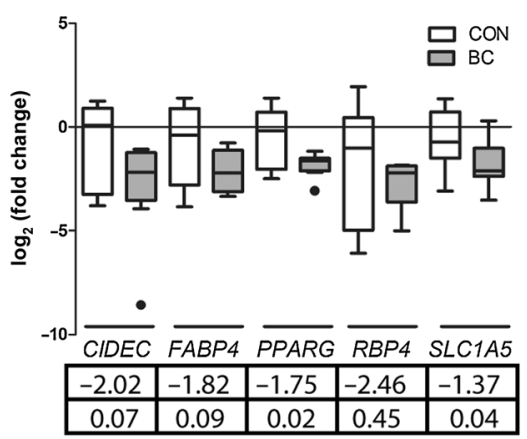

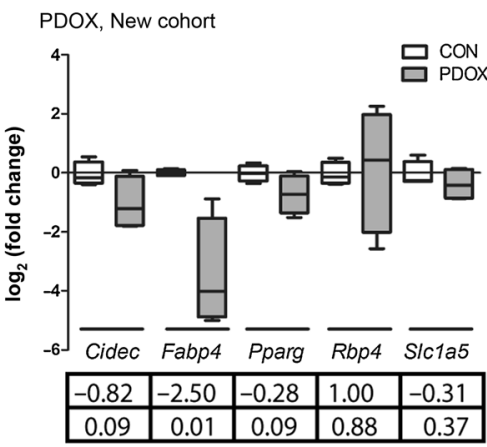

C

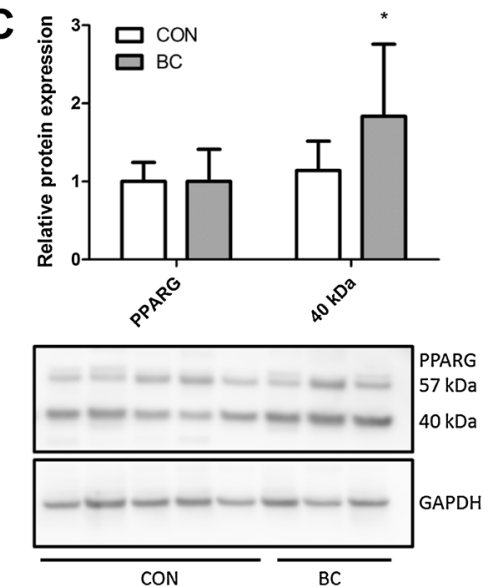

Figure 4.

qRT-PCR and protein verification and validation of RNA-Seq. QRT-PCR was performed for Pparg and a subset of known and predicted Pparg target genes to both verify and validate the RNA-Seq completed in muscles from BC-PDOX mice and human patients with breast cancer. A, Verification and validation of RNA-Seq in muscle samples from control and BC-PDOX mice. B, Verification and validation of RNA-Seq in muscle samples from control and patients with breast cancer. Each graph includes the $\log _{2}$ (FC) and associated $P$ value for the gene analyzed. C, Protein quantification of PPAR $\gamma$ in muscle samples from control $(n=5)$ and patients with breast cancer $(n=15)$. One representative blot is presented. ${ }^{*}, P<0.05$.

150 contractions). Thus, muscles from tumor-bearing mice have a greater rate of fatigue and reach their threshold force values earlier in the fatigue protocol. Similar rate of change curves were obtained in EDL muscles from immunocompetent C57BL/ 6 mice following 4 weeks of orthotopic E0771 mammary tumor growth (Supplementary Fig. S3A). These data demonstrate a greater rate of muscle fatigue in response to mammary tumor growth regardless of the host's immune status, providing strong support for an effect of mammary tumor growth on inducing muscle fatigue.

Isometric contractile data for the EDL muscles in all groups are presented in Table 1. Although there was no significant difference in $\mathrm{CT}$, the rate of force development of twitch contractions was lower in muscles from BC-PDOX mice. In addition, $1 / 2$ RT was longer and the rate of relaxation was lower in EDL muscles from BC-PDOX mice. Similar contractile properties were observed in EDL muscles from mice bearing syngeneic E0771 mammary tumors (Supplementary Fig. S3B). Absolute twitch and tetanic forces were lower in muscles from BC-PDOX mice. However, specific twitch and tetanic forces were not different between groups. There were no significant differences in the absolute mass of EDL, soleus, gastrocnemius, or tibialis anterior muscles among the groups (Fig. 5D). Collectively, these data indicate that isometric twitch properties of the fast EDL muscle are slowed in response to mammary tumor growth in the absence of quantifiable differences in muscle mass and normalized isometric force output.

\section{Discussion}

This study tested the hypothesis that the BC-PDOX mouse model would recapitulate the common clinical phenomenon of breast cancer-induced SkM fatigue in the absence of muscle wasting. We found that BC-PDOX-bearing mice indeed show greater SkM fatigability than nontumor-bearing NSG control mice and PDOX-Con mice, despite no differences in absolute muscle mass. We then discovered that SkM from BC-PDOXbearing mice exhibits widespread transcriptional changes, including alterations in pathways previously reported to be relevant to cancer cachexia (e.g., PPAR, mTOR, IL6, IL1) as well as those that have never been reported in this condition (e.g., signaling via integrins and gap junctions). These data validate the use of BC-PDOX as a model of breast cancer-induced SkM dysfunction and identify novel therapeutic targets to improve muscle function in patients with breast cancer.

We have recently published the unique transcriptional signature of SkM from patients with early-stage nonmetastatic breast cancer, which showed transcriptional alterations in pathways involved in mitochondrial function, oxidative phosphorylation, 
Wilson et al.

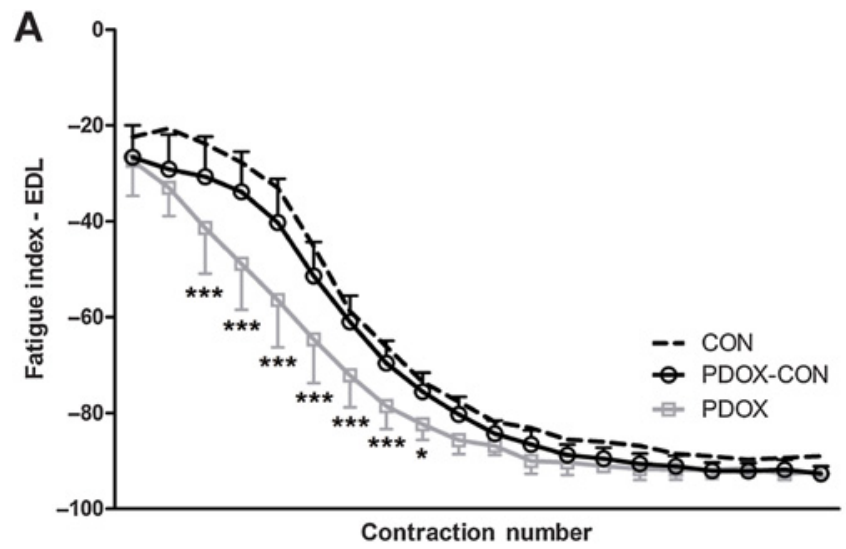

B

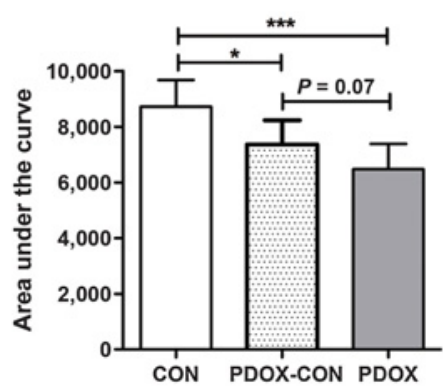

C

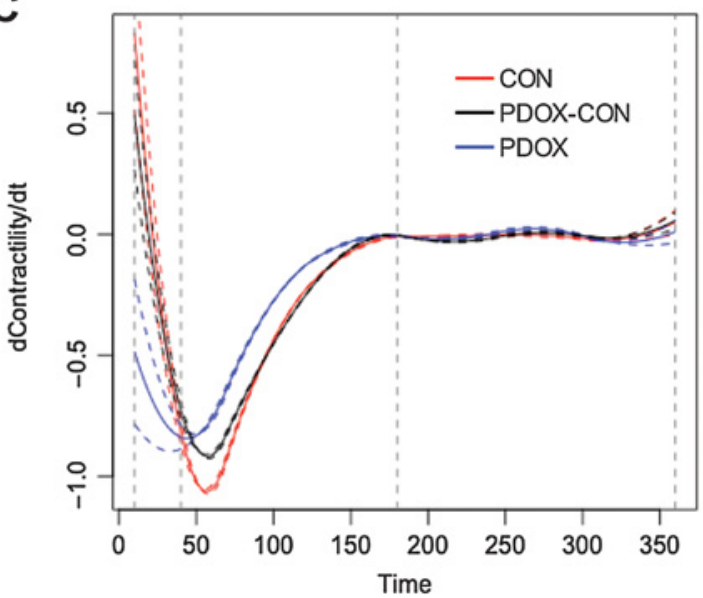

D

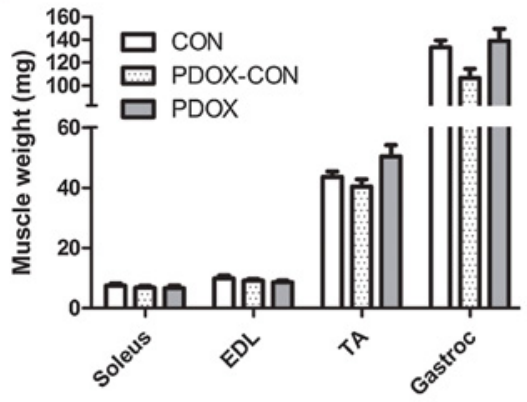

Figure 5 .

BC-PDOX induces SkM fatigue. A, Average ex vivo SkM fatigue curves generated for NSG control animals (NSG, $n=6$ ), surgical control animals (PDOX-CON, $n=5)$, and BC-PDOX mice $(n=6)$, using the EDL muscle. The leftward shift of the BC-PDOX fatigue curve indicates greater fatigability. The chart presents mean fatigue index \pm SEM for every 10th contraction in the fatigue protocol; PDOX-CON versus PDOX groups compared via 2-way ANOVA. B, AUC for the fatigue curves presented in A. C, First-derivative curves generated for the fatigue curves presented in A, representing the rate of change in force output of the EDL muscle. D, Absolute mass comparisons of EDL, soleus, gastrocnemius, and tibialis anterior muscles in the BC-PDOX mice, NSG mice, and PDOX-CON, showing no significant differences; ${ }^{*}, P<0.05 ;{ }^{* *}, P<0.01$, $^{* *}, P<0.001$; means compared via 1-way ANOVA unless otherwise stated.

and PPAR signaling (23). These data revealed that SkM responds to breast tumor growth with a general downregulation of transcriptional activity; a response that was not affected by breast tumor subtype or whether patients received chemotherapy prior to surgery and biopsy acquisition. In addition, the gene networks identified were dysregulated in SkM at a time when cachexia (i.e., muscle wasting) was likely not evident, as both body mass index and serum albumin levels were not different between patients with breast cancer and patients without breast cancer in this study (23). In this study, we extend these observations by analyzing a cohort of immunocompromised mice bearing human breast cancer tumors of multiple molecular subtypes, including luminal, triple-negative, and HER2-overexpressing tumors. When comparing the transcriptional signature of SkM in human patients with breast cancer with SkM from BC-PDOX mice, similar patterns emerged with respect to affected markers and pathways, with

Table 1. Ex vivo isometric contractile parameters in the EDL muscle

\begin{tabular}{|c|c|c|c|c|}
\hline & NSG & BC-PDOX & PDOX-Con & $\boldsymbol{P}$ \\
\hline$\overline{E D L}$ Lo $(\mathrm{mm})$ & $11.5 \pm 0.4$ & $11.8 \pm 0.4$ & $11.1 \pm 1.1$ & 0.0985 \\
\hline Twitch $(\mathrm{mN})$ & $38.5 \pm 4.4$ & $33.3 \pm 7.9$ & $51.9 \pm 7.1$ & 0.0001 \\
\hline Twitch $\left(\mathrm{mN} \cdot \mathrm{CSA}^{-1}\right)$ & $40.4 \pm 19.6$ & $34.4 \pm 8.1$ & $44.6 \pm 8.1$ & 0.3178 \\
\hline Contraction time (ms) & $21.4 \pm 6.9$ & $25.0 \pm 5.5$ & $23.0 \pm 9.5$ & 0.7220 \\
\hline $1 / 2$ Relaxation time (ms) & $31.7 \pm 4.1$ & $46.0 \pm 5.5$ & $33.3 \pm 7.1$ & 0.0017 \\
\hline Rate of force development $\left(\mathrm{mN} \cdot \mathrm{s}^{-1}\right)$ & $1,481 \pm 339.3$ & $1,038 \pm 180.1$ & $1,867 \pm 415.2$ & 0.0010 \\
\hline Rate of relaxation $\left(\mathrm{mN} \cdot \mathrm{s}^{-1}\right)$ & $-682.3 \pm 46.2$ & $-450.9 \pm 174.2$ & $-921.5 \pm 169.5$ & 0.0001 \\
\hline Tetanus (mN) & $201.1 \pm 22.5$ & $179.6 \pm 41.3$ & $243.7 \pm 28.0$ & 0.0015 \\
\hline Tetanus $\left(\mathrm{mN} \cdot \mathrm{CSA}^{-1}\right)$ & $204.3 \pm 85.2$ & $184.6 \pm 40.9$ & $212.8 \pm 33.3$ & 0.6233 \\
\hline
\end{tabular}


40 genes concordantly affected by breast tumor growth in SkM from human patients with breast cancer and mice bearing human breast tumors. This set of 40 genes represents those that are dysregulated early in the course of breast cancer progression (patients with breast cancer), remain dysregulated into the late stages of disease (BC-PDOX model), and are altered in 2 different SkMs (pectoralis major vs. gastrocnemius). Moreover, our in silico analysis strongly implicates PPAR signaling in the transcriptional alterations observed in SkM in response to tumor growth, and a large percentage of the DEGs common to both BC-PDOX and breast cancer patients' SkM are either verified or predicted targets of PPARs. A subset of these PPAR targets, including Cidec, Fabp4, $R b p 4$, and Slc1a5 are central to the network of protein-protein interactions present in the SkM of both patients with breast cancer and BC-PDOX mice and were validated and verified in both mouse and human muscle samples. Therefore, it is probable that these genes and their associated networks represent a significant driving force behind breast cancer-induced muscle dysfunction and fatigue and further suggest that PPAR signaling may be mechanistically central to breast cancer-associated SkM dysfunction. Although Pparg gene expression was downregulated in muscles of human patients with breast cancer, we did not observe a concomitant reduction in PPAR $\gamma$ protein abundance. However, our PPAR $\gamma$ antibody did recognize a lower molecular weight $(\sim 40 \mathrm{kDa})$ protein with a greater abundance in muscles from patients with breast cancer. Studies have suggested that truncated forms of PPAR $\gamma$, which are cleaved by active caspases, decrease the transcriptional activity of PPAR $\gamma$ by not translocating to the nucleus (40-42). This mechanism of action is consistent with our analyses, in which multiple downstream targets of PPAR $\gamma$ are transcriptionally downregulated in muscles of patients with breast cancer and BC-PDOX mice.

PPARs are lipid-sensing, ligand-activated transcription factors that regulate multiple metabolic processes throughout the body, with a particularly strong role in regulation of glucose and lipid metabolism. PPAR $\alpha$ is predominantly expressed in tissues with high rates of fatty acid catabolism, including liver, SkM, and cardiac muscle. PPAR $\gamma$ is highly expressed in adipose tissue, is considered a master regulator of adipogenesis, and modulates insulin sensitivity. PPAR $\beta / \delta$ is the least well-characterized of the PPARs, but it is ubiquitously expressed in most tissues, regulates blood cholesterol and glucose levels, and is involved in fatty acid oxidation (43). Because PPARs play an obvious role in regulating whole body energy homeostasis, there has been significant success in modulating these transcription factors for the benefit of patients with diabetes, dyslipidemia, and atherosclerosis (44); and promising anticatabolic efficacy has been observed with PPAR agonists in mouse models of Lewis lung carcinoma (45), colon adenocarcinoma (46), and non-small-cell lung cancer (47). PPAR $\gamma$ is 1 of the main regulators of adipocyte differentiation $(48,49)$ and is downregulated in SkM of patients with breast cancer (23) and BC-PDOX-bearing mice. Clinical trials utilizing PPAR $\gamma$ agonists have been undertaken for breast cancer previously, as there was a hypothesis that the agonists would cause the mammary tumor cells to undergo terminal differentiation and cease proliferation $(50,51)$. A phase II trial using the oral antidiabetic drug and PPAR $\gamma$ ligand troglitazone in patients with advanced breast cancer showed no benefit in terms of breast cancer progression, but this study did not report assessments of body weight, muscle strength, or muscle fatigue. The drug was tolerated well by patients (52), however. As our data indicate that downregulation of PPAR $\gamma$ transcriptional activity may play an important role in mediating breast cancer-associated muscle dysfunction, further studies assessing the efficacy of PPAR $\gamma$ agonists in ameliorating breast cancer-induced fatigue are warranted.

BC-PDOX tumor growth in female mice resulted in increased SkM fatigability, decreased rate of force development, decreased rate of twitch relaxation, and increased $1 / 2$ RT in the fast EDL muscle. This shift to a slower, more fatigable EDL occurred despite no change in EDL mass relative to control mice. The rate of twitch force development and relaxation are both strongly influenced by calcium release and reuptake in specialized compartments of the myocyte. The rate of force development in SkM depends upon the rate of calcium release from the sarcoplasmic reticulum (SR) through the ryanodine receptors (RyR), and the rate of SkM relaxation is strongly influenced by the removal of calcium ions from the cytoplasm by the sarco/endoplasmic reticulum $\mathrm{Ca}^{2+}$ ATPase (SERCA; refs. 53, 54). Expression of multiple key regulators of calcium flow in SkM were altered, and IPA identified canonical calcium signaling pathways as being significantly altered in the BC-PDOX SkM. Expression of transcripts encoding calsequestrin 1 (casq1), calsequestrin 2 (casq2), and RyR were all decreased, and IPA predicted impaired calcium release from the $\mathrm{SR}$ as a direct result of these transcriptional alterations, which may explain the slowed rate of force development in BC-PDOX SkM. However, we did not observe a change in mRNA expression of any SERCA isoform (adjusted $P$ values of $0.64,0.87$, and 0.92 for Atp2a1, Atp2a2, and Atp2a3, respectively). Our data directly conflict with recent reports describing increased expression of calsequestrin (39) and SERCA isoforms $(39,55)$ in SkM of tumorbearing mice. This discrepancy could reflect differences in the animal models used, cancer types studied, or stage of cancer cachexia progression.

A recent report quantifying SkM deficits in male $\mathrm{APC}^{\mathrm{Min} /+}$ mice during cachexia progression similarly identified a slower, more fatigable phenotype in the SkM of weight-stable mice, with STAT3 activation correlating with alterations in the rate of twitch contraction and relaxation (39). In our BC-PDOX model, we observed alterations in the rate of twitch contraction and relaxation in the absence of STAT3 activation, and STAT3 activation did not correlate with any muscle contractile parameter obtained from the BC-PDOX mice. This discrepancy indicates that while inflammation correlates with the slowing of SkM physiology in 1 model, inflammatory signaling via STAT3 is likely not associated with the slowed muscle contractile parameters observed in BC-PDOX mice.

Collectively, our data show that breast cancer-induced muscle dysfunction can be effectively recapitulated and studied in a $\mathrm{BC}-\mathrm{PDOX}$ model. The overall slowing and greater rate of fatigue of the fast EDL muscle, coupled with altered expression of several key regulators of SkM calcium handling, suggest that alterations in calcium handling contribute to the slow, fatigable phenotype induced by tumor growth. In addition, 3 independent in silico analyses identified PPAR $\gamma$ signaling as highly dysregulated in both BC-PDOX SkM and in the muscle of patients with early-stage breast cancer, suggesting that PPAR agonists may be of clinical benefit to patients with breast cancer experiencing fatigue. In conclusion, PPAR signaling, particularly PPAR $\gamma$ signaling, likely mediates a significant portion of mammary tumor's impact on SkM. Further studies are warranted to determine whether PPAR agonists would provide a quality-of-life benefit to patients with breast cancer with muscle dysfunction. Given that the side effect 
Wilson et al.

profile of these drugs is relatively benign, PPAR agonists may prove useful as an additive therapy to alleviate cancer-induced muscle fatigue.

\section{Disclosure of Potential Conflicts of Interest}

No potential conflicts of interest were disclosed.

\section{Disclaimer}

The content is solely the responsibility of the authors and does not necessarily represent the official views of the NIH.

\section{Authors' Contributions}

Conception and design: E.E. Pistilli

Development of methodology: E.N. Pugacheva, E.E. Pistilli

Acquisition of data (provided animals, acquired and managed patients, provided facilities, etc.): H.E. Wilson, K.K. Rhodes, I. Chahal, D.A. Stanton, J. Bohlen, H. Hazard-Jenkins, E.N. Pugacheva, E.E. Pistilli

Analysis and interpretation of data (e.g., statistical analysis, biostatistics, computational analysis): H.E. Wilson, D. Rodriguez, I. Chahal, D.A. Stanton, J. Bohlen, M. Davis, A.M. Infante, D.J. Klinke, E.E. Pistilli

Writing, review, and/or revision of the manuscript: H.E. Wilson, I. Chahal,

J. Bohlen, M. Davis, D.J. Klinke, E.N. Pugacheva, E.E. Pistilli

\section{References}

1. Katz AM, Katz PB. Diseases of the heart in the works of Hippocrates. Br Heart J 1962;24:257-64.

2. Warren S. The immediate causes of death in cancer. The Am J Med Sci 1932; 184:610-5.

3. Aoyagi T, Terracina KP, Raza A, Matsubara H, Takabe K. Cancer cachexia, mechanism and treatment. World J Gastrointest Oncol 2015;7:17-29.

4. Evans WJ, Morley JE, Argiles J, Bales C, Baracos V, Guttridge D, et al. Cachexia: a new definition. Clin Nutr 2008;27:793-9.

5. Fearon K, Strasser F, Anker SD, Bosaeus I, Bruera E, Fainsinger RL, et al Definition and classification of cancer cachexia: an international consensus. Lancet Oncol 2011;12:489-95.

6. Yavuzsen T, Davis MP, Walsh D, LeGrand S, Lagman R. Systematic review of the treatment of cancer-associated anorexia and weight loss. J Clin Oncol 2005;23:8500-11.

7. Fearon K, Arends J, Baracos V. Understanding the mechanisms and treatment options in cancer cachexia. Nat Rev Clin Oncol 2013;10:90-9.

8. Argilés JM, Busquets S, Stemmler B, López-Soriano FJ. Cancer cachexia: understanding the molecular basis. Nat Rev Cancer 2014;14:754-62.

9. Aversa Z, Costelli P, Muscaritoli M. Cancer-induced muscle wasting: latest findings in prevention and treatment. Ther Adv Med Oncol 2017;9: 369-82.

10. Madeddu C, Mantovani G, Gramignano G, Macciò A. Advances in pharmacologic strategies for cancer cachexia. Expert Opin Pharmacother 2015; $16: 2163-77$.

11. Blesch KS, Paice JA, Wickham R, Harte N, Schnoor DK, Purl S, et al Correlates of fatigue in people with breast or lung cancer. Oncol Nurs Forum 1991;18:81-7.

12. Bower JE, Ganz PA, Desmond KA, Rowland JH, Meyerowitz BE, Belin TR. Fatigue in breast cancer survivors: occurrence, correlates, and impact on quality of life. J Clin Oncol 2000;18:743-53.

13. Curt GA, Breitbart W, Cella D, Groopman JE, Horning SJ, Itri LM, et al. Impact of cancer-related fatigue on the lives of patients: new findings from the fatigue coalition. Oncologist 2000;5:353-60.

14. Evans WJ, Lambert CP. Physiological basis of fatigue. Am J Phys Med Rehabil 2007;86:S29-46

15. Fox KM, Brooks JM, Gandra SR, Markus R, Chiou CF. Estimation of cachexia among cancer patients based on four definitions. J Oncol 2009;2009:693458.

16. Kilgour RD, Vigano A, Trutschnigg B, Hornby L, Lucar E, Bacon SL, et al. Cancer-related fatigue: the impact of skeletal muscle mass and strength in patients with advanced cancer. J Cachexia Sarcopenia Muscle 2010;1: $177-85$.
Administrative, technical, or material support (i.e., reporting or organizing data, constructing databases): H.E. Wilson, A.M. Infante, E.E. Pistilli Study supervision: E.E. Pistilli

\section{Acknowledgments}

The authors thank Linda Metheny-Barlow, PhD, for providing the E0771 tumor cells for this project. This research was supported by grants from the National Institute Of General Medical Sciences of the NIH under Award Number P20GM121322, American Cancer Society Institutional Research Grant (09-061-04, to E.E. Pistilli), and the WVCTSI through the National Institute of General Medical Sciences (U54GM104942, to S. Hodder). Additional support for this research was provided by WV-INBRE (P20GM103434), (CA193473, to D.J. Klinke), and (CA148671, to E.N. Pugacheva) from the NIH/NCI in part by a NIH/NCRR5 P20-RR016440-09. The WVU HSC Core Facilities were supported by the NIH grants P30- RR032138/GM103488, S10-RR026378, S10-RR020866, S10-OD016165, and P20GM103434.

The costs of publication of this article were defrayed in part by the payment of page charges. This article must therefore be hereby marked advertisement in accordance with 18 U.S.C. Section 1734 solely to indicate this fact.

Received May 18, 2018; revised October 19, 2018; accepted December 12 2018; published first December 17, 2018.

17. Stephens NA, Gray C, MacDonald AJ, Tan BH, Gallagher IJ, Skipworth RJ, et al. Sexual dimorphism modulates the impact of cancer cachexia on lower limb muscle mass and function. Clin Nutr 2012;31: 499-505.

18. Neefjes ECW, van den Hurk RM, Blauwhoff-Buskermolen S, van der Vorst MJDL, Becker-Commissaris A, de van der Schueren MAE, et al. Muscle mass as a target to reduce fatigue in patients with advanced cancer. J Cachexia Sarcopenia Muscle 2017;8:623-9.

19. Baracos VE, Reiman T, Mourtzakis M, Gioulbasanis I, Antoun S. Body composition in patients with non-small cell lung cancer: a contemporary view of cancer cachexia with the use of computed tomography image analysis. Am J Clin Nutr 2010;91:1133S-7S.

20. Wallengren O, Iresjö BM, Lundholm K, Bosaeus I. Loss of muscle mass in the end of life in patients with advanced cancer. Support Care Cancer 2015; 23:79-86.

21. Anderson LJ, Liu H, Garcia JM. Sex differences in muscle wasting. Adv Exp Med Biol 2017;1043:153-97.

22. Hoffman RM. Patient-derived orthotopic xenografts: better mimic of metastasis than subcutaneous xenografts. Nat Rev Cancer 2015;15:451-2.

23. Bohlen J, McLaughlin SL, Hazard-Jenkins H, Infante AM, Montgomery C, Davis $M$, et al. Dysregulation of metabolic-associated pathways in muscle of breast cancer patients: preclinical evaluation of interleukin-15 targeting fatigue. J Cachexia Sarcopenia Muscle 2018;9:701-14.

24. DeRose YS, Gligorich KM, Wang G, Georgelas A, Bowman P, Courdy SJ, et al. Patient-derived models of human breast cancer: protocols for in vitro and in vivo applications in tumor biology and translational medicine. Curr Protoc Pharmacol 2013; Chapter 14:Unit14.23.

25. Dunphy KA, Tao L, Jerry DJ. Mammary epithelial transplant procedure. J Vis Exp 2010 Jun 10;(40). pii: 1849. doi: 10.3791/1849.

26. Pistilli EE, Jackson JR, Alway SE. Death receptor-associated pro-apoptotic signaling in aged skeletal muscle. Apoptosis 2006;11:2115-26.

27. Patro R, Duggal G, Love MI, Irizarry RA, Kingsford C. Salmon provides fast and bias-aware quantification of transcript expression. Nat Methods 2017; 14:417-9.

28. Soneson C, Love MI, Robinson MD. Differential analyses for RNA-seq: transcript-level estimates improve gene-level inferences. F1000 Res 2015;4: 1521.

29. Szklarczyk D, Morris JH, Cook H, Kuhn M, Wyder S, Simonovic M, et al. The STRING database in 2017: quality-controlled protein-protein association networks, made broadly accessible. Nucleic Acids Res 2017;45:D362-D8.

30. Kuleshov MV, Jones MR, Rouillard AD, Fernandez NF, Duan Q, Wang Z, et al. Enrichr: a comprehensive gene set enrichment analysis web server 2016 update. Nucleic Acids Res 2016;44:W90-7. 
31. Ben-Ari Fuchs S, Lieder I, Stelzer G, Mazor Y, Buzhor E, Kaplan S, et al. GeneAnalytics: an integrative gene set analysis tool for next generation sequencing, RNAseq and microarray data. OMICS 2016;20:139-51.

32. Pfaffl MW. A new mathematical model for relative quantification in realtime RT-PCR. Nucleic Acids Res 2001;29:e45.

33. Untergasser A, Cutcutache I, Koressaar T, Ye J, Faircloth BC, Remm M, et al. Primer3-new capabilities and interfaces. Nucleic Acids Res 2012; 40:e115.

34. O'Connell G, Guo G, Stricker J, Quinn LS, Ma A, Pistilli EE. Muscle-specific deletion of exons 2 and 3 of the IL15RA gene in mice: effects on contractile properties of fast and slow muscles. J Appl Physiol 2015;118:437-48.

35. Pistilli EE, Alway SE, Hollander JM, Wimsatt JH. Aging alters contractile properties and fiber morphology in pigeon skeletal muscle. J Comp Physiol B 2014;184:1031-9.

36. Pistilli EE, Bogdanovich S, Garton F, Yang N, Gulbin JP, Conner JD, et al. Loss of IL-15 receptor $\alpha$ alters the endurance, fatigability, and metabolic characteristics of mouse fast skeletal muscles. J Clin Invest 2011;121: 3120-32.

37. Brooks SV, Faulkner JA. Contractile properties of skeletal muscles from young, adult and aged mice. J Physiol 1988;404:71-82.

38. Lynch GS, Hinkle RT, Chamberlain JS, Brooks SV, Faulkner JA. Force and power output of fast and slow skeletal muscles from mdx mice 6-28 months old. J Physiol 2001;535:591-600.

39. VanderVeen BN, Hardee JP, Fix DK, Carson JA. Skeletal muscle function during the progression of cancer cachexia in the male ApcMin/+ mouse. J Appl Physiol 2018;124:684-95.

40. Niu Z, Shi Q, Zhang W, Shu Y, Yang N, Chen B, et al. Caspase-1 cleaves PPARgamma for potentiating the pro-tumor action of TAMs. Nat Commun 2017;8:766

41. Chang JS, Ha K. A truncated PPAR gamma 2 localizes to mitochondria and regulates mitochondrial respiration in brown adipocytes. PLoS One 2018; 13:e0195007.

42. Guilherme A, Tesz GJ, Guntur KV, Czech MP. Tumor necrosis factor-alpha induces caspase-mediated cleavage of peroxisome proliferator-activated receptor gamma in adipocytes. J Biol Chem 2009;284:17082-91.

43. Berger J, Moller DE. The mechanisms of action of PPARs. Annu Rev Med 2002;53:409-35.
44. Chiarelli F, Di Marzio D. Peroxisome proliferator-activated receptorgamma agonists and diabetes: current evidence and future perspectives. Vasc Health Risk Manag 2008;4:297-304

45. Moore-Carrasco R, Figueras M, Ametller E, López-Soriano FJ, Argilés JM, Busquets S. Effects of the PPARgamma agonist GW1929 on muscle wasting in tumour-bearing mice. Oncol Rep 2008;19:253-6.

46. Jiang F, Zhang Z, Zhang Y, Pan X, Yu L, Liu S. L-Carnitine ameliorates cancer cachexia in mice partly via the carnitine palmitoyltransferase-associated PPAR- $\gamma$ signaling pathway. Oncol Res Treat 2015;38:511-6.

47. Goncalves MD, Hwang SK, Pauli C, Murphy CJ, Cheng Z, Hopkins BD, et al. Fenofibrate prevents skeletal muscle loss in mice with lung cancer. Proc Natl Acad Sci U S A 2018;115:E743-E52.

48. Wahli W, Braissant O, Desvergne B. Peroxisome proliferator activated receptors: transcriptional regulators of adipogenesis, lipid metabolism and more... Chem Biol 1995;2:261-6.

49. Tontonoz P, Hu E, Spiegelman BM. Stimulation of adipogenesis in fibroblasts by PPAR gamma 2, a lipid-activated transcription factor. Cell 1994;79:1147-56.

50. Fenner MH, Elstner E. Peroxisome proliferator-activated receptor-gamma ligands for the treatment of breast cancer. Expert Opin Investig Drugs 2005 14:557-68

51. Mueller E, Sarraf P, Tontonoz P, Evans RM, Martin KJ, Zhang M, et al Terminal differentiation of human breast cancer through PPAR gamma. Mol Cell 1998;1:465-70.

52. Burnstein HJ, Demetri GD, Mueller E, Sarraf P, Spiegelman BM, Winer EP Use of the peroxisome proliferator-acivated receptor gamma ligand troglitazone as treatment for refractory breast cancer: a phase II study. Breast Cancer Res Treat 2003;79:391-7.

53. Berchtold MW, Brinkmeier H, Müntener M. Calcium ion in skeletal muscle: its crucial role for muscle function, plasticity, and disease. Physiol Rev 2000;80:1215-65.

54. Close RI. Dynamic properties of mammalian skeletal muscles. Physiol Rev 1972;52:129-97.

55. Fontes-Oliveira CC, Busquets S, Toledo M, Penna F, Paz Aylwin M, Sirisi S, et al. Mitochondrial and sarcoplasmic reticulum abnormalities in cancer cachexia: altered energetic efficiency? Biochim Biophys Acta 2013;1830 2770-8. 
CHAPTER 3: Skeletal muscle reprogramming by breast cancer regardless of treatment history or tumor molecular subtype

This article represents an extension of the findings presented in Chapter 2 to include an analysis of transcriptomic and proteomic changes in skeletal muscle from patients with all major molecular subtypes of $\mathrm{BC}$, as well as an analysis of clinical parameters governing muscle's molecular response to BC. We report that patients with all molecular subtypes of BC exhibit similar pathway-level dysregulation in their skeletal muscle, with the common alterations indicating significant disruptions in metabolism of carbohydrates, protein, and lipids. These alterations do not appear to be related to prior treatments, as treatment-naïve individuals exhibit similar changes to patients who underwent neoadjuvant therapies.

In this project, I conducted the majority of bioinformatics and statistical analyses, carried out all Western blots, created the HEK-PPRE reporter cell line, completed most of the in vitro assays, and was the main contributor to the manuscript text.

This article is currently undergoing major revisions with npj Breast Cancer as of April 10, 2020. 
Skeletal muscle reprogramming by breast cancer regardless of treatment history

\section{or tumor molecular subtype}

Hannah E. Wilson (1,2), David A. Stanton (3), Cortney Montgomery (2), Aniello M. Infante (4), Matthew Taylor (5), Hannah Hazard-Jenkins (6), Elena N. Pugacheva (2,7), and Emidio E. Pistilli* $(2,3,8,9)$

1. MD/PhD Medical Scientist Program, West Virginia University School of Medicine, Morgantown, WV, 26506.

2. Cancer Institute, West Virginia University School of Medicine, Morgantown, WV, 26506.

3. Division of Exercise Physiology, Department of Human Performance, West Virginia University School of Medicine, Morgantown, WV, 26506.

4. Genomics Core Facility, West Virginia University, Morgantown, WV, 26506.

5. West Virginia School of Osteopathic Medicine, Lewisburg, WV, 24901.

6. Department of Surgery, West Virginia University School of Medicine, Morgantown, WV, 26506.

7. Department of Biochemistry, West Virginia University School of Medicine, Morgantown, WV, 26506.

8. Department of Microbiology, Immunology, and Cell Biology, West Virginia University School of Medicine, Morgantown, WV, 26506.

9. West Virginia Clinical and Translational Sciences Institute, West Virginia University School of Medicine, Morgantown, WV, 26506.

\section{*Corresponding Author:}

Emidio E. Pistilli, PhD

West Virginia University School of Medicine

Morgantown, WV 26506

304-293-0291 (office) 304-293-7105 (fax)

epistilli2@hsc.wvu.edu 


\section{Author Contributions}

Conception and design of the project (HEW, DAS, EEP); Acquisition, analysis and interpretation of data (HEW, DAS, CM, AMI, MT, HHJ, ENP, EEP); Manuscript preparation (HEW, DAS, EEP).

\section{Acknowledgements}

This research was supported by the following: National Institute of General Medical Sciences of the National Institutes of Health under Award Number P20GM121322 (Lockman), American Cancer Society Institutional Research Grant 09-061-04 (Pistilli), the WVCTSI U54GM104942 (Hodder). Authors would like to acknowledge the following WVU Core Facilities for contributing to this work: Genomics Core Facility; Flow Cytometry and Single Cell Core Facility (S10OD016165); Preclinical Tumor Models Core Facility (CA148671, Pugacheva); Mitochondria Core of the WVU Stroke CoBRE (P20GM109098); Mitochondria, Metabolism and Bioenergetics group (R01 HL-128485; Hollander and the Community Foundation for the Ohio Valley Whipkey Trust). Additional acknowledgements to the Mass Spectrometry and Proteomics Resource Laboratory at Harvard University for proteomics analyses and to Dr. Metheny-Barlow at Wake Forest University for providing EO771 cells.

\section{Competing Interests}

The authors declare that there are no competing interests. 


\section{ABSTRACT}

Increased susceptibility to fatigue is a negative predictor of survival commonly experienced by women with breast cancer. Here, we sought to identify molecular changes induced in human skeletal muscle by $\mathrm{BC}$ regardless of treatment history or tumor molecular subtype using RNA-sequencing (RNA-seq) and proteomic analyses. Mitochondrial dysfunction was apparent across all molecular subtypes, with the greatest degree of transcriptomic changes occurring in women with HER2/neu-overexpressing tumors, though muscle from patients of all subtypes exhibited similar pathway-level dysregulation. Interestingly, we found no relationship between anti-cancer treatments and muscle gene expression, suggesting that fatigue is a product of $\mathrm{BC}$ per se rather than clinical history. In vitro and in vivo experimentation confirmed the ability of BC cells to alter mitochondrial function and ATP content in muscle. These data suggest that interventions supporting muscle in the presence of $\mathrm{BC}$-induced mitochondrial dysfunction may alleviate fatigue and improve the lives of women with BC. 


\section{INTRODUCTION}

Muscle dysfunction in individuals with cancer is commonly thought to be a consequence of muscle atrophy, which is a major component of the paraneoplastic syndrome known as cancer cachexia ${ }^{1,2}$. While studies in men with cancer support the claim that muscle functional capacity is dependent on muscle size, women with cancer report a significant degree of muscle dysfunction despite typically remaining weight-stable ${ }^{3-5}$. Muscle dysfunction in breast cancer $(\mathrm{BC})$ commonly presents as a persistent, severe fatigue that frequently contributes to dose reduction or treatment cessation and is an independent predictor of survival in a variety of cancer types, including $B C^{6-11}$. Thus, it is probable that improving muscle fatigue will improve both quality of life and survival in BC.

At present, there are several purported contributory factors for cancer-related fatigue, including immunological responses to tumor growth; side-effects of cancer therapies; depression and/or emotional distress; anemia; hormonal, nutritional, and metabolic disturbances; and inadequate physical activity ${ }^{8,12}$. Pharmacological treatments for cancer-related fatigue exhibit limited and inconsistent success, in part because determining the mechanisms contributing to fatigue in a given patient can be quite challenging, particularly in patients with early-stage disease and those not receiving anti-cancer treatments ${ }^{13}$. Identifying mechanisms of BC-related fatigue that are intrinsic to skeletal muscle, generalizable across BC subtypes, and independent of treatment status could significantly aid in the development of appropriate therapies, which would be applicable to a large number of patients experiencing BC-associated fatigue. 
Our laboratory has recently reported that skeletal muscle of women with $\mathrm{BC}$ exhibits a distinct gene expression signature that is not dependent on molecular subtype ${ }^{14}$. Furthermore, we have identified signaling via the metabolic regulators of the peroxisome-proliferator activated receptor (PPAR) family as potential key mediators of fatigue in women with $B C$ and female mice bearing $B C$ patient-derived orthotopic xenografts (PDOXs) ${ }^{15}$. Our previous analyses did not include women with primary tumors that overexpressed HER2/neu in the absence of estrogen receptor (ER) and progesterone receptor (PR) expression. In the current study, we have expanded our analyses into all molecular subtypes, including both transcriptomic and proteomic analyses of muscle biopsies from patients with HER2/neu-overexpressing tumors, and significantly increased our sample size to create, to our knowledge, the largest study of transcriptomic and proteomic changes in muscle of women with BC. We tested the hypothesis that $\mathrm{BC}$ induces a common molecular response in skeletal muscle that is independent of the molecular subtype of the tumor and the patient's treatment history. 


\section{RESULTS}

Patient characteristics. A total of $51 \mathrm{BC}$ patients representing 4 breast tumor subtypes and 20 non-cancer controls provided pectoralis major muscle biopsies and/or detailed clinical information for use in the present study. There were no differences in mean body mass index (BMI) between non-cancer controls and BC patients; average BMI in the control group was categorized as Class I Obesity and in the BC patients was categorized as Overweight. Additionally, there were no significant differences in the percent $(\%)$ change in $\mathrm{BMI}$, body fat $\%$, or lean body mass between controls and $\mathrm{BC}$ patients. There were no differences in BMI between any of the 4 breast tumor subtypes (Table 1).

Skeletal muscle gene expression profiles. Skeletal muscle biopsies from patients diagnosed with $B C(n=33)$ and non-cancer controls $(n=10)$ were used for RNA-seq, and BC patients were classified based on molecular subtype of their primary tumor, as follows: luminal (ERPR)— positive for estrogen receptor (ER) and progesterone receptor (PR) without overexpression of HER2/neu; HER2 —overexpression of HER2/neu in the absence of ER and PR expression; triple negative (TN)-absence of ER, PR, and HER2/neu expression; and triple positive (TP) — presence of ER and PR expression, and overexpression of HER2/neu (ERPR $n=10$, HER2 $n=5, T N n=9$, TP n=9). Unnormalized gene expression counts are provided for each sample (Supplemental Table 1). Unsupervised clustering and principal component analyses suggested the possibility of clustering based on molecular subtype, particularly with regard to patients with tumors overexpressing HER2/neu in the absence of ER or PR (Figure 1A). Multidimensional scaling (MDS) analysis restricted to 3 dimensions 
revealed that the gene expression profiles of skeletal muscle from patients with ERPR, TP and TN tumors were similar, while the profile from skeletal muscles from patients with HER2/neu-overexpressing tumors was significantly different (Figure 1B). Overall fit of the MDS model was dramatically improved by the use of BC subtype as a covariate (Figure 1B, adjusted $\mathrm{R}^{2}=0.44, \mathrm{p}=0.0001$ ) rather than a model including only binary disease status (Figure 1C, adjusted $R^{2}=0.20, p=0.008$ ), which was already a significant improvement over the null model (Figure 1D).

To address the question of whether this obvious difference in overall muscular gene expression between groups was inherent to differences in the primary tumor type or the myriad of clinical characteristics that could potentially differ between groups, we assessed the relationship between clinical characteristics and skeletal muscle gene expression in the context of a multivariate linear regression model, using the 3 dimensions of the MDS dissimilarity matrix as response variables. Among the various treatment types, body composition, serum albumin, and changes in body mass, the only assessed variable to yield statistical significance at $\alpha=0.05$ when used as a single independent variable was patient group (i.e. Control, ERPR, HER2, TN, TP), with serum albumin nearing statistical significance (Table 2). Notably, chemotherapy, radiotherapy, and hormonal treatments did not correlate with overall gene expression patterns, nor did the patient's trend of weight change over time. Using forward selection, a final model including patient group and serum albumin was identified as the best-fitting model for predicting muscular gene expression from clinical data (Table 3). 
Because serum albumin appeared to provide predictive value for skeletal muscle gene expression data, and because serum albumin is commonly used by oncologists to monitor patients' nutritional status, the relationship between serum albumin and changes in BMI over time were assessed in the group of patients that provided biopsies. There was no correlation observed between serum albumin at the date of biopsy collection and the individual's rate of weight change over time (Figure2A). Similar results were obtained in a retrospective chart review of 3,001 patients with $\mathrm{BC}$. While there was a statistically significant correlation between a patient's first record of serum albumin and the patient's rate of weight change in this large sample (Figure 2B), the effect size may well be clinically insignificant $(R=0.094)$. The average daily weight change was negligible in women with normal serum albumin as well as those with low serum albumin $\left(<3.4 \mathrm{~g} \cdot \mathrm{dL}^{-1}\right)$, with both groups having means within one standard deviation of 0 (Figure $\mathbf{2 C}$ ). For a patient to be considered cachectic by traditional standards, an average daily weight loss of at least $0.027 \%$ would be required to lose $5 \%$ of their weight in 6 months ${ }^{1,2}$. In our large cohort, a logistic regression analysis was conducted using a threshold of $3.4 \mathrm{~g} \cdot \mathrm{dL}^{-1}$ serum albumin to predict whether a patient would exhibit this rate of weight change. In this analysis, omnibus model fit was significant at $a<0.05$, though effect size as determined by

Nagelkerke's pseudo- $R^{2}$ was very small and indicates a very weak predictive value $\left(R^{2}\right.$ $=0.08$ ). Additionally, a threshold of $3.4 \mathrm{~g} \cdot \mathrm{dL}^{-1}$ serum albumin was only $40 \%$ sensitive to identifying this level of weight change and only yielded a positive predictive value of $17.3 \%$. In other words, $60 \%$ of $\mathrm{BC}$ patients exhibiting a rate of weight loss consistent with cachexia had normal serum albumin, while BC patients with serum albumin $<3.4 \mathrm{~g}$ 
$\cdot \mathrm{dL}^{-1}$ only had a $17.3 \%$ chance of exhibiting a rate of weight change consistent with cachexia.

Differential gene expression analysis by subtype. Differentially expressed genes (DEGs) within skeletal muscle were first identified by comparing BC patients by subtype to control. Considerable overlap of DEGs was observed between the 4 breast tumor subtypes. Of the 3,468 genes identified as differentially expressed in at least one subtype, only $7(0.2 \%)$ were unique to ERPR patients, $173(5.0 \%)$ to TN patients, and $80(2.3 \%)$ to TP patients. However, 2,410 genes $(70 \%)$ were unique to HER2 patients, and only $8(0.23 \%)$ were differentially expressed in all patient groups (Figures $3 \mathbf{A}$ and 3B). These observations were quantified and reveal that there was an approximately 2fold fewer-than-expected number of unique DEGs in muscle of patients with ERPR, TP and TN tumors if the DEGs were independent of subtype. Specifically, one would expect 14,186 , and 508 unique DEGs in each subtype, respectively, whereas we actually observed 7,80 , and 173 DEGs in these groups. Further, HER2 patients' muscle exhibited 2-fold fewer-than-expected DEGs shared with any combination of two other subtypes (Observed $=5+2+84=91$; Expected total $=203$; overall $X^{2}$ on 6 degrees of freedom $\left.=1224, p=3 \times 10^{-261}\right)$. This indicates that the ERPR, TP, and TN groups share a greater number of DEGs in skeletal muscle than one would expect if the DEGs were independent of subtype and in contrast, muscle from HER2 patients does not exhibit the same similarity to the other subtypes in terms of shared DEGs (Figure 3C). Collectively, these data demonstrate that transcriptional responses in skeletal muscle of patients with ERPR, TP and TN tumors are highly similar, in support of previous data from our laboratory ${ }^{14,15}$. Furthermore, the transcriptional responses in muscles from patients with 
HER2/neu-overexpressing tumors partially overlap with the other subtypes, but exhibit a significant contrast to the other 3 subtypes, suggesting that this tumor type is associated with a unique transcriptional adaptation within skeletal muscle.

Pathway analysis by subtype. The Broad Institute's Gene Set Enrichment Analysis (GSEA) tool ${ }^{16}$ was used to infer pathway-level dysregulation based on trends in transcriptomic changes, assessing all pathways in the Kyoto Encyclopedia of Genes and Genomes (KEGG) ${ }^{17}$. Identified pathways were shared between all breast tumor subtypes to a greater extent than individual genes. Of the 70 pathways identified as dysregulated in any subtype, $19(27.1 \%)$ pathways were identified as significantly dysregulated in all 4 breast tumor subtypes relative to control. $2(2.9 \%)$ pathways were uniquely dysregulated in muscle from patients with TP tumors, $10(14.2 \%)$ in patients with ERPR tumors, 7 (10.0\%) unique to TN patients, and 4 (5.7\%) uniquely dysregulated in patients with HER2/neu-overexpressing tumors (Figures 4A, 4B, 4C). Chi-square analysis to test whether the number of pathways shared between subtypes differed between groups was non-significant (overall $\chi^{2}$ on 6 degrees of freedom $=3.67$, $p=0.72$ ), indicating that the four $B C$ subtypes share a common core of dysregulated pathways (Figure 4C). The 19 overlapping pathways clearly show a substantial degree of metabolic alteration, including pathways involved in carbohydrate, lipid, and protein metabolism (Figure 4D). While the enrichment of Alzheimer's, Parkinson's, and Huntington's diseases may seem unusual, these pathways include a large number of mitochondrial genes, explaining why these pathways cluster with Oxidative Phosphorylation. The enrichment of the KEGG Peroxisome pathway is significant, as our laboratory has previously identified the PPAR proteins as likely upstream regulators 
of muscle fatigue in $\mathrm{BC}{ }^{14,15}$. Though our multivariate linear regression model did not show neoadjuvant therapies to predict overall gene expression, the upregulation of transcripts in the KEGG p53 signaling pathway is likely a response to chemotherapy and/or radiotherapy.

HER2 patient proteomics. Because the HER2 patient group exhibited the greatest degree of transcriptomic dysregulation, muscle biopsies from these patients $(n=5)$ were selected for proteomic analysis and compared to control surgical patients $(n=5)$. Unnormalized protein abundance estimates are provided for each sample (Supplemental Table 2). Scaled, log-transformed expression data from proteomic analyses were correlated with scaled, log-transformed RNA-seq expression data in a gene-wise fashion. 8/8 individuals with matched RNA-seq and proteomic analyses were found to have moderately strong correlation (Pearson's $\mathrm{R}$ range $0.48-0.52$, Supplemental Figure 1). A total of 1,555 unique proteins were detected across all samples, 1,259 of those at a high confidence level, with most proteins being detected in all 10 samples (Figure 5A). Differential expression analysis detected only a small number of significantly differentially expressed proteins (FDR $<0.05$, Figure 5B), though there are obvious physiological implications in the small set. The six downregulated differentially expressed proteins (DEPs) were all identified as mitochondrial components (Figure $\mathbf{5 C}$ ), representing a statistically significant enrichment of mitochondrial components in this small set of DEPs (Figure 5D, $p=3.2 x$ $\left.10^{-6}\right)$. Expanding our analysis to both significant DEPs and insignificant trends in protein expression, a strong signal for aberrant mitochondrial function was once again 
detected. Nearly every protein involved in the mitochondrial electron transport chain was quantified as having a lower level of protein expression in the HER2 patients relative to controls (Figure 5E, Supplemental Table 3), including proteins encoded by both nuclear DNA and mitochondrial DNA. GSEA results from the proteomic data were highly similar to those obtained in the RNA-seq analysis (Supplemental Figure 2), with significantly affected pathways including Oxidative Phosphorylation (NES $=-2.97$, FDR $<0.001)$, Citrate Cycle (NES $=-2.12$, FDR $<0.001)$, Peroxisome $($ NES $=-1.71$, FDR $=$ 0.02), and Fatty Acid Metabolism (NES $=-1.66, F D R=0.27$ ), among other pathways. Two mitochondrial proteins identified as significantly differentially expressed in the HER2 patient group were selected for validation by Western blotting in a completely independent cohort of patients, without regard to patient molecular subtype. Both proteins assessed were confirmed to have lower abundance in the BC group compared to control (Figure 5F).

Subtype-independent DEG analysis and experimental validation. To cross-validate our RNA-seq GSEA results using an independent software, pathway-level enrichment analysis was conducted using the list of genes that were significantly differentially expressed when comparing all BC patients to control (FDR $<0.10)$. In this analysis, we observed a strong signal indicating aberrant mitochondrial function across multiple databases queried. For example, three of the top four enriched pathways identified when querying the WikiPathways 2019 Human database included Electron Transport Chain System in Mitochondria (WP111), Oxidative Phosphorylation (WP623), and Mitochondrial Complex I Assembly (WP477, Figure 6A); and the three most enriched 
cellular components from the 2018 Gene Ontology project were all mitochondrial components (Figure 6B).

To test this prediction of altered mitochondrial function in tissues distant from the primary tumor in vivo, we generated 6 female BC-PDOX mice and isolated live mitochondria from their skeletal muscle at euthanasia for quantification of ATP content. We found a significant reduction in ATP content within both of the two major skeletal muscle mitochondrial subpopulations, the interfibrillar mitochondria (IFM) and subsarcolemmal mitochondria (SSM), relative to female control animals (Figure 6C). We additionally quantified ATP content in snap-frozen muscle biopsies from a large group of women with BC and control patients. Consistent with our in silico predictions and murine studies, bulk muscle from BC patients was quantified as having significantly less ATP per gram of protein than control muscle, though the large degree of variability in absolute quantifications should be noted (Figure 6D). In vitro assays were conducted to determine whether mitochondrial dysfunction in muscle is a direct response to $\mathrm{BC}$ secreted factors or an indirect response mediated by other tissues. In support of a direct response, conditioned media from the luminal EO771 BC cell line and the HER2/neu overexpressing NF639 line significantly repressed aerobic ATP production in differentiated $\mathrm{C} 2 \mathrm{C} 12$ myotubes, whereas media conditioned by either the normal mammary epithelial cell line EpH4-EV or $\mathrm{C} 2 \mathrm{C} 12$ myoblasts did not (Figure 6E). To validate the role of the PPAR signaling proteins in mediating the systemic response to BC-secreted factors, conditioned media was isolated from EO771 and NF639 cells and applied to HEK293 cells stably expressing a PPAR-responsive promoter driving GFP 
expression. Media conditioned by both BC cell lines significantly repressed GFP intensity relative to reporter cell conditioned media, while media conditioned by a normal mammary epithelial cell line did not alter GFP signal relative to control (Figure 6F). These data indicate that $\mathrm{BC}$ cells secrete a substance that is capable of directly influencing metabolic function in skeletal muscle, which may be related to a repression of PPAR-mediated transcriptional activity. 


\section{DISCUSSION}

$\mathrm{BC}$-induced muscle dysfunction is a common problem of unclear etiology with few therapeutic options. Here we sought to identify possible mechanisms of fatigue that are generalizable across BC subtypes and are independent of treatment status by assessing statistical relationships between patients' clinical characteristics and overall skeletal muscle molecular composition.

In support of our laboratory's previous publications ${ }^{14,15}$, we found that women with three molecular subtypes of BC, those being ERPR, TN, and TP BC, exhibit overall similarity in muscular gene expression. Remarkably, patients with tumors overexpressing HER2/neu in the absence of ER and PR expression exhibited a markedly different muscular gene expression profile. However, gene expression data from all subtypes pointed to similar pathway-level dysregulation, indicating that the mechanisms leading to muscle fatigue in $\mathrm{BC}$ patients may indeed be generalizable across subtypes, although we acknowledge this conclusion is based on a limited sample size across individual BC subtypes. In assessing the commonalities across patients, we observed significant dysregulation of metabolic pathways in all groups of BC patients, and proteomic analysis of patients with HER2/neu-overexpressing tumors showed decreased abundance of nearly all proteins involved in the mitochondrial electron transport chain. Because mitochondrial density in skeletal muscle has been shown to correlate with abundance of ETC complex proteins and skeletal muscle oxidative capacity ${ }^{18}$, it is likely that $\mathrm{BC}$ patients also have decreased mitochondrial densityl in their skeletal muscle as well as decreased oxidative capacity. We propose then that BC-secreted factors induce muscle dysfunction by abrogating oxidative 
capacity via alteration of mitochondrial biogenesis, mitophagy, or fission/fusion dynamics. Both in vitro and in vivo assays confirm that factors from BC cells alter skeletal muscle ATP content and/or aerobic ATP production, perhaps via dysregulation of the PPAR-signaling pathway.

Our laboratory's interest in the PPAR family of proteins arose from our previous RNAseq analysis of muscle from BC patients and PDOX-bearing mice, which our group found to recapitulate the clinical phenotype of increased muscle fatigue without muscle atrophy or bodyweight loss ${ }^{15}$. These proteins have demonstrated roles in whole-body energy regulation, are critical regulators of mitochondrial function in multiple tissues, and are targets of multiple

FDA-approved agents in the treatment of type 2 diabetes and hyperlipidemia. Among the three PPAR isoforms, we identified PPARG as a key regulator in BC-induced muscle dysfunction observed in PDOX mice ${ }^{15}$. PPARG is a ligand-activated nuclear receptor that, upon activation by a variety of endogenous and synthetic lipids, forms complexes with retinoid $\mathrm{X}$ receptor $(\mathrm{RXR})$ and cofactors such as the peroxisome proliferator-activated receptor- $\gamma$ coactivator $1 \alpha(P G C 1 \alpha)$, and stimulates transcription of downstream genes. This relationship with PGC1 $\alpha$ is particularly relevant, as PGC1 $\alpha$ is known to be a master regulator of mitochondrial biogenesis in several tissues, including skeletal muscle ${ }^{19}$. PGC1 $\alpha$ also participates in the regulation of other metabolic processes, including gluconeogenesis, muscle fiber-type specification, and control of antioxidant expression ${ }^{20-22}$. Therefore, the interaction between PPARG and PGC1a clearly has the potential to impact muscle function through mitochondrial mechanisms. In addition to the potential development of muscle-intrinsic mitochondrial dysfunction, a 
systemic consequence of dysregulated PPARG is the development of insulin resistance $(\mathrm{IR})^{23}$, which is commonly associated with type 2 diabetes, obesity, and metabolic syndrome, and appears to have a bidirectional relationship with $B C$, with individuals with $\mathrm{IR}$ at greater risk of $\mathrm{BC}$ and $\mathrm{BC}$ survivors at an increased risk of $\mathrm{IR}^{24,25}$. We propose that the development of mitochondrial dysfunction and IR, secondary to muscular PPARG downregulation by $\mathrm{BC}$, creates an environment that facilitates the development of muscle fatigue through several mechanisms, including decreased mitochondrial ATP production as well as dysregulated glucose and lipid metabolism. Pharmacological restoration of PPARG function results in the induction of a number of genes involved in insulin signaling, as well as glucose and lipid metabolism, and PPAR agonist drugs including the thiazolidinediones have shown a remarkable ability to restore insulin sensitivity in insulin-resistant conditions. Because our predictions have implicated many related metabolic pathways, we hypothesize that the development of $B C$ induced muscle fatigue constitutes a pathology similar to type 2 diabetes/metabolic syndrome. If repression of PPAR signaling is indeed central to BC-induced muscle dysfunction, the numerous FDA-approved PPAR-agonists could address this unmet need in clinical oncology.

A particularly surprising result in the present study was that no clinical data aside from BC molecular subtype exhibited significant correlation with skeletal muscle gene expression, including TNM staging and history of chemotherapy, radiation, or immunotherapy. Additionally, patients in the HER2 group consistently exhibited a decreased abundance of mitochondrial proteins in their muscle tissue, and importantly, we observed these responses consistently across all five biopsies from this patient 
group despite significant differences in anti-cancer treatment history. At the date of biopsy collection, one patient was entirely treatment naïve, one patient had completed chemotherapy for BC 10 years prior, and the remaining 3 patients received different multi-agent neoadjuvant chemotherapy in the months prior to surgery. Therefore, we propose that changes in skeletal muscle physiology seen in BC are due to tumorderived factors rather than side effects of therapies or other patient-specific factors. This hypothesis is supported by our in vitro conditioned media experiments where tumorderived factors directly repressed mitochondrial respiratory capacity in differentiated muscle cells. Additionally, neither weight loss nor body composition were predictive of skeletal muscle gene expression, suggesting that gene expression changes observed in $\mathrm{BC}$ patients are unrelated to muscle atrophy or cachexia and may instead be reflective of muscle dysfunction. Therefore, the molecular alterations we highlight suggest that skeletal muscle is responding at the early stages of breast tumor growth, at a time when patients would be considered "pre-cachectic", based on Fearon's cachexia continuum ${ }^{1}$. Clinicians should be aware that patients with BC may not have dramatic changes in body weight and/or muscle mass during tumor growth or tumor-directed therapies. However, this does not necessarily imply that muscle dysfunction, in the form of persistent fatigue, is not adversely affecting patients' quality of life.

Because albumin is often used by physicians as a measure of patients' nutritional status and mortality risk ${ }^{26-29}$, we assessed serum albumin in our patient cohort and assessed its relationship to skeletal muscle gene expression and weight change. In our analysis, serum albumin was not found to be predictive of weight change, and in a larger sample was found to be only weakly predictive of cachexia risk. Yet, it was 
unexpectedly predictive of skeletal muscle gene expression. This indicates that serum albumin may be a useful biomarker of muscle function during pre-cachexia, a possibility supported by previous literature connecting serum albumin with muscle strength ${ }^{30}$ and insulin resistance ${ }^{31}$ in other clinical contexts. Prospective studies directly addressing the relationship between skeletal muscle function, gene expression, and serum albumin would be required to validate the clinical utility of serum albumin in identifying those at risk of cancer-induced muscle dysfunction.

In line with our previous publications ${ }^{14,15}$, we report that transcriptional responses to the ERPR, TP and TN subtypes of BC are similar in terms of skeletal muscle gene expression, while muscle biopsies from patients with tumors overexpressing HER2/neu in the absence of ER and PR exhibit an unusual degree of uniqueness in terms of gene expression. We identified a strong signal for BC-induced mitochondrial dysfunction in $\mathrm{BC}$ patients, $\mathrm{PDOX}$-bearing animals, and in in vitro assays, and proteomic analysis showed decreased protein abundance of nearly all components of the mitochondrial electron transport chain in muscle biopsies from patients with HER2/neu-overexpressing tumors relative to control. Further, we found no relationship between various BC-related treatments (surgery, chemotherapy, radiotherapy) and changes in skeletal muscle gene expression. However, serum albumin was predictive of skeletal muscle gene expression without being predictive of weight loss, suggesting that serum albumin may be a useful indicator of BC-induced skeletal muscle dysfunction. Overall, these data indicate that all $B C$ is associated with dysfunction in mitochondrial respiration in skeletal muscle independent of molecular subtype, and this effect appears to be independent of anticancer treatments. These findings call for prospective studies assessing interventions to 
support skeletal muscle function in the presence of BC-induced mitochondrial dysfunction. 


\section{METHODS}

Patient information. Conduct of research involving human patients at West Virginia University is guided by the principles set forth in the Ethical Principles and Guidelines for the Protection of Human Subjects of Research (Belmont Report) and is performed in accordance with the Department of Health and Human Services policy and regulations. A total of 71 female surgical patients provided informed consent for inclusion in this study (control $n=20 ; B C n=51$ ). Informed written consent was obtained from each subject or each subject's guardian. Individuals could be included in this study if they 1) were deemed to have operable disease or were undergoing a diagnostic biopsy, 2) had been diagnosed with, or were suspected to have, invasive adenocarcinoma of the breast, 3) were over 21 years old, and 4) provided informed consent. Women with BC provided muscle biopsies from the pectoralis major muscle intraoperatively at the time of mastectomy, and control patients provided pectoralis major muscle samples intraoperatively during other breast surgeries. Women with BC were classified into four molecular subtypes based on immunohistochemical staining of their primary tumors: $\operatorname{ERPR}(n=20), \operatorname{HER} 2(n=9), \operatorname{TN}(n=11)$, or TP $(n=11)$.

Information on BMI at multiple time points was collected in 12 control and $50 \mathrm{BC}$ patients. The mean number of days a consented patient was followed was $170+/-214$ (control median=170, ERPR median=36, HER2 median=146, TP median=140, TN median=180). Detailed body composition analyses were acquired in 8 control and 35 BC patients using a bioelectrical impedance scale (Tanita: model SC-240). Serum albumin levels were acquired in 3 control and 49 BC patients (ERPR $n=18$; HER2 $n=9$; 
TP $n=11 ; T N n=10$ ). For RNA-Seq analyses, biopsies were used from 10 control and 33 BC patients (ERPR $n=10$; HER2 $n=5 ;$ TP $n=9 ;$ TN $n=9$ ).

In a separate analysis of de-identified electronic medical records (EMRs) from female BC patients, body mass and standing height data were acquired for 5,201 individuals for calculation of BMI. Within this population, final analyses were completed in 3,001 patients having at least two measurements for weight in addition to at least one record for serum albumin.

RNA-Sequencing. Pectoralis major muscle biopsies were acquired intraoperatively and stored in RNA stabilization reagent (Invitrogen, Thermo Fisher, San Jose, CA) overnight at $4^{\circ} \mathrm{C}$ and then at $80^{\circ} \mathrm{C}$ until processing. RNA was isolated, assessed for quality, and utilized to construct libraries for RNA-Seq as previously reported ${ }^{15}$. Completed libraries were sequenced on one lane of the HiSeq 1500 with PE50 bp reads, with approximately 15 million reads per sample. Subsequently, Salmon was used for transcript-level abundance estimation, with both gcBias and seqBias set, and libType $\mathrm{A}^{32}$, using the GRCh38 genome assembly. Between $5-10 \%$ of reads in each sample were unable to be mapped and were excluded from analyses reported here. Transcript-level abundance estimates were summarized to the gene-level using tximport ${ }^{33}$. The resulting counts matrix was scaled to library size using edge $R^{34}$ and filtered to remove genes without a counts-per-million (CPM) value $>1$ in 3 or more samples. Logtransformed CPM values for the 10,000 genes with highest variance were used as input for hierarchical clutering using gplots ${ }^{35}$. CPM values of all genes with CPM $>1$ in at least 3 samples were scaled and log-transformed prior to distance matrix computation, which was then input for classical multidimensional scaling with $\mathrm{k}=3$ and visualized in 3- 
dimensions using $\mathrm{car}^{36}$. This 3-dimensional distance matrix (3D-MDS) was then utilized as 3 response variables representing gene expression in the multivariate regression analysis to assess statistical relationships between clinical parameters and overall gene expression. Differential gene expression analysis was conducted using DESeq2 ${ }^{37}$. Both the multivariate regression analysis and differential expression analysis are further described below, within "RNA-Seq and clinical correlates" in the Statistical Analyses section. Power was determined post hoc to be $88 \%$ using RnaSeqSampleSize ${ }^{568}$ with 1000 repetitions, $f=0.1$, and $r h o=2$.

Proteomics. Sample Preparation: Pectoralis major muscle biopsies from $\mathrm{n}=5$ female patients with HER2/neu-overexpressing $B C$ and $n=5$ control breast surgical patients were acquired intraoperatively and stored in RNA-later at $80^{\circ} \mathrm{C}$ until processing ${ }^{39}$. Muscle biopsy samples weighing approximately $50 \mathrm{mg}$ were sent on dry ice to the Mass Spectrometry and Proteomics Resource Laboratory at Harvard University and processed according to established protocols ${ }^{40,41}$. Briefly, biopsies were lysed in Covaris ${ }^{\circledR}$ microTUBE- 15 (Woburn, MA) microtubes with Covaris ${ }^{\circledR}$ TPP buffer, using the Covaris S220 Focusedultrasonicator instrument with $125 \mathrm{~W}$ power over 180 s with $10 \%$ max peak power. Samples were then chloroform/methanol precipitated, filtered, reduced, alkylated, and finally digested overnight at $38^{\circ} \mathrm{C}$ in a solution containing triethylammonium bicarbonate and Promega ${ }^{\circledR}$ Sequencing Grade Trypsin. Digested peptides were then incubated with tandem mass tag, using different tags for each sample. 
LC-MS/MS: The 10 samples were pooled in equal amounts and fractionated into 10 fractions. LC-MS/MS was performed on an Orbitrap Lumos (Thermo Fisher) equipped with EASYLC1000 (Thermo Fisher). Peptides were separated onto a $100 \mu \mathrm{m}$ inner diameter microcapillary column packed first with C18 Reprosil resin (5 $\mu \mathrm{m}, 100 \AA$, Dr. Maisch $\mathrm{GmbH}$, Germany) followed by analytical column of Reprosil resin $(1.8 \mu \mathrm{m}, 200 \AA$, Dr. Maisch $\mathrm{GmbH}$, Germany). Separation was achieved through applying a gradient from 5- 27\% acetonitrile in $0.1 \%$ formic acid over $90 \mathrm{~min}$ at $200 \mathrm{nl} \cdot \mathrm{min}^{-1}$. Electrospray ionization was enabled through applying a voltage of $1.8 \mathrm{kV}$ using a homemade electrode junction at the end of the microcapillary column and sprayed from fused silica pico tips (New Objective, MA). The LTQ Orbitrap Lumos was operated in data-dependent mode for the mass spectrometry methods. The mass spectrometry survey scan was performed in the Orbitrap in the range of $395-1,800 \mathrm{~m} / \mathrm{z}$ at a resolution of $6 \times 10^{4}$, followed by the selection of the twenty most intense ions (TOP20) for collision induced dissociation (CID) in the Ion trap using a precursor isolation width window of $2 \mathrm{~m} / \mathrm{z}$, AGC setting of 10,000 , and a maximum ion accumulation of $200 \mathrm{~ms}$. Singly charged ion species were not subjected to CID fragmentation. Normalized collision energy was set to $35 \mathrm{~V}$ and an activation time of $10 \mathrm{~ms}$. Ions in a $10 \mathrm{ppm} \mathrm{m} / \mathrm{z}$ window around ions selected for MS2 were excluded from further selection for fragmentation for $60 \mathrm{~s}$. The same TOP20 ions were subjected to higher-energy collisional dissociation (HCD) MS2 event in Orbitrap part of the instrument. The fragment ion isolation width was set to $0.7 \mathrm{~m} / \mathrm{z}$, AGC was set to 50,000 , the 
maximum ion time was $200 \mathrm{~ms}$, normalized collision energy was set to $27 \mathrm{~V}$ and an activation time of $1 \mathrm{~ms}$ for each HCD MS2 scan.

Mass spectrometry analysis: Raw data were submitted for analysis in Proteome Discoverer 2.2 (Thermo Scientific). Assignment of MS/MS spectra was performed using the Sequest HT algorithm by searching the data against a protein sequence database including all entries from Uniprot_Human2016_SPonly database as well as other known contaminants such as human keratins and common lab contaminants. Sequest HT searches were performed using a 20 ppm precursor ion tolerance and requiring each peptides $\mathrm{N}-/ \mathrm{C}$ termini to adhere with trypsin protease specificity, while allowing up to two missed cleavages. 6-plex TMT tags on peptide $\mathrm{N}$ termini and lysine residues $(+229.162932 \mathrm{Da})$ were set as static modifications while methionine oxidation (+15.99492 Da) was set as a variable modification. A MS2 spectra assignment false discovery rate (FDR) of $1 \%$ on both protein and peptide level was achieved by applying the target-decoy database search. Filtering was performed using a Percolator ${ }^{42}$ (64bit version). For quantification, a $0.02 \mathrm{~m} / \mathrm{z}$ window centered on the theoretical $\mathrm{m} / \mathrm{z}$ value of each the six reporter ions and the intensity of the signal closest to the theoretical $\mathrm{m} / \mathrm{z}$ value was recorded. Reporter ion intensities were exported using Proteome Discoverer 2.2.

Western blotting analysis. Pectoralis major muscle biopsies from $\mathrm{n}=13$ female patients with $B C$ and $n=10$ control breast surgical patients were snap-frozen and stored at $-80^{\circ} \mathrm{C}$ until processing. Biopsies from $n=7$ female patients with $B C$ and $n=7$ control breast surgical patients were selected from this pool using stratified random sampling by patient group (i.e. BC or $\mathrm{CON}$ ) without regard to patient molecular subtype. Protein 
homogenates were made using a $5 \mathrm{~mL}$ Wheaton tissue grinder (DWK Life Sciences Inc., Millville, NJ, USA) in a solution of $20 \mathrm{mM}$ Tris $\mathrm{HCl}(\mathrm{pH}=7.4), 150 \mathrm{mM} \mathrm{NaF}, 1 \mathrm{mM}$ EDTA, $1 \%$ Triton X-100, and 10\% glycerol. Homogenates were cleared via brief centrifugation, and protein concentration was quantified using DC ${ }^{\mathrm{TM}}$ Protein Assay (BioRad, California, USA) according to manufacturer's protocol. $12 \mu \mathrm{g}$ of total protein was loaded per well and resolved in NuPAGE Novex 4-12\% Bis-Tris Gels (ThermoFisher Scientific). Proteins were transferred to nitrocellulose membrane, Ponceau stained to assess loading, destained, blocked in $1 \mathrm{X}$ tris-buffered saline (TBS), $0.1 \%$ Tween20, $5 \%$ milk, then incubated with primary antibody overnight at $4^{\circ} \mathrm{C}$. Membranes were then washed prior to application of appropriate secondary antibody (ThermoFisher Scientific), and again prior to application of Pierce ECL Western Blotting Substrate (ThermoFisher Scientific). Relative band intensity was quantified using the GE Amersham Imager 600 (GE Healthcare Life Sciences, Marlborough, MA, USA). GAPDH and Ponceau staining are presented as loading controls but were not used to normalize densitometry data. Primary antibodies included CKMT2 (ThermoFisher Scientific, \#PA528591), COX7A1 (ThermoFisher Scientific, \#PA5-67696), and GAPDH (Cell Signaling Technology, Massachusetts, USA, \#2118S).

BC patient muscle ATP quantification. Pectoralis major muscle biopsies from $n=13$ female patients with $B C$ and $n=10$ control breast surgical patients were snap-frozen and stored at $-80^{\circ} \mathrm{C}$ until processing as described under "Western blotting analysis." All available snap-frozen muscle biopsies were included in analysis of ATP content. ATP content was quantified using the ENLITEN® ATP Assay System Bioluminescence 
Detection Kit (Promega, Wisconsin, USA) according to manufacturer's protocol, using the manufacturer's recommended standard curve for calculation of absolute ATP content from luminescence intensity and The FlexStation ${ }^{\circledR} 3$ Multi-Mode Microplate Reader (Molecular Devices $₫$, California, USA). ATP content was normalized to protein concentration, quantified using the DC ${ }^{\mathrm{TM}}$ Protein Assay (Bio-Rad, California, USA).

BC-PDOX skeletal muscle mitochondria metabolic analysis. Animal experiments were approved by the WVU Institutional Animal Care and Use Committee. BC-PDOX mice were created by implanting human $\mathrm{BC}$ tumor fragments into the mammary fat pad of female NOD.CG-Prkdscid II2rgtm1 Wjl/SzJ/ 0557 (NSG) mice (n=6), as described previously ${ }^{15}$. PDOX-bearing animals were euthanized approximately 30 days after reaching a tumor volume of $200 \mathrm{~mm}^{3}$. Control female NSG mice of similar age $(n=4)$ were euthanized at the same time as tumor-bearing animals and tissues were processed identically in both groups. Immediately after death, both quadriceps muscles from each mouse were quickly removed and interfibrillar (IFM) and subsarcolemmal (SSM) mitochondria were isolated separately according to previously described methods ${ }^{43-46}$, combining the two muscles to obtain sufficient tissue for downstream applications. Mitochondrial isolates were stored at $-80^{\circ} \mathrm{C}$ until analysis. ATP content was quantified in each mitochondrial subpopulation using the ENLITEN® ATP Assay System Bioluminescence Detection Kit (Promega) according to manufacturer's protocol with minor modifications, as follows. Mitochondrial isolates were lysed in $1 \%$ trichloroacetic acid for 5 minutes then diluted $1: 10$ in $0.1 \mathrm{~mol} \cdot \mathrm{L}^{-1}$ tris base $(\mathrm{pH}=7.8)$. The resulting samples were loaded into a black-walled microplate, in duplicate, and mixed 1:1 with 
luciferase/luciferin reagent. Luminescence intensity was immediately read using the FlexStation ${ }^{8} 3$ Multi-Mode Microplate Reader (Molecular Devices ${ }^{\circledR}$, California, USA). Luminescence intensity values were blank-corrected and normalized to account for differing protein concentrations, which were quantified using the DC ${ }^{\mathrm{TM}}$ Protein Assay (Bio-Rad, California, USA).

Cell culture. All cell lines were obtained from ATCC (Virginia, USA) with the exception of EO771s. Cells were cultured in Gibco DMEM (Thermo Fisher, Massachusetts, USA) supplemented with $10 \%$ heat inactivated fetal bovine serum (Atlanta Biologicals, Georgia, USA) and Gibco penicillin/streptomycin (Thermo Fisher) at $37^{\circ} \mathrm{C}$ with $6 \% \mathrm{CO}_{2}$. Cell lines utilized include EpH4-EV (immortalized normal murine mammary epithelium), EO771 (murine luminal BC), NF639 (murine HER2/neu-overexpressing BC), HEK293 (human embryonic kidney), and C2C12 (murine myoblasts).

In vitro conditioned media (CM) metabolic analysis. $\mathrm{C} 2 \mathrm{C} 12$ cells were plated into Agilent Seahorse XF24 (Agilent Technologies, California, USA) plates and differentiated by confluence for 3 days. Meanwhile, EpH4-EV, EO771, NF639, and C2C12 cells were plated at approximately $15 \%$ confluence in separate $10 \mathrm{~cm}$ dishes for 72 hours. The $72-$ hour CM was then removed from all cell lines, centrifuged at 1,500 RPM for 10 minutes, and then the supernatants were collected, diluted 1:3 in fresh growth media, and applied to the differentiated C2C12 cells in Seahorse assay plates for 48 hours ( $n=10$ wells per treatment condition) prior to conducting the Agilent Seahorse XF Cell Mito Stress Test protocol according to manufacturer's instructions. 
In vitro CM PPAR-reporter assays. HEK293 cells were transfected with PPRE-H2beGFP 47(Addgene \#84393) using Invitrogen Lipofectamine 3000 (Thermo Fisher), selected with $500 \mathrm{ng} \cdot \mathrm{uL}-1$ Gibco geneticin (Thermo Fisher) for 20 days, and flow-sorted to select the cells expressing GFP. The resulting HEK293-PPRE-H2b-eGFP cell line was plated at approximately $15 \%$ confluence in a 24 -well plate. The following day, cells were imaged using the BioTek Cytation 5 Cell Imaging Multi-Mode Reader (Agilent Technologies) to collect baseline GFP intensity, and 72-hour conditioned media from HEK293, EpH4-EV, EO771, and NF639 cell lines was applied to the 24-well plate, using individual wells as biological replicates ( $n=6$ wells per treatment condition). Cells were then incubated in the conditioned media under normal culture conditions for 24 hours, at which point GFP intensity was measured using identical imaging settings as the baseline collection. Mean cellular GFP intensity per well was calculated using Gen5 Microplate Reader and Imager Software (Agilent Technologies) after background flattening and thresholding, which were set consistently across all images.

\section{Statistical Analyses.}

Clinical information: Patients' trends of weight change over time were calculated for each individual patient by fitting a simple linear regression line to their weight at each date in the EMR, normalized such that each patient's first weight record equaled 100, resulting in a slope representing their approximate percentage of body weight change per day. 19 patients were identified as outliers in terms of daily weight change. 13 of these patients were excluded due to having a limited observation period ( $<15$ days). Each patient's first albumin measurement was then obtained and the rate of daily weight change was regressed on the patients' first albumin measurements. Pearson's 
correlation coefficients and p-values were calculated and plotted using $g g p u b r{ }^{48}$ in $\mathrm{R}$ v3.6.1 $1^{49}$.

Logistic regression analysis was utilized to determine whether serum albumin was predictive for a rate of weight change consistent with cachexia (i.e. $<-0.027 \%$ per day to reach $5 \%$ weight loss in 6 months ${ }^{1,2}$ ). Omnibus model fit was assessed by Chisquare test and effect size was calculated using Nagelkerke's pseudo- $R^{2}$. The receiver operating characteristic curve (ROC) test was conducted, with preferred sensitivity and specificity $>0.7$. No point on the ROC satisfied these conditions. The fitted logistic regression model was used to predict whether each patient would exhibit a rate of weight loss consistent with cachexia, and these predictions were compared to the actual data to create a confusion matrix for determination of sensitivity, specificity, and positive predictive value.

RNA-Seq and clinical correlates: A multivariate regression model was used to assess clinical data against the 3D-MDS representing overall skeletal muscle gene expression, obtained as described above within "RNA-Sequencing." Each clinical variable available was regressed on the 3D-MDS and model fit was assessed via MANOVA and Pillai's trace. Forward selection was then applied to combine the variables with smallest $p$ values for Pillai's trace into a final model, with final selection considerations including statistical significance for MANCOVA using Pillai's trace and Wilks' lambda, maximizing Pillai's trace test statistic, and minimizing Wilks' lambda test statistic.

Differential gene expression analysis was conducted using DESeq $2^{35}$. Input data consisted of transcript-level abundance estimates from Salmon summarized to the gene level using tximport ${ }^{31}$. Two differential expression analyses were run: one comparing 
the group of $\mathrm{BC}$ patients to control patients, and another comparing the $\mathrm{BC}$ patients by subtype to control patients, with the null hypothesis rejected at FDR $<0.10$. Because molecular subtype was the only variable that yielded statistical significance for predicting gene expression in the multivariate regression model described above, no clinical characteristics were assessed in the differential expression analysis.

Proteomics: Differential protein expression analysis was conducted using $D E P^{50}$. Protein expression values were first filtered to remove known contaminants and proteins with missing expression values in more than 1 sample per group, then normalized, and then background-corrected using variance stabilizing transformation. Remaining missing values were imputed using k-nearest neighbor, after determining that the small number of missing values were likely missing at random. Differential expression analysis using linear models and empirical Bayes statistics was then conducted on the imputed dataset, with the null hypothesis rejected at FDR $<0.05$.

Enrichment analyses: Input data for GSEA included normalized gene expression counts for each patient for all genes with at least 1 CPM in at least 3 patients, with ranking conducted within GSEA using "Signal-to-noise" based ranking. Reference gene sets included all KEGG v7 pathways accessed from Molecular Signature Database ${ }^{51}$ with at least 15 genes in the pathway in our gene set for RNA-seq results and at least 10 genes in the pathway in our gene set for proteomics. Only pathways identified as enriched at q-value $<0.05$ are reported here. Input data for Enrichr ${ }^{52-53}$ analyses included only the list of significantly differentially expressed genes and proteins. In Enrichr, all databases were queried, with particular interest in ENCODE and ChEA Consensus TFs for transcription factor target enrichment, WikiPathways 2019 Human for pathway analysis, 
and 2018 databases from the Gene Ontology project for cellular component, biological process, and molecular function enrichment analyses. Data presented in this article reflect analyses conducted in Enrichr between May $15^{\text {th }}$ and June $15^{\text {th }}$ of 2019 and in GSEA between March $1^{\text {st }}$ and March $30^{\text {th }}$ 2020. Ingenuity Pathway Analysis (Qiagen, Germany) was utilized for visualization of affected pathways.

In vitro and in vivo validation assays: ATP content in each mitochondrial subpopulation in the PDOX muscle were compared to control using the Wilcoxon rank sum test. The null hypothesis was rejected at $p<0.05$. In the conditioned media metabolic experiments, one-way ANOVA was used to compare the rate of oxygen consumed in ATP production as a percentage of basal oxygen consumption between the four conditioned media treatment groups followed by two-tailed Student's t-tests with HolmBonferroni correction comparing each treatment group to CONMuscle. The null hypothesis was rejected at $p<0.05$. This experiment was conducted as reported twice with similar results, and results from the first analysis are reported. In the PPARresponsive reporter assays, mean GFP intensities in each well were normalized to account for differences in baseline GFP expression between wells. Normalized GFP intensities at 24 hours were compared to normalized baseline measurements using a paired samples two-tailed t-test by treatment group with Holm-Bonferroni correction for multiple comparisons. The null hypothesis was rejected at $p<0.05$. This experiment was conducted as reported twice with similar results, with results from the first analysis reported. This analysis includes $n=5$ for NF639-treated cells due to a technical problem during baseline image capture that resulted in the loss of one image. 
Chi-Square analyses: First, the actual number of unique and shared DEGs and dysregulated pathways were counted for each subtype. For example:

HER2-unique = genes differentially expressed only in HER2 patients HER2-2-Group $=$ HER2 $\cap$ ERPR $\quad+$ HER2 $\cap$ TP $\quad+$ HER2 $\cap$ TN; HER2-3-Group = HER2 $\cap$ ERPR $\cap$ TP + HER2 $\cap$ ERPR $\cap \mathrm{TN}+\mathrm{HER} 2 \cap \mathrm{TN} \cap$ TP. Then, expected numbers of DEGs and dysregulated pathways were calculated under the null hypothesis that DEGs and dysregulated pathways are independent of $B C$ subtype. Chi-square statistics were calculated using the difference between observed and expected numbers of DEGs and dysregulated pathways. On 6 degrees of freedom, the Chi-square critical value is 12.592 for statistical significance at $\alpha=0.05$. Bar plots represent the log-fold change in number of DEGs or dysregulated pathways compared to expected values, by category, and actual Chi-square statistics for each category are also provided.

Box-and-whisker plots: The width of the box represents the interquartile range (IQR) and whiskers extend to the single most extreme measurement in both directions, unless the most extreme measurement is considered an outlier, in which case the most extreme value is represented a dot without a connected whisker. Median values are represented by the horizontal line through each boxplot. Outliers in this context are defined as values more extreme than $1.5 \times \mathrm{IQR}$. 
DATA AVAILABILITY

Gene expression counts for RNA-seq and individual protein abundance estimates for proteomic data in human muscle biopsies have been provided for all patients (Supplemental Tables 1 and 2). At the time that we formulated our IRB Consent Form and started collecting muscle biopsies (December 2015), we did not have the foresight that the transcriptomic and proteomic data would need to be made available publicly in a more raw form. Our IRB Consent Form does not contain language pertaining to this, and therefore, the patients did not give consent to having their genetic information made publicly available. Reconsenting the patients in our study is logistically difficult, if not impossible, since some of our original patients may have completed treatment and are not visiting the Cancer Institute currently, or unfortunately may not have survived to this date. We acknowledge that this was an unfortunate error on our part.

\section{Article Preprint:}

This manuscript was previously published as a preprint at BioRxiv:

http://biorxiv.org/cgi/content/short/810952v1. Revisions have been made in response to peer review. 
FIGURES AND FIGURE LEGENDS

Figure 1: Skeletal Muscle Gene Expression Profiles.

A
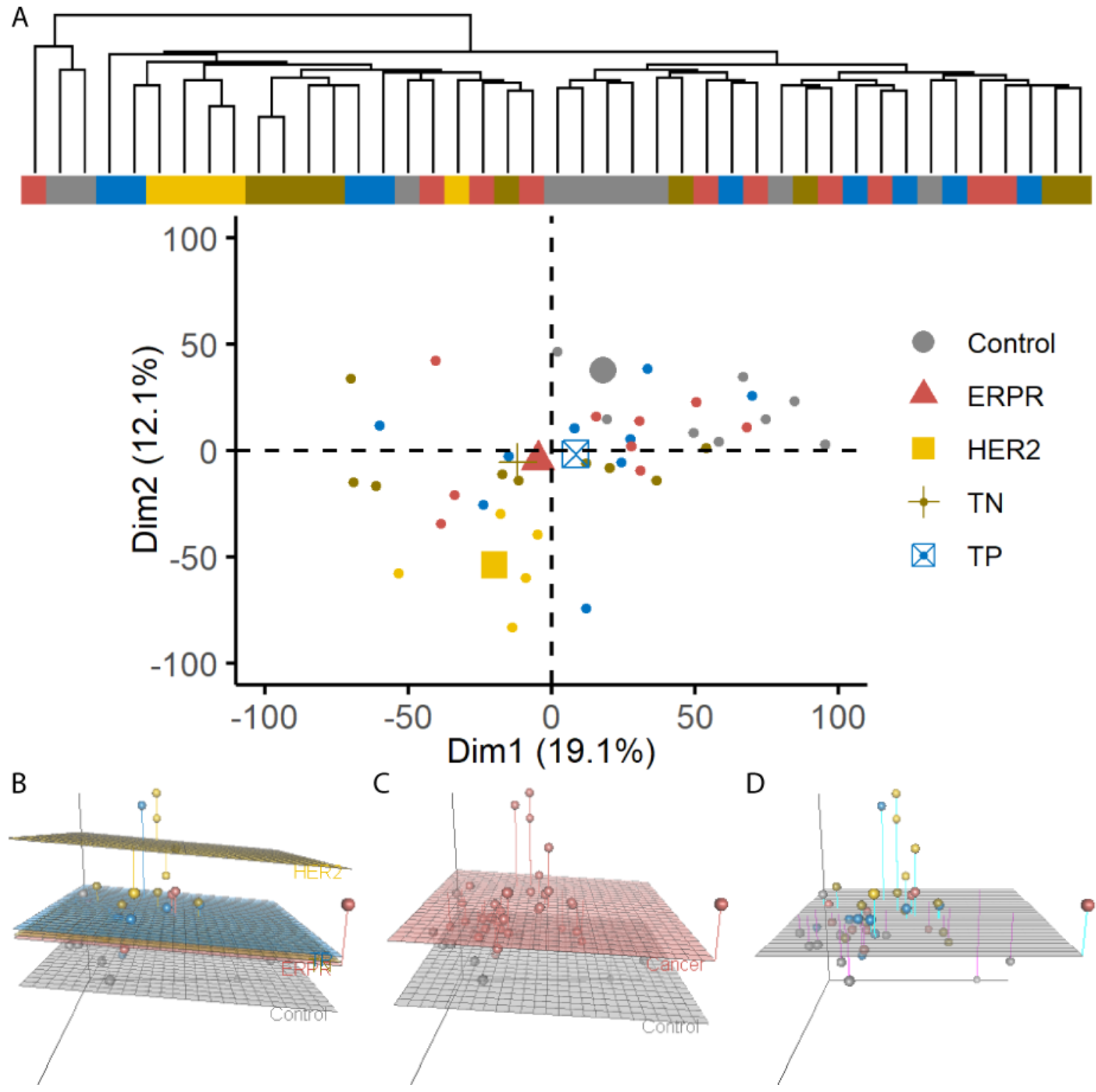

C
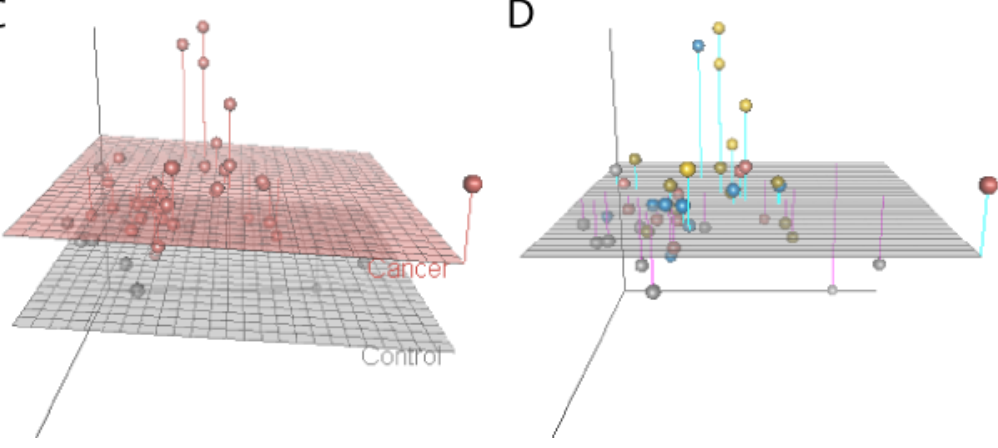

Unsupervised hierarchical clustering of individual patients on normalized, logtransformed RNA-seq gene expression data of the 10,000 most variable genes across all patients (A, top) and first two principal components calculated using filtered, normalized, log-transformed RNA-seq gene expression data with samples colored 
according to $\mathrm{BC}$ molecular subtype with group geometric means denoted with larger symbols (A, bottom). MDS dissimilarity matrix of overall gene expression data represented in 3 dimensions, with each dot representing an individual patient. Regression planes are color-coded based on molecular subtype (B), binary disease status (C), or the null model (D). 
Figure 2: Serum Albumin and Weight Loss.

A

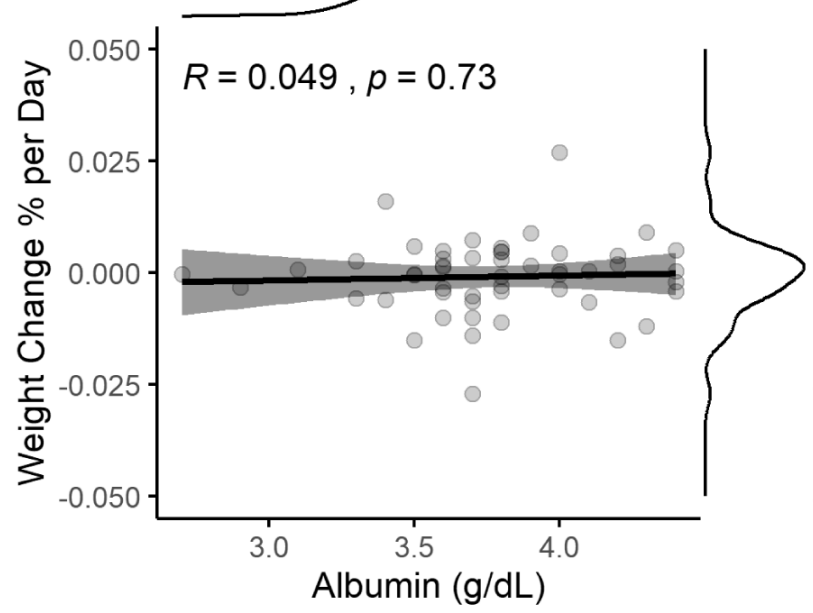

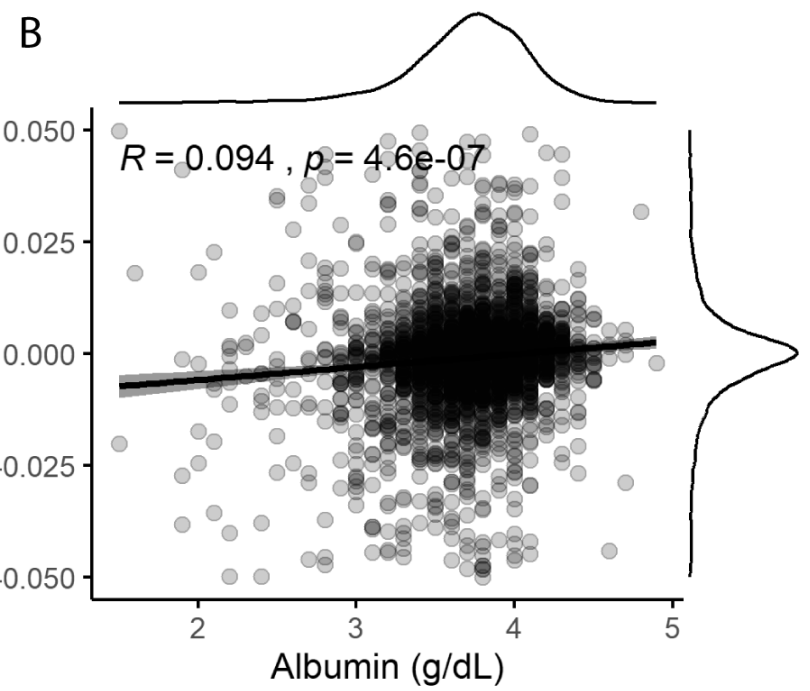

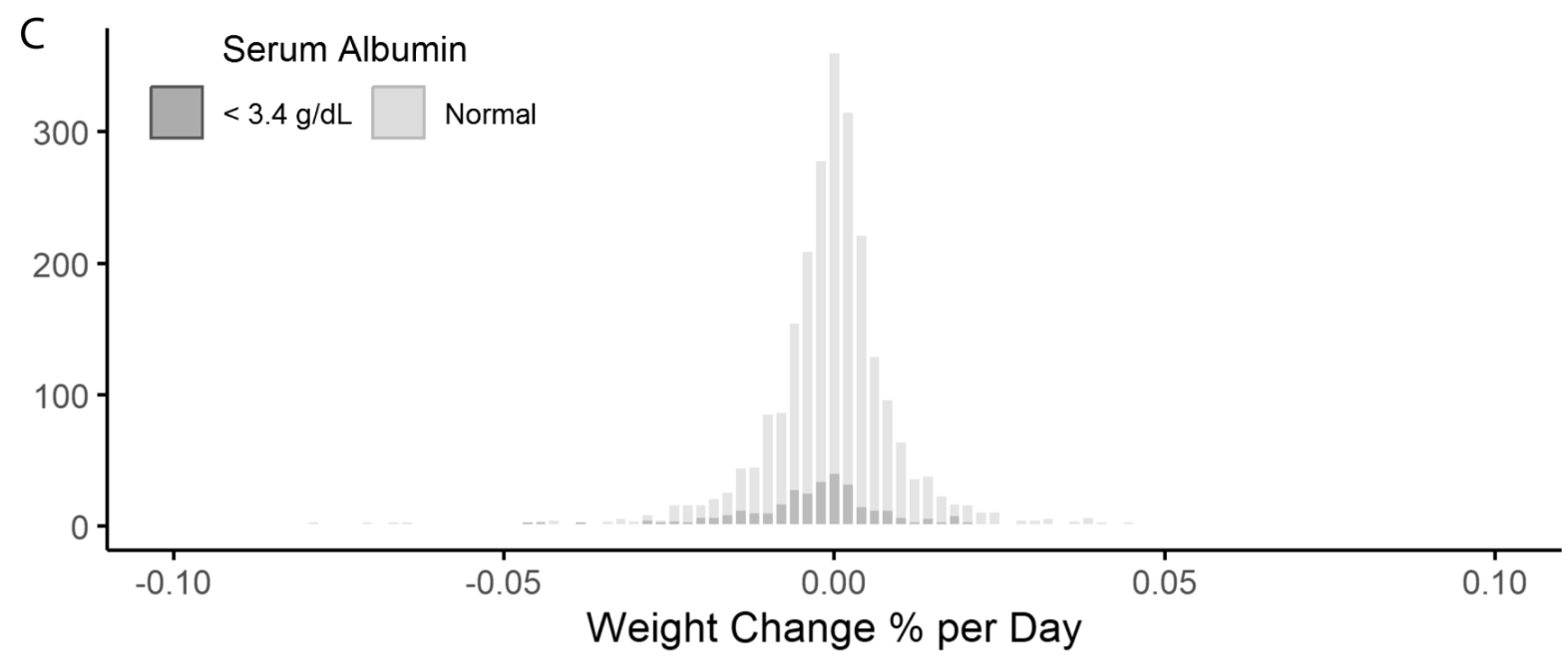

Linear regression analysis of trends in weight change predicted by serum albumin in patients with BC who provided muscle biopsies and at least one serum albumin measurement. Grey dots represent individual patients; grey shading represents $95 \%$ confidence interval of the black regression line, with marginal kernel density plots provided for both variables $(\mathbf{A}, \mathrm{n}=51)$. Linear regression analysis of trends in weight change predicted by serum albumin in a retrospective chart review of patients with BC. Grey dots represent individual patients; grey shading represents $95 \%$ confidence 
interval of the black regression line, with marginal kernel density plots provided for both variables $(B, n=3,001)$. Histogram representing individualized rate of weight change in a retrospective chart review of patients with $\mathrm{BC}$, with patients grouped by serum albumin level $\left(C, n_{\text {low }}=442, n_{\text {normal }}=2,557\right)$. 
Figure 3: Differential Gene Expression Analysis by Subtype.

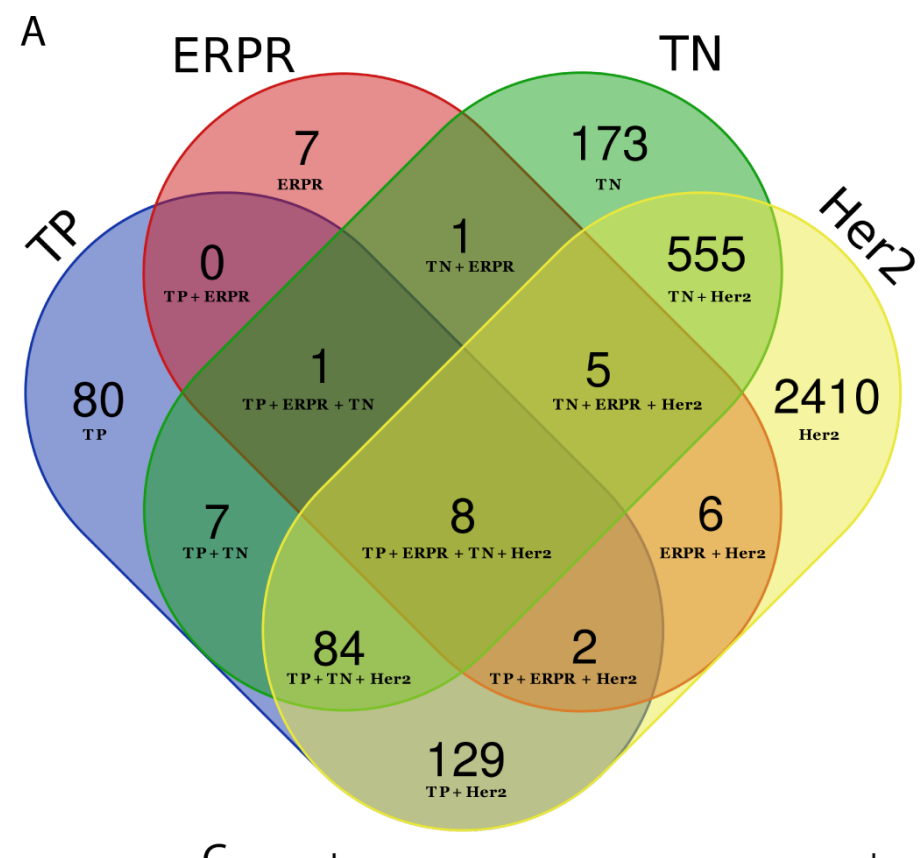

B
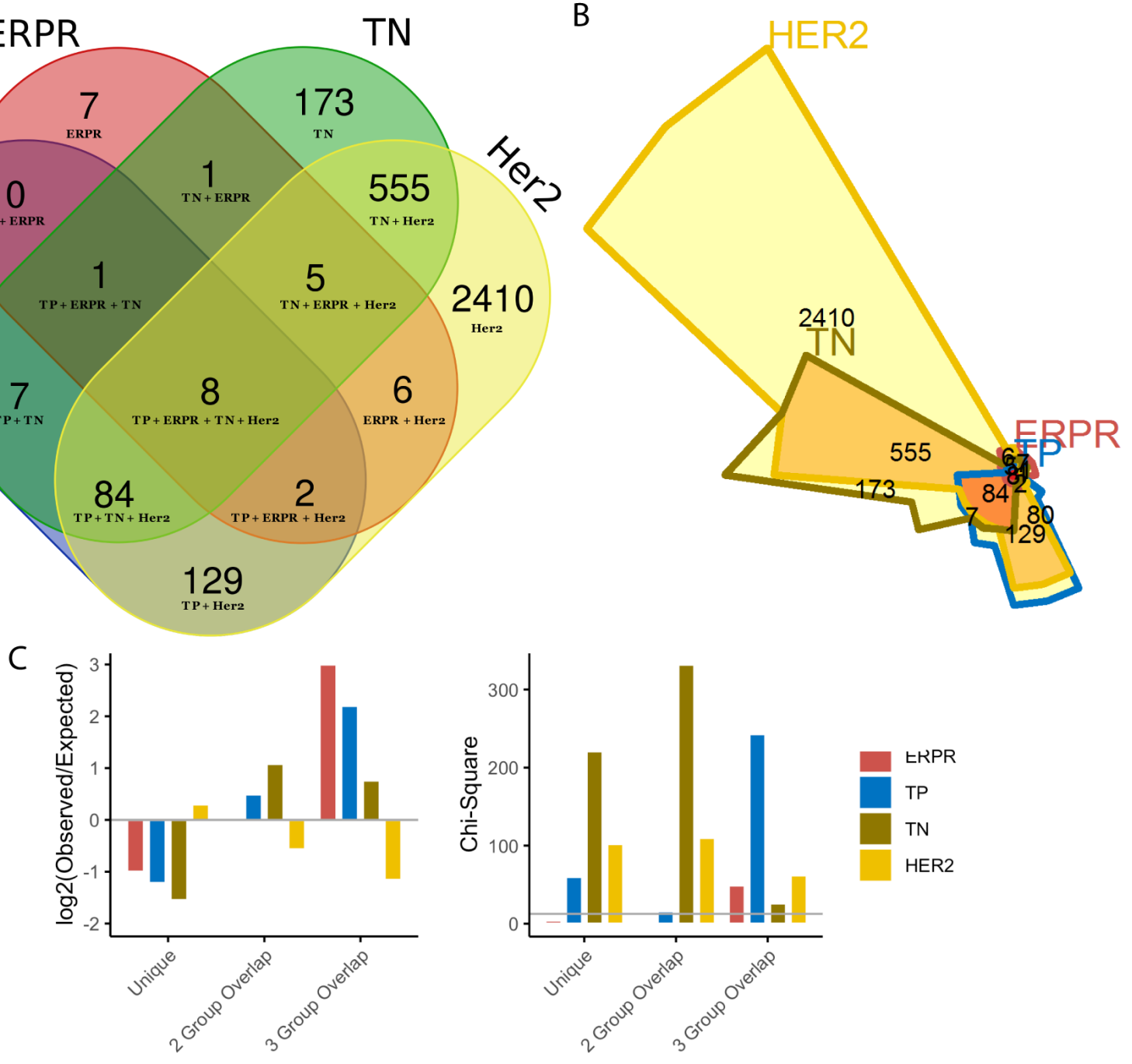

Venn diagram representing DEGs in muscle in each molecular subtype (A) and corresponding area-representative Chow-Ruskey diagram (B). Chi-square analysis of the number of DEGs uniquely differentially expressed in each subtype, the number of DEGs involved in 2- and 3-way overlaps among subtypes, showing the log-fold change between observed and expected numbers in each category. The Chi-square critical value of 12.592 ( 6 degrees of freedom) is denoted on the Chi-Square y-axis by a gray 
horizontal line. A Chi-square value larger than 12.592 is statistically significant at $\alpha=$ 0.05. (C). 
Figure 4: Dysregulated Pathway Analysis by Subtype.
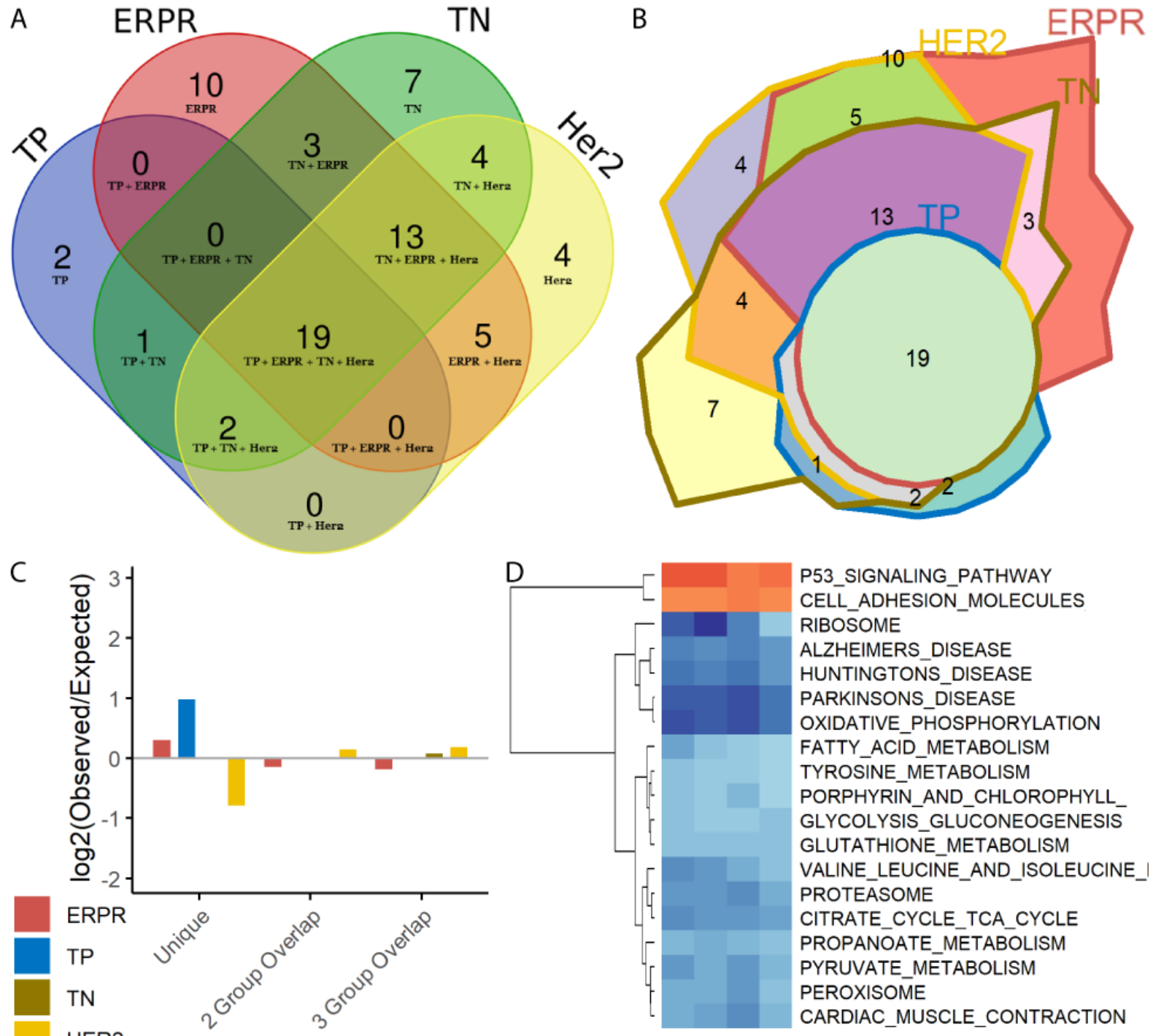

P53_SIGNALING_PATHWAY CELL_ADHESION_MOLECULES RIBOSOME

ALZHEIMERS_DISEASE HUNTINGTONS_DISEASE PARKINSONS_DISEASE OXIDATIVE PHOSPHORYLATION FATTY_ACID_METABOLISM TYROSINE_METABOLISM PORPHYRIN_AND_CHLOROPHYLL_ GLYCOLYSIS_GLUCONEOGENESIS GLUTATHIONE_METABOLISM VALINE_LEUCINE_AND_ISOLEUCINE_| PROTEASOME

CITRATE_CYCLE_TCA_CYCLE PROPANOATE_METABOLISM PYRUVATE_METABOLISM PEROXISOME CARDIAC_MUSCLE_CONTRACTION
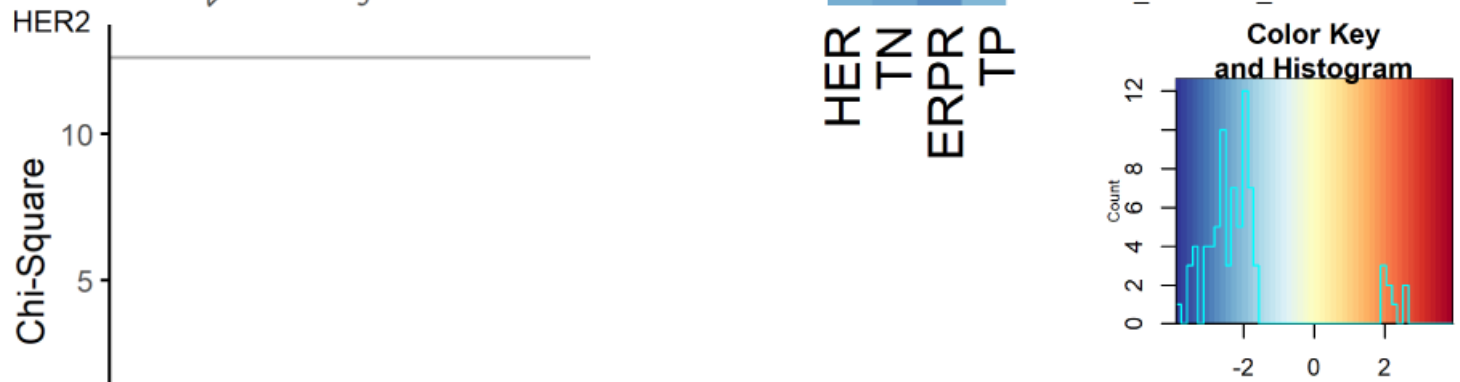
Venn diagram representing significantly dysregulated KEGG pathways in muscle in each molecular subtype (A) and corresponding area-representative Chow-Ruskey diagram (B). Chi-square analysis of the number of pathways uniquely dysregulated in each subtype and those involved in 2- and 3-way overlaps between subtypes, showing the log-fold change between observed and expected numbers in each category. The Chi-square critical value of 12.592 (6 degrees of freedom) is denoted on the Chi-square y-axis by a gray horizontal line. A Chi-square value larger than 12.592 is statistically significant at $\alpha=0.05$. (C). Heatmap of normalized enrichment scores from GSEA for the nineteen commonly dysregulated pathways across all subtypes, with the four columns of the heatmap representing the four molecular subtypes. Lower enrichment scores in blue indicate genes within that set are generally downregulated in the BC group, and higher enrichment scores in red indicate general upregulation in BC group relative to control (D). 
Figure 5: HER2 Patient Proteomics.

A

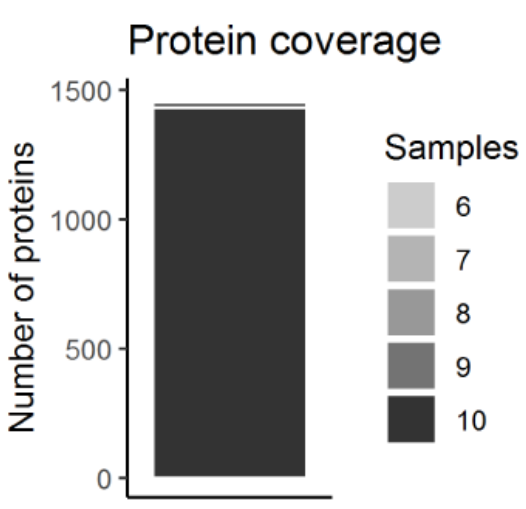

C

$\square$ Control $\square$ HER2
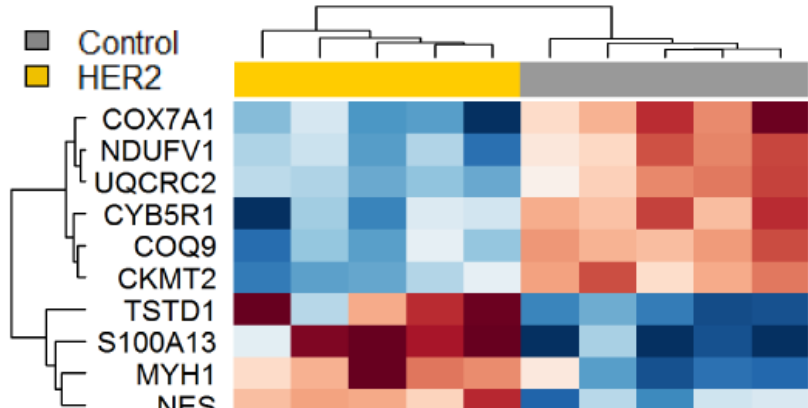

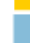

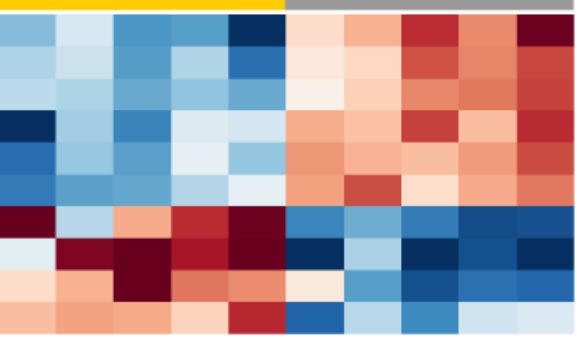

log2 Centered intensity

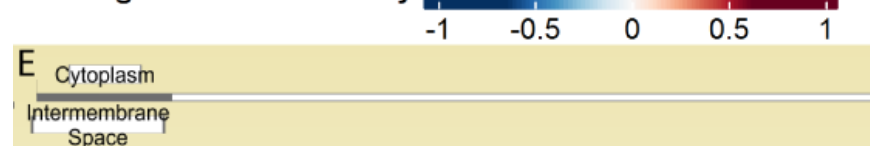

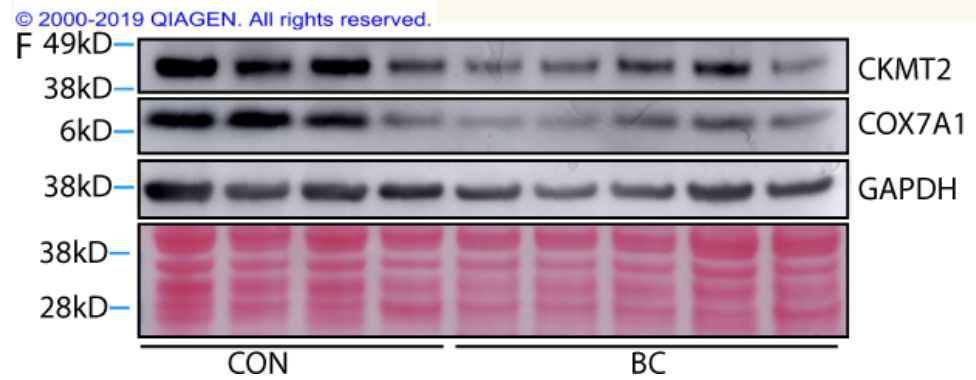

D
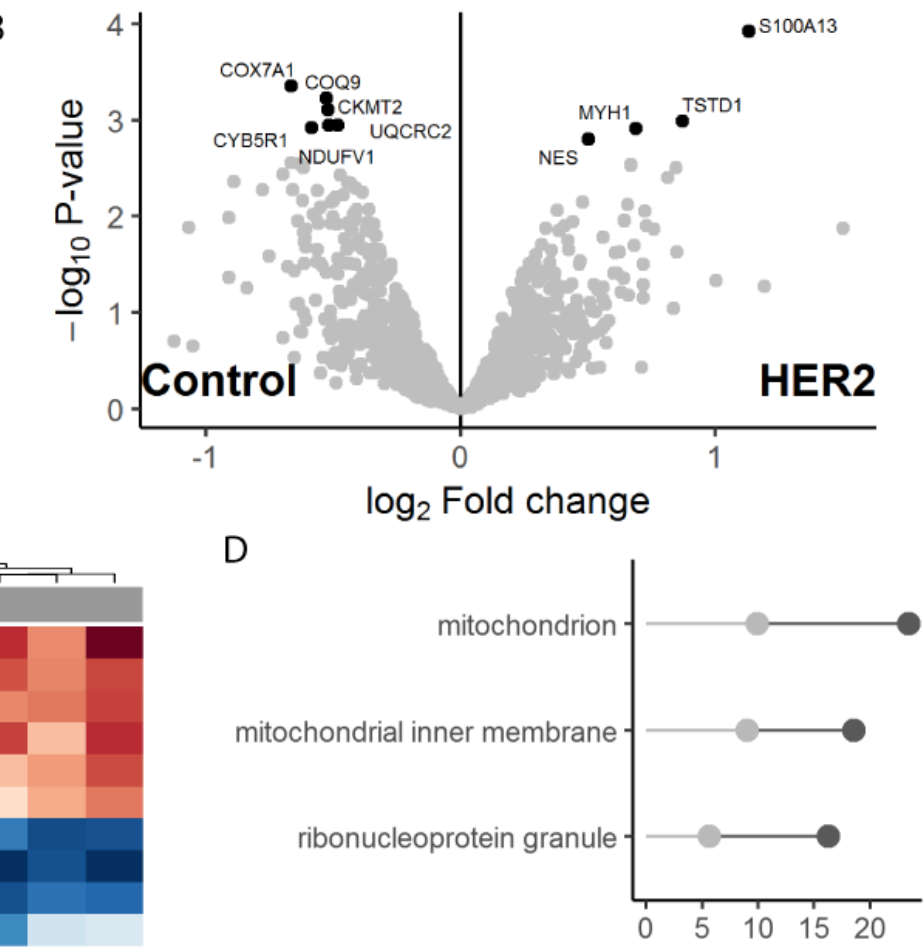

ф $-\log (p) \quad$ Enrichment Score
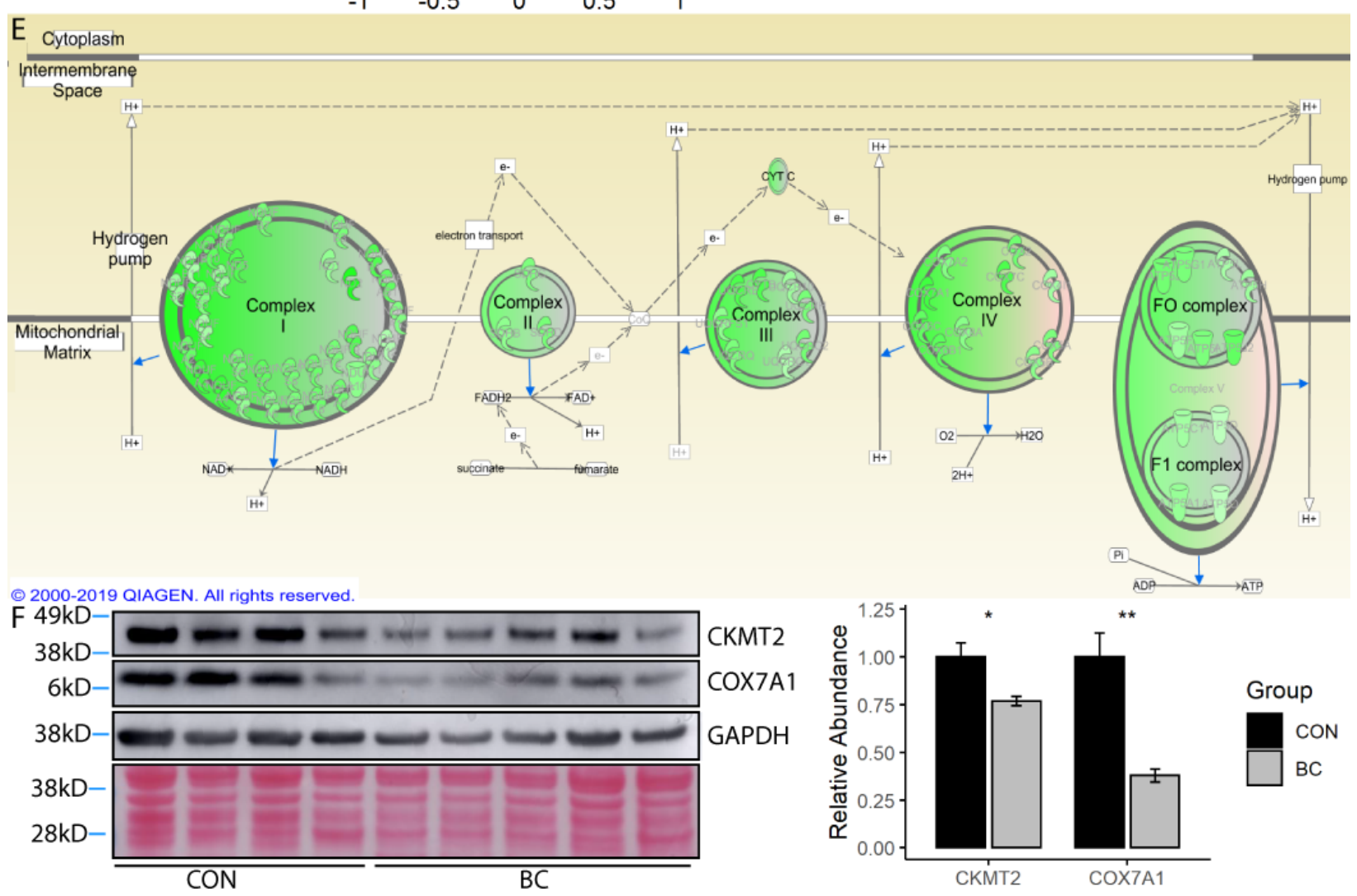
Stacked bar chart representing the number of unique proteins detected by the number of samples ( $\mathbf{A}, \mathrm{n}=10$ biopsies, $\mathrm{n}=1,555$ unique proteins). Differential expression analysis of detected proteins, represented as a volcano plot with significantly differentially expressed proteins (DEPs) identified in black (B). Heatmap of DEPs, with the ten columns representing the ten samples assayed (C). Enrichment analysis on the DEPs, querying Gene Ontology 2018 Cellular Components via Enrichr; top three results as ranked by Enrichment Score (D). Ingenuity Pathway Analysis representation of the mitochondrial electron transport chain, with proteins detected at lower abundance in the BC patient group notated in green (E). Western blot validation of two DEPs, using an independent cohort of BC and CON patients with Ponceau staining and GAPDH presented as loading controls $(\mathbf{F})$. 
Figure 6: Subtype-Independent DEG Analysis and Experimental Validation.
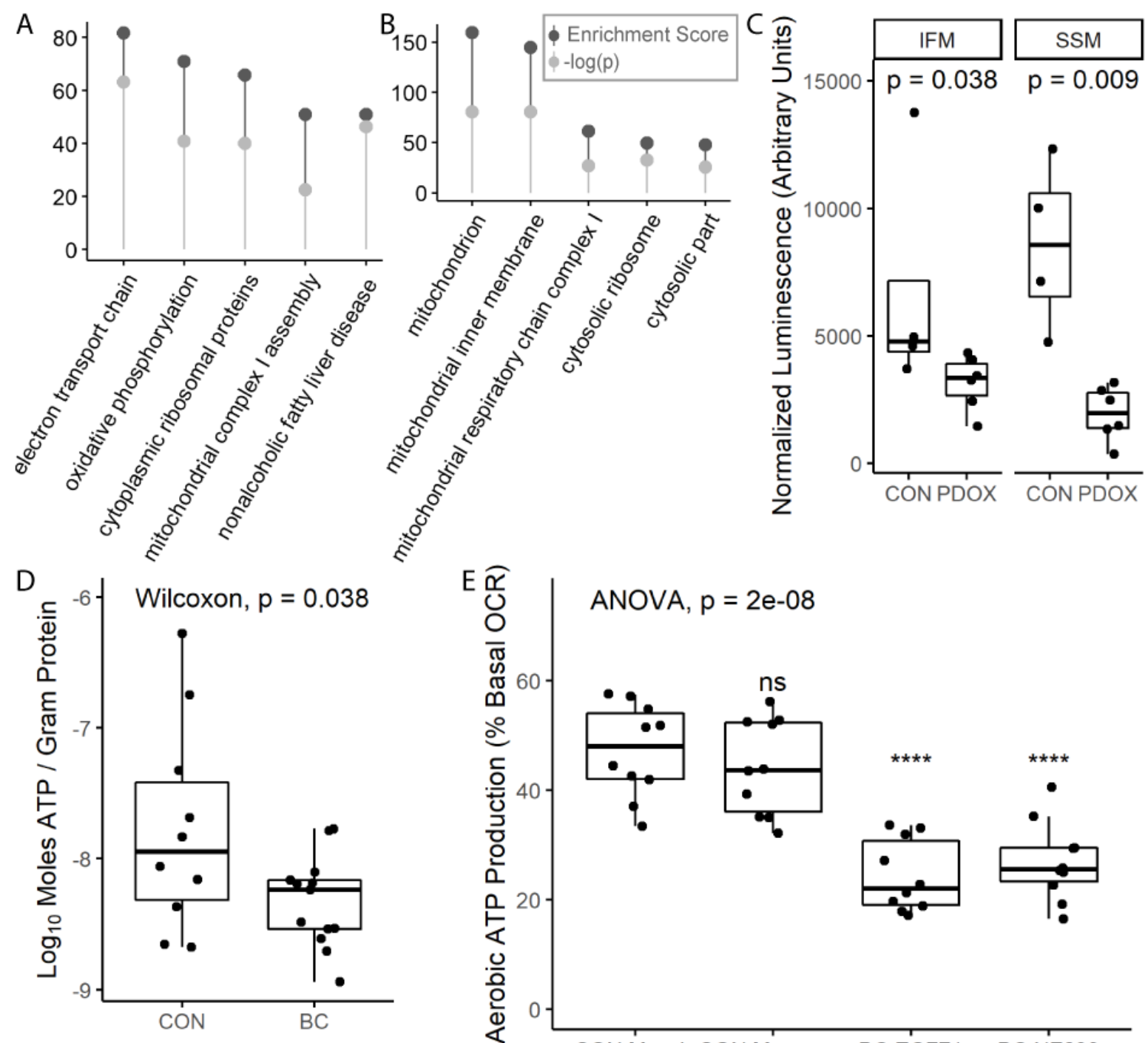

\begin{tabular}{l|l}
$E \widehat{\widetilde{r}}$ & ANOVA, $p=2 \mathrm{e}-08$
\end{tabular}
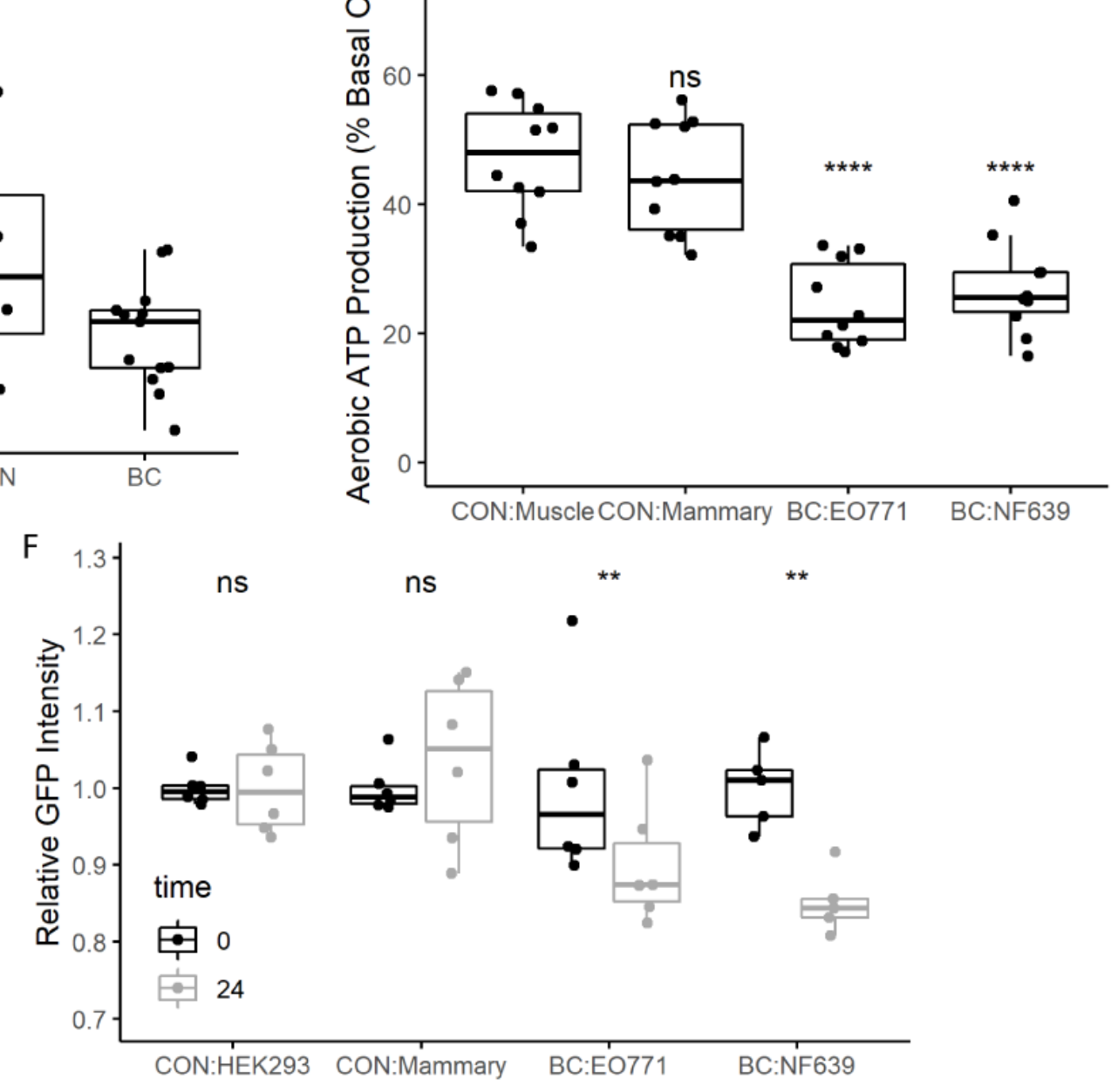
Enrichment analysis of DEGs, querying WikiPathways 2019 Human database. Top hits ranked by Enrichment Score (A). Enrichment analysis of DEGs, querying Gene Ontology 2018. Top hits ranked by Enrichment Score (B). Enrichment Score and pvalue reported as provided by Enrichr. A $-\log (\mathrm{p})>1.3$ corresponds to $p<0.05(\mathbf{A}, \mathbf{B})$. ATP content in quadriceps muscle of control $(n=4)$ and PDOX-bearing $(n=6)$ mice as assessed by chemiluminescence in two mitochondrial subpopulations. ATP content in the PDOX mitochondria was compared to control using the Wilcoxon rank sum test (C). ATP content in pectoralis major muscle of control $(n=10)$ and BC patients $(n=13)$ as assessed by chemiluminescence using a standard curve for absolute quantification and normalized to protein content. ATP content in the BC group was compared to control using the Wilcoxon rank sum test (D). Aerobic ATP production as a percentage of basal oxygen consumption rate $(\mathrm{OCR})$ in differentiated $\mathrm{C} 2 \mathrm{C} 12$ myotubes treated for 48 hours with media conditioned by C2C12 (CON-Muscle), EpH4-EV (CON-Mammary), EO771 (BC), or NF639 (BC) cells ( $n=10$ per group). Overall significance determined by oneway ANOVA followed by two-tailed Student's t-tests with Holm-Bonferroni correction comparing each treatment group to CON-Muscle (E). Normalized GFP intensity in HEK293-PPRE-H2b-eGFP reporter cells at baseline and after treatment with conditioned media for 24 hours ( $n=6$ for HEK293, EpH4-EV, and EO771; $n=5$ for NF639). Normalized GFP intensity values at 24 hours were compared to baseline measurements using paired samples two-tailed t-tests with Holm-Bonferroni correction of p-values (p. adj) (F). 


\section{Supplemental Figure 1.}
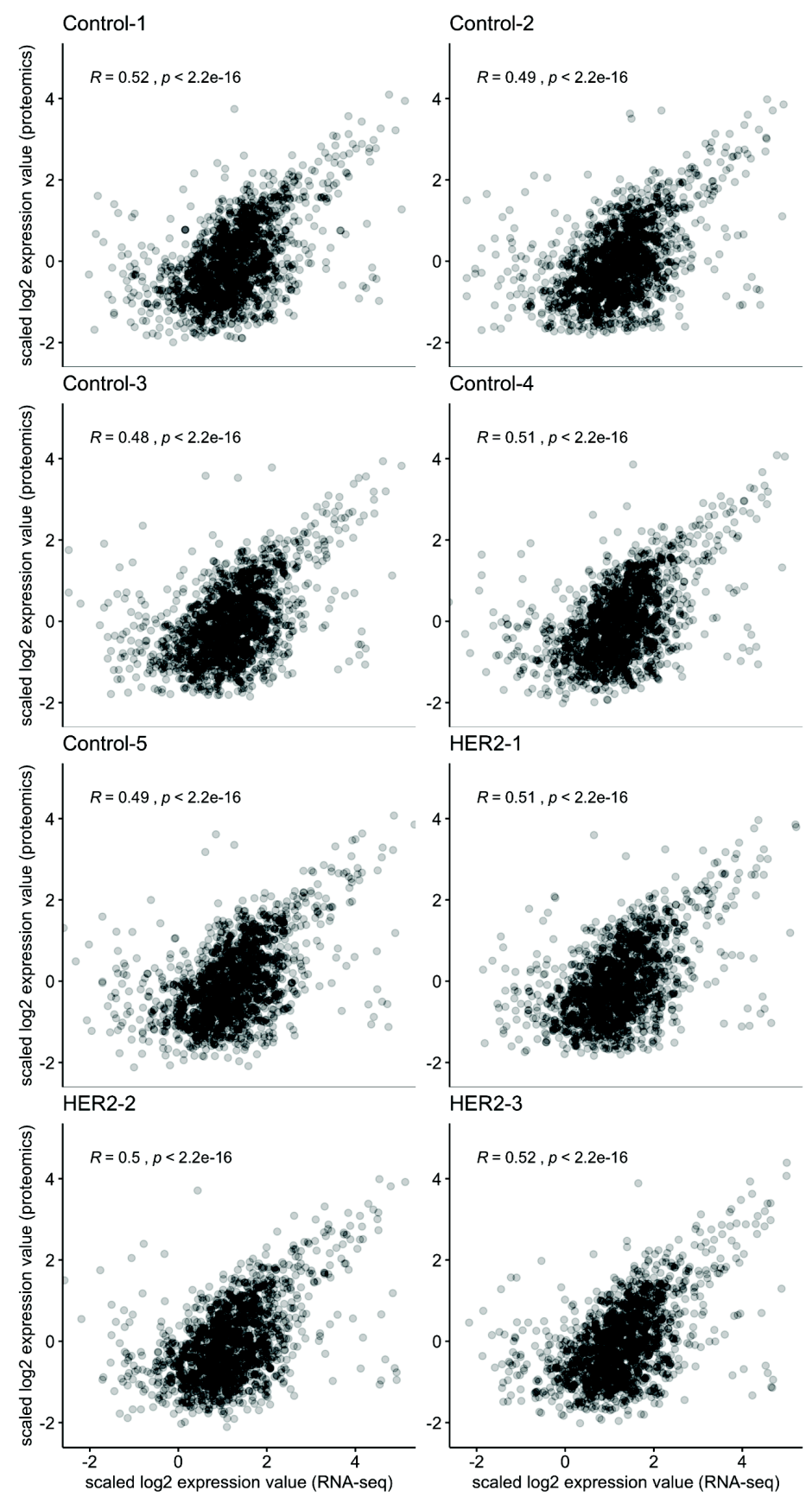

Scaled, log-transformed protein expression correlated with scaled, log-transformed RNA expression in a gene-wise fashion, presented for 8/8 patients with both RNAseq and proteomic analyses. 


\section{Supplemental Figure 2.}

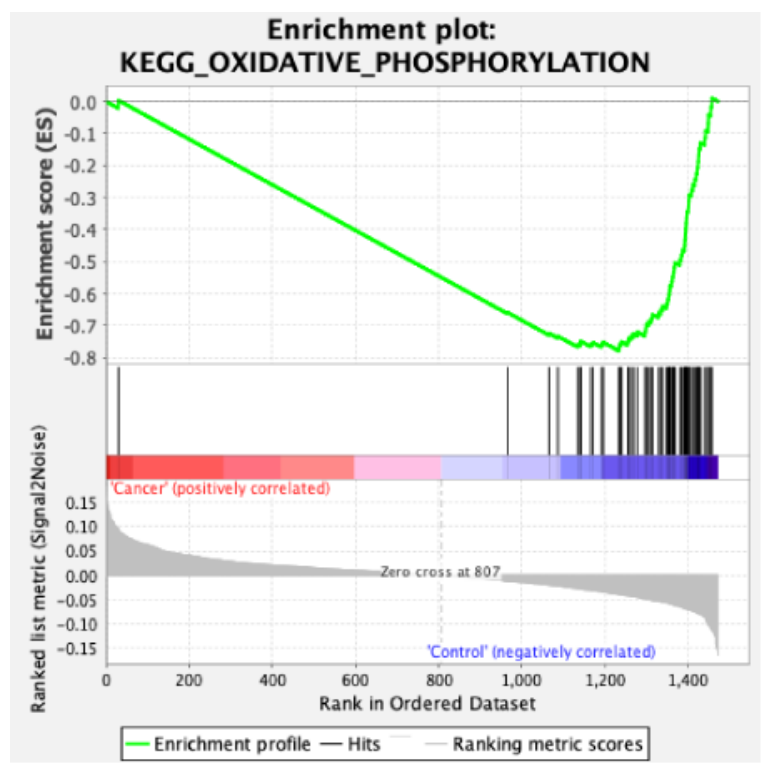

Enrichment plot: KEGG_CITRATE_CYCLE_TCA_CYCLE

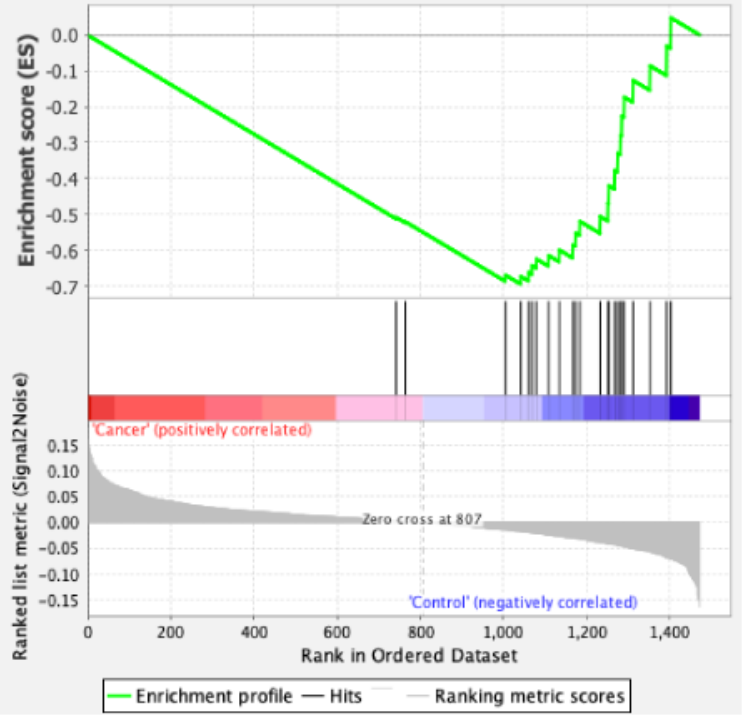

Enrichment plot: KEGG_PEROXISOME
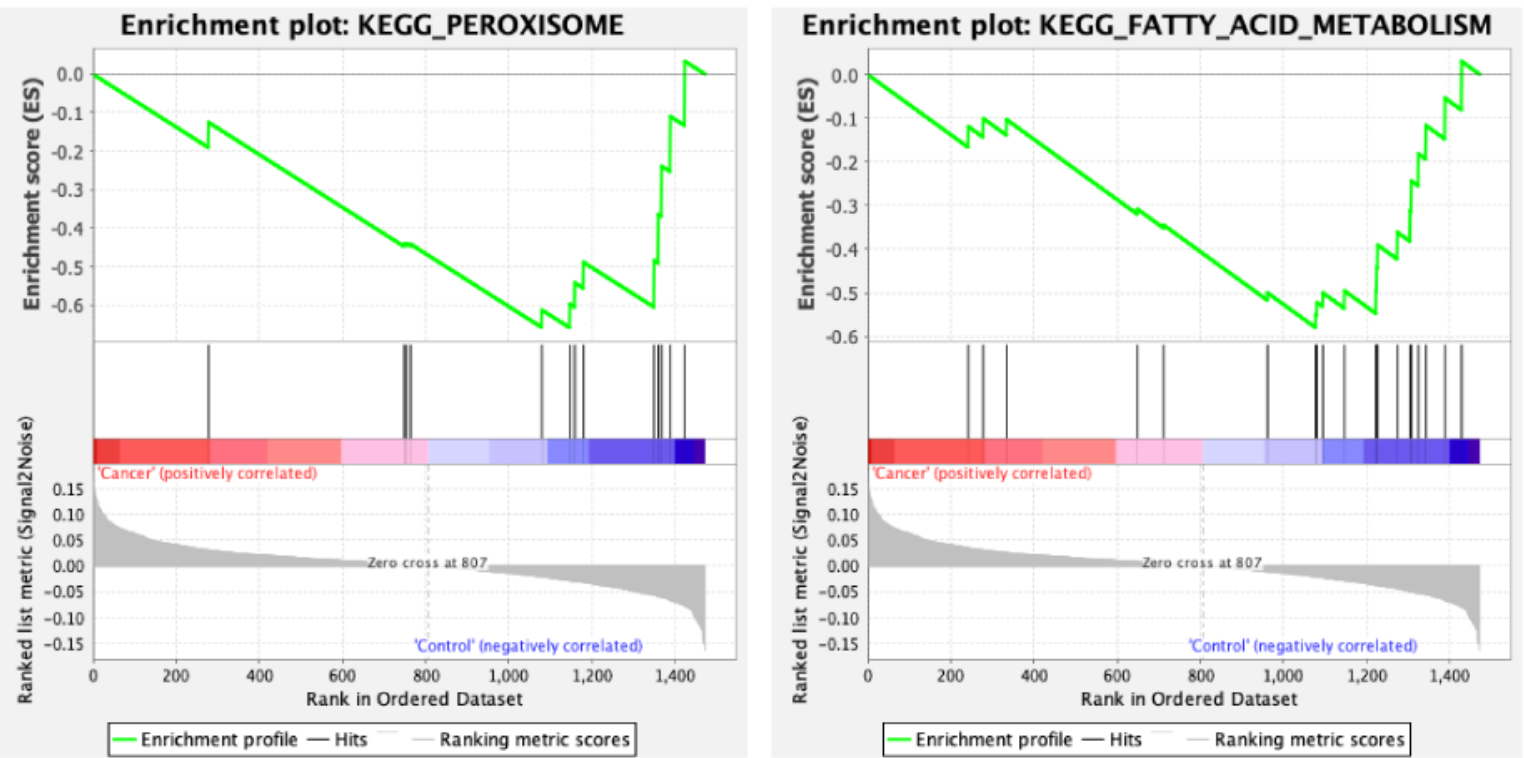

GSEA using proteomic data from muscle biopsies from $n=5$ women with HER2-

overexpressing tumors and $n=5$ control patients. 


\section{TABLES}

Table 1: Patient Characteristics

Patient Group BMI $\left(\mathrm{kgm}^{-2}\right)$ BMI Change Body Fat Lean Body Number of

(\%) (\%) Mass (kg) Days in Record

\begin{tabular}{r|l|l|l|l|l|}
\hline Control & $32.7 \pm 7.8$ & $-0.16 \pm 2.9$ & $37.7 \pm 7.5$ & $45.9 \pm 6.3$ & $220.1 \pm 135.1$ \\
\hline All Cancer & $29.9 \pm 7.4$ & $-0.32 \pm 5.10$ & $35.5 \pm 3.9$ & $46.4 \pm 5.6$ & $156.0 \pm 242.0$ \\
\hline ERPR & $28.6 \pm 4.5$ & $-0.54 \pm 2.9$ & & & $84.20 \pm 161.1$ \\
\hline HER2 & $31.1 \pm 7.8$ & $1.12 \pm 5.9$ & & & $159.9 \pm 131.9$ \\
\hline$T N$ & $29.6 \pm 6.5$ & $0.07 \pm 6.3$ & & & $313.5 \pm 426.6$ \\
\hline$T P$ & $31.6 \pm 11.6$ & $-1.47 \pm 6.5$ & & & $125.8 \pm 94.99$ \\
\hline$t$-Test, two-tailed p & $\mathrm{p}=0.18$ & $\mathrm{p}=0.90$ & $\mathrm{p}=0.24$ & $\mathrm{p}=0.82$ & $\mathrm{p}=0.27$ \\
$(D f, t)$ & $(69,1.36)$ & $(69,0.13)$ & $(41,1.19)$ & $(41,0.22)$ & $(69,1.12)$ \\
\hline ANOVA & $\mathrm{p}=0.70$ & $\mathrm{p}=0.72$ & & & $\mathrm{p}=0.08^{\circ}$ \\
$\left(D f_{\text {between }}\right.$, Df $\left.f_{\text {within }}, F\right)$ & $(3,47,0.47)$ & $(3,47,0.45)$ & & $(3,47,2.38)$ \\
\cline { 2 - 7 } & & & & & \\
\hline
\end{tabular}

All values are presented as mean \pm standard deviation; Df- degrees of freedom; ${ }^{\circ} \mathrm{p}<0.10$

t-Test: control vs. all cancer

ANOVA: among patient groups

$\mathrm{BMI}$ - body mass index; ER - estrogen receptor; $\mathrm{PR}$ - progesterone receptor 
Table 2: Possible clinical predictors of muscular gene expression

\begin{tabular}{|c|c|c|c|c|c|}
\hline Variable & Variable Type & Pillai's & Num & Den & $\mathbf{p}$ \\
\hline & (Levels or Range) & Trace & Df & Df & \\
\hline Group & $\begin{array}{l}\text { Factor } \\
\text { (Control, ERPR, HER2, TN,TP) }\end{array}$ & 0.51 & 12 & 111 & $0.04 *$ \\
\hline Serum Albumin & Numeric & 0.20 & 3 & 29 & $0.08^{\circ}$ \\
\hline N Staging & Factor $(N 0, N 1, N 1 a, N 2, N 3)$ & 0.52 & 12 & 84 & 0.15 \\
\hline \# of Chemotherapy Txs & Numeric $(0-2)$ & 0.10 & 3 & 38 & 0.25 \\
\hline \# of Radiation Txs & Numeric $(0-1)$ & 0.10 & 3 & 38 & 0.26 \\
\hline T Staging & Factor $(T 1, T 1 a, T 2, T 2 A, T 3, T 4)$ & 0.51 & 15 & 81 & 0.37 \\
\hline \# of Hormonal Txs & Numeric $(0-1)$ & 0.07 & 3 & 38 & 0.43 \\
\hline$L B M \%$ & Numeric & 0.12 & 3 & 19 & 0.45 \\
\hline$B M I$ & Numeric & 0.05 & 3 & 38 & 0.56 \\
\hline Ave. Daily BMI Change & Numeric & 0.03 & 3 & 37 & 0.78 \\
\hline M Staging & Factor $(M O, M X)$ & 0.01 & 3 & 29 & 0.95 \\
\hline
\end{tabular}

Num- numerator; Den- denominator; Df- degrees of freedom; LBM \%- Lean body mass as a percentage of total body mass; Ave- Average; $\mathrm{T}$ - tumor; $\mathrm{N}$ - lymph node; $\mathrm{M}$ - metastatic; Txscourses of treatments; $* \mathrm{p}<0.05,{ }^{\circ} \mathrm{p}<0.10$ 
Table 3: Final clinical predictors of muscular gene expression

\begin{tabular}{|c|c|c|c|}
\hline Model Formula & $\begin{array}{l}\text { F-Statistics for Model Fit, } \\
\text { By Dimension }\end{array}$ & $\begin{array}{l}\text { Pillai's Trace } \\
\text { (p-value) }\end{array}$ & $\begin{array}{l}\text { Wilk's Lambda } \\
\text { (p-value) }\end{array}$ \\
\hline$(D 1, D 2, D 3) \sim$ Patient Group & $\begin{array}{l}\text { D1: } 0.3 \\
\text { D2: } 6.9 \\
\text { D3: } 0.4\end{array}$ & $\begin{array}{l}0.506 \\
(0.044) *\end{array}$ & $\begin{array}{l}0.522 \\
(0.020) *\end{array}$ \\
\hline $\begin{array}{r}(D 1, D 2, D 3) \sim \text { Patient Group } \\
+ \text { Serum Albumin }\end{array}$ & $\begin{array}{l}\text { D1: } 0.7 \\
\text { D2: } 3.5 \\
\text { D3: } 2.1\end{array}$ & $\begin{array}{l}0.744 \\
(0.052)^{\mathrm{o}}\end{array}$ & $\begin{array}{l}0.401 \\
(0.049) *\end{array}$ \\
\hline
\end{tabular}

D1-D3- individual dimensions of RNA-sequencing data as 3-dimensional dissimilarity matrix; $* \mathrm{p}<0.05,{ }^{\circ} \mathrm{p}<0.10$ 


\section{REFERENCES}

1. Fearon, K., et al. Definition and classification of cancer cachexia: an international consensus. Lancet Oncol 12, 489-495 (2011).

2. Evans, W.J., et al. Cachexia: a new definition. Clin Nutr 27, 793-799 (2008).

3. Kilgour, R.D., et al. Cancer-related fatigue: the impact of skeletal muscle mass and strength in patients with advanced cancer. J Cachexia Sarcopenia Muscle 1, $177-185$ (2010).

4. Neefjes, E.C.W., et al. Muscle mass as a target to reduce fatigue in patients with advanced cancer. J Cachexia Sarcopenia Muscle 8, 623-629 (2017).

5. Stephens, N.A., et al. Sexual dimorphism modulates the impact of cancer cachexia on lower limb muscle mass and function. Clin Nutr 31, 499-505 (2012).

6. Arndt, V., Stegmaier, C., Ziegler, H. \& Brenner, H. A population-based study of the impact of specific symptoms on quality of life in women with breast cancer 1 year after diagnosis. Cancer 107, 2496-2503 (2006).

7. Peters, K.B., et al. Impact of health-related quality of life and fatigue on survival of recurrent high-grade glioma patients. J Neurooncol 120, 499-506 (2014).

8. Wang, X.S. \& Woodruff, J.F. Cancer-related and treatment-related fatigue. Gynecol Oncol 136, 446-452 (2015).

9. Groenvold, M., et al. Psychological distress and fatigue predicted recurrence and survival in primary breast cancer patients. Breast Cancer Res Treat 105, 209-219 (2007). 
10. Prado, C.M., et al. Sarcopenia as a determinant of chemotherapy toxicity and time to tumor progression in metastatic breast cancer patients receiving capecitabine treatment. Clin Cancer Res 15, 2920-2926 (2009).

11. Curt, G.A., et al. Impact of cancer-related fatigue on the lives of patients: new findings from the Fatigue Coalition. Oncologist 5, 353-360 (2000).

12. Bower, J.E. Cancer-related fatigue--mechanisms, risk factors, and treatments. Nat Rev Clin Oncol 11, 597-609 (2014).

13. Escalante, C. Cancer-related fatigue: Treatment - UpToDate. Vol. 2019 (UpToDate, 2019).

14. Bohlen, J., et al. Dysregulation of metabolic-associated pathways in muscle of breast cancer patients: preclinical evaluation of interleukin-15 targeting fatigue. $J$ Cachexia Sarcopenia Muscle (2018).

15. Wilson, H.E., et al. Human Breast Cancer Xenograft Model Implicates Peroxisome Proliferator-activated Receptor Signaling as Driver of Cancerinduced Muscle Fatigue. Clin Cancer Res 25, 2336-2347 (2019).

16. Subramanian, A., Kuehn, H., Gould, J. et.al. GSEA-P: a desktop application for Gene Set Enrichment Analysis. Bioinformatics 23, 23, 3251-3252 (2007).

17. Kanehisa, M., Sato, Y., Furumichi, M., Morishima, K. \& Tanabe, M. New approach for understanding genome variations in KEGG. Nucleic Acids Res 47, D590-d595 (2019).

18. Larsen, S., et al. Biomarkers of mitochondrial content in skeletal muscle of healthy young human subjects. J Physiol 590, 3349-3360 (2012). 
19. Wu, Z., et al. Mechanisms controlling mitochondrial biogenesis and respiration through the thermogenic coactivator PGC-1. Cell 98, 115-124 (1999).

20. Adhihetty, P.J., et al. The role of PGC-1alpha on mitochondrial function and apoptotic susceptibility in muscle. Am J Physiol Cell Physiol 297, C217-225 (2009).

21. St-Pierre, J., et al. Suppression of reactive oxygen species and neurodegeneration by the PGC-1 transcriptional coactivators. Cell 127, 397-408 (2006).

22. Calvo, J.A., et al. Muscle-specific expression of PPARgamma coactivator-1alpha improves exercise performance and increases peak oxygen uptake. J Appl Physiol (1985) 104, 1304-1312 (2008).

23. Olefsky, J.M. \& Saltiel, A.R. PPAR gamma and the treatment of insulin resistance. Trends Endocrinol Metab 11, 362-368 (2000).

24. Lipscombe, L.L., et al. Incidence of diabetes among postmenopausal breast cancer survivors. Diabetologia 56, 476-483 (2013).

25. Orgel, E. \& Mittelman, S.D. The Links Between Insulin Resistance, Diabetes, and Cancer. Curr Diab Rep 13, 213-222 (2013).

26. McMillan, D.C. Systemic inflammation, nutritional status and survival in patients with cancer. Curr Opin Clin Nutr Metab Care 12, 223-226 (2009).

27. Sullivan, D.H. What do the serum proteins tell us about our elderly patients? $J$ Gerontol A Biol Sci Med Sci 56, M71-74 (2001). 
28. Onate-Ocana, L.F., et al. Serum albumin as a significant prognostic factor for patients with gastric carcinoma. Ann Surg Oncol 14, 381-389 (2007).

29. Lambert, J.W., et al. Using preoperative albumin levels as a surrogate marker for outcomes after radical cystectomy for bladder cancer. Urology 81, 587-592 (2013).

30. Schalk, B.W., Deeg, D.J., Penninx, B.W., Bouter, L.M. \& Visser, M. Serum albumin and muscle strength: a longitudinal study in older men and women. J Am Geriatr Soc 53, 1331-1338 (2005).

31. Bae, J.C., et al. Association between Serum Albumin, Insulin Resistance, and Incident Diabetes in Nondiabetic Subjects. Endocrinol Metab (Seoul) 28, 26-32 (2013).

32. Patro, R., Duggal, G., Love, M.I., Irizarry, R.A. \& Kingsford, C. Salmon provides fast and bias-aware quantification of transcript expression. Nat Methods 14, 417419 (2017).

33. Soneson, C., Love, M.I. \& Robinson, M.D. Differential analyses for RNA-seq: transcriptlevel estimates improve gene-level inferences. F1000Res 4, 1521 (2015).

34. Robinson, M.D., McCarthy, D.J. \& Smyth, G.K. edgeR: a Bioconductor package for differential expression analysis of digital gene expression data. Bioinformatics 26, 139140 (2010).

35. Warnes, G., et al. gplots: Various R Programming Tools for Plotting Data. (CRAN (Comprehensive R Archive Network), 2019). 
36. Fox, J. \& Weisberg, S. car: Companion to Applied Regression. in Comprehensive R Archive Network (CRAN) (2019).

37. Love, M.I., Huber, W. \& Anders, S. Moderated estimation of fold change and dispersion for RNA-seq data with DESeq2. Genome Biol 15, 550 (2014).

38. Zhao, S., Li, C., Guo, Y. et. al. RnaSeqSampleSize: real data based sample size estimation for RNA sequencing. BMC Bioinformatics 19, 191 (2018). https://doi.org/10.1186/s12859-018-2191-5.

39. Bae, J., Kim, S., Lee, S. et.al. Comprehensive proteome and phosphoproteome profiling shows negligible influence of RNAlater on protein abundance and phosphorylation. Clin Proteom 16, 18 (2019). https://doi.org/10.1186/s12014019-9239-z.

40. Butovsky, O., et al. Identification of a unique TGF-beta-dependent molecular and functional signature in microglia. Nat Neurosci 17, 131-143 (2014).

41. Butovsky, O., et al. Targeting miR-155 restores abnormal microglia and attenuates disease in SOD1 mice. Ann Neurol 77, 75-99 (2015).

42. Kall, L., Storey, J.D. \& Noble, W.S. Non-parametric estimation of posterior error probabilities associated with peptides identified by tandem mass spectrometry. Bioinformatics 24, i42-48 (2008).

43. Palmer, J.W., Tandler, B. \& Hoppel, C.L. Biochemical properties of subsarcolemmal and interfibrillar mitochondria isolated from rat cardiac muscle. $J$ Biol Chem 252, 8731-8739 (1977). 
44. Dabkowski, E.R., Williamson, C.L. \& Hollander, J.M. Mitochondria-specific transgenic overexpression of phospholipid hydroperoxide glutathione peroxidase (GPx4) attenuates ischemia/reperfusion-associated cardiac dysfunction. Free Radic Biol Med 45, 855-865 (2008).

45. Dabkowski, E.R., et al. Mitochondrial dysfunction in the type 2 diabetic heart is associated with alterations in spatially distinct mitochondrial proteomes. $A m \mathrm{~J}$ Physiol Heart Circ Physiol 299, H529-540 (2010).

46. O'Connell, G.C., et al. IL-15Ra deficiency in skeletal muscle alters respiratory function and the proteome of mitochondrial subpopulations independent of changes to the mitochondrial genome. Mitochondrion 25, 87-97 (2015).

47. Degrelle, S.A., Shoaito, H. \& Fournier, T. New Transcriptional Reporters to Quantify and Monitor PPARgamma Activity. PPAR Res 2017, 6139107 (2017).

48. Kassambara, A. ggpubr: 'ggplot2' Based Publication Ready Plots. in Comprehensive R Archive Network (CRAN) (Comprehensive R Archive Network (CRAN), 2019).

49. R Core Team. R: A language and environment for statistical computing. ( $R$ Foundation for Statistical Computing, Vienna, Austria, 2019).

50. Zhang, X., et al. Proteome-wide identification of ubiquitin interactions using UbIAMS. Nat Protoc 13, 530-550 (2018).

51. Liberzon, A. et al. Molecular signatures database (MSigDB) 3.0. Bioinformatics 27, $1739-40$ (2011). 
52. Kuleshov, M.V., et al. Enrichr: a comprehensive gene set enrichment analysis web server 2016 update. Nucleic Acids Res 44, W90-97 (2016).

53. Chen, E.Y., et al. Enrichr: interactive and collaborative HTML5 gene list enrichment analysis tool. in BMC Bioinformatics, Vol. 14128 (2013). 
CHAPTER 4: Causal mediation analysis of muscle wasting on the muscle fatigue response to experimentally-induced breast cancer

A commonly accepted dogma in the field of cancer cachexia research is the assumption that the muscle dysfunction that occurs in cancer patients is entirely a results of muscle atrophy. This article utilized the statistical technique of causal mediation analysis to determine whether the increased fatigability exhibited in two mouse models of $B C$ is mediated by muscle atrophy. These models included the PDOX model, which exhibits increased fatigability in the absence of muscle wasting, and the EO771 orthotopic syngeneic model, which exhibits increased fatigability in all mice but with varying degrees of atrophy. Here, we show that muscle atrophy is likely not a mediator of the increased fatigability exhibited in these mouse models.

In this project, I independently carried out the statistical analysis, made several of the figures, and significantly contributed to the manuscript text.

This article is currently under review at PLOS ONE as of April 24, 2020. 


\section{Causal mediation analysis of muscle wasting on the muscle fatigue response to experimentally-induced breast cancer}

Hannah E. Wilson ${ }^{1,2}$, Emidio E. Pistilli ${ }^{* 2,3,4,5}$, David A. Stanton ${ }^{3}$, Joseph Bohlen ${ }^{6}$, Elena N. Pugacheva ${ }^{2,7}$, Sijin Wen ${ }^{2,8}$, and Sarah L. McLaughlin ${ }^{2,9}$.

${ }^{5} \mathrm{MD} / \mathrm{PhD}$ Medical Scientist Program, West Virginia University School of Medicine, Morgantown, WV, 26506.

${ }^{2}$ Cancer Institute, West Virginia University School of Medicine, Morgantown, WV, 26506.

${ }^{1}$ Division of Exercise Physiology, Department of Human Performance, West Virginia University School of Medicine, Morgantown, WV, 26506.

${ }^{3}$ Department of Microbiology, Immunology and Cell Biology, West Virginia University School of Medicine, Morgantown, WV, 26506.

${ }^{4}$ West Virginia Clinical and Translational Sciences Institute, West Virginia University School of Medicine, Morgantown, WV, 26506.

${ }^{6}$ Lieber Institute for Brain Development, Johns Hopkins Medical School, Baltimore, MD, 21205.

${ }^{7}$ Department of Biochemistry, West Virginia University School of Medicine, Morgantown, WV, 26506.

${ }^{8}$ Department of Biostatistics, West Virginia University School of Public Health, Morgantown, WV, 26506.

${ }^{9}$ Animal Models and Imaging Facility, West Virginia University School of Medicine, Morgantown, WV, 26506.

${ }^{*}$ Corresponding Author:

Emidio E. Pistilli, PhD

West Virginia University School of Medicine

Morgantown, WV 26506

304-293-0291 (office)

304-293-7105 (fax)

epistilli2@hsc.wvu.edu

Running title: Muscle fatigue and experimentally-induced breast cancer

Keywords: fatigue, muscle wasting, cachexia, breast cancer 


\section{ABSTRACT}

Background: Skeletal muscle fatigue is often considered to be linked to muscle wasting in patients with cancer and in experimental models of cancer. The purpose of this project was to utilize the statistical technique of causal mediation analysis (CMA) to determine whether muscle wasting is a confounding mediator of muscle fatigue in response to breast tumor growth. We tested the hypothesis that breast tumor growth in experimental mice has a direct effect on muscle fatigue that is not mediated by muscle wasting.

Methods: Mammary tumors were induced in immunocompetent female C57BL/6 mice using a syngeneic tumor cell line (E0771) and in immunocompromised female NSG mice using breast cancer patient-derived orthotopic xenografts (BC-PDOXs). Muscle fatigue of the extensor digitorum longus (EDL) muscle was tested ex vivo. Four different models of causal mediation analysis (CMA) were used to calculate the average direct effects (ADE) of tumor growth on muscle fatigue and the average causal mediator effects (ACME) of muscle mass on muscle fatigue in our breast cancer models.

Results: Significant ADEs of tumor growth on muscle fatigue were observed in BCPDOX mice and E0771 tumor-bearing mice. None of the CMA models tested showed significant ACMEs of muscle mass on fatigue in response to breast tumor growth.

Conclusions: Our data support the hypothesis that muscle fatigue in mouse models of breast cancer occurs as a direct effect of tumor growth and that muscle wasting is likely not a mediator of this response. Considering the data in our study, we propose that the criteria used to diagnose cachexia in patients with breast cancer be reexamined to differentiate between skeletal muscle fatigue and skeletal muscle wasting. 


\section{INTRODUCTION}

Cancer is associated with peripheral toxicity, in which tissues and organ systems adjacent to and distant from the primary tumor can be affected by tumor cell proliferation, tumor growth, and/or the body's physiological responses to this stressor [1, 2]. Skeletal muscle is one of the organ systems that can respond to tumor growth, with different tumor types inducing differing responses in skeletal muscle, including varying degrees of muscle wasting and muscle dysfunction [3]. The term cancer cachexia has been used to describe these tumor-induced alterations in skeletal muscle over many millennia [4]. Despite these long standing observations, cancer cachexia currently lacks a universally accepted set of clinical parameters for diagnosis in patients. Importantly, this lack of consensus on what constitutes cachexia has hindered the development of therapeutic strategies to treat the condition. Cachexia remains a clinically relevant medical issue, is estimated to account for up to $20 \%$ of cancer mortality and is associated with reduced tolerance to tumor-directed therapies [5].

A defining characteristic of cancer cachexia is the loss of body weight, comprised primarily of skeletal muscle and adipose tissue, associated with tumor growth $[3,5]$. Muscle weakness is a symptom of muscle mass loss, as smaller muscles produce less force (i.e. decreased maximal strength). This has led to the dogma that cancer-induced muscle dysfunction is linked to muscle wasting. However, the muscle weakness or decreased maximal strength that results from muscle wasting is different from muscle fatigue, which can be defined as a decrease in muscle force due to repeated contractions or extended periods of exertion, and can occur in the absence of muscle wasting [6, 7]. Recently, Vanhoutte et al. [8] analyzed differences in overall survival 
(OS) when patients were diagnosed with cachexia using two separate sets of criteria.

In one set of criteria, there is a reliance on the percentage of body weight loss, changes in body mass index (BMI) and changes in appendicular skeletal muscle index [9]. In the second set of criteria, factors such as inflammation, muscle fatigue, and decreased muscle strength were assessed in addition to the percentage of body weight loss [10]. The study supported decreased survival for patients diagnosed with cachexia, regardless of the set of criteria utilized. However, the study also demonstrated that weight loss alone had little significant impact on survival curves, while also showing a prognostic benefit for more inclusive criteria for diagnosing cachexia [8]. While the goal of these studies was to provide general criteria that could be applied to patients with varying cancer diagnoses, patient responses often vary significantly among different tumor types. Therefore, an understanding of the clinical implications of different cancer types on skeletal muscle mass and function is required in order to develop effective therapies specific to the cancer type.

The working hypothesis that our laboratory has been actively working on (REFs) is that breast cancer is associated with greater muscle fatigue, a response that is independent of muscle wasting. A significant percentage of patients with breast cancer report fatigue [11-13], and our data in two different mouse models of breast tumor growth support this hypothesis $[14,15]$. Specifically, growth of breast cancer patientderived orthotopic xenografts (BC-PDOXs) in immunocompromised mice induced a greater rate of muscle fatigue that occurred in the absence of muscle wasting [15]. Additionally, growth of murine syngeneic breast tumors in C57BL/6 mice was associated with a greater rate of muscle fatigue that occurred with varying degrees of 
muscle wasting [14]. Therefore, the mechanisms promoting muscle fatigue during tumor growth are likely different from the mechanisms contributing to muscle wasting during tumor growth.

The purpose of this project was to utilize the statistical technique of causal mediation analysis (CMA) to determine if muscle wasting is a mediator of the muscle fatigue response to breast tumor growth. CMA is a statistical technique that allows for the unbiased quantification of whether a confounding mediator contributes to the observed responses to an experimental intervention [16-18]. We tested the hypothesis that breast tumor growth has a direct effect on muscle fatigue that is not mediated by muscle wasting in experimental mice. Specifically, we wanted to calculate the average direct effects (ADE) of breast tumor growth on muscle fatigue and the average causal mediator effects (ACME) of muscle mass on muscle fatigue in two breast tumor models. Analyses were completed in these different murine models to specifically compare shorter periods of tumor growth to longer durations (i.e. 4 weeks vs. 24 weeks) as well as the species of tumor grown in the models (i.e. mouse tumor vs. human tumor). The data support our hypothesis, that muscle fatigue in murine breast cancer occurs as a direct effect of tumor growth and that muscle mass is likely not a mediator of this response. Considering the data in our study, we propose that the criteria used to diagnose cachexia in patients with breast cancer be reexamined to differentiate between skeletal muscle fatigue and skeletal muscle wasting, and consider how each uniquely influences patients' quality of life and overall survival. 


\section{METHODS}

Previously published data. For this study, we utilized existing muscle fatigue and muscle mass data in two different models of breast tumor growth in experimental mice. Complete data for skeletal muscle responses to E0771 tumor growth in mice can be found in Bohlen et. al. [14] and Wilson et. al. [15], and complete data for skeletal muscle responses to BC-PDOX tumor growth in mice can be found in Wilson et. al. [15]. In our original publications using the E0771 tumor cell line, tumor-bearing mice were combined into a single tumor group. In this current study, we performed CMA with the mice combined into one tumor group as well as separated by the presence of tumor ulceration. Although we do not know the reason for the differing tumor phenotypes in response to E0771 tumor cell implantation, we observed differences in body mass at the time of euthanasia, providing rationale for analyzing these groups separately. The muscle fatigue and muscle mass data in mice with non-ulcerated and ulcerated E0771 tumors have not been previously published.

\section{Mouse models of mammary tumor growth.}

E0771 syngeneic mammary tumor growth. Mammary tumors were induced in female mice using a syngeneic tumor cell line (E0771) in C57BL/6 mice, as previously described $[14,15]$. Female C57BL/6 mice (stock \#: 000664) were purchased from The Jackson Laboratory $(n=30)$ and randomly assigned to three groups: control mice (Con; $n=8)$, mice that were euthanized following 2 weeks of tumor growth $(2 W K, n=5)$, and mice that were euthanized following 4 weeks of tumor growth $(4 \mathrm{WK}, \mathrm{n}=17)$. Preliminary studies were completed, in which it was determined that the duration from tumor cell implantation to maximal tumor burden was 4 weeks (data not shown). After 4 weeks of 
tumor growth, mammary tumors presented as a heterogeneous mix of ulcerated and non-ulcerated tumors. Therefore, the $4 \mathrm{WK}$ group was further divided into mice that developed an ulcerated tumor $(n=7)$ and mice that developed a non-ulcerated tumor $(n=10)$ for final analyses.

BC-PDOX mammary tumor growth. BC-PDOX mice were produced by implanting freshly collected breast tumor tissue into the cleared mammary fat pad of NOD.CG-

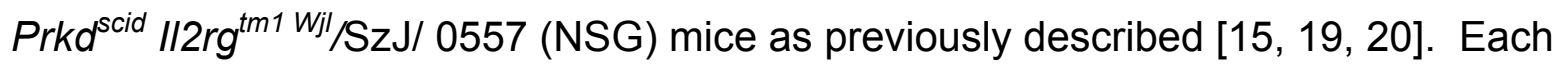
BC-PDOX model was authenticated with the original patient's biopsy using genomic DNA and short tandem repeat based PCR amplification (Arizona University Genomics Core Facility, Tucson, AZ, USA). Routine human and mouse pathogen screening was performed on original and passaged tissue by Charles River Laboratories (Wilmington, MA, USA). RNA sequence and whole exome analysis of the original biopsy and passage 1 BC-PDOX further confirmed relatedness of samples and stability of genomic alterations identified in BC patient and the corresponding PDOX. Expression of clinically relevant $\mathrm{BC}$ receptors correlated with the original $\mathrm{BC}$ biopsy. BC-PDOX mice $(n=8 ;$ age $=23 \pm 6$ weeks $)$ were compared to non-tumor NSG mice $(n=6$; age $=11 \pm 1$ weeks) as well as NSG mice that received human BC-PDOXs which did not engraft (Surgical-Con; $\mathrm{n}=5$; age $=51 \pm 16$ weeks). Clinical information on specific BC-PDOXs utilized, including tumor subtype and tumor stage classification, were previously published [15]. Mice were housed in the animal vivarium at West Virginia University in an ABSL-2 level room following tumor cell or BC-PDOX implantation, at $22^{\circ} \mathrm{C}$ under a 12:12-h light-dark cycle and received food and water ab libitum. Tumor growth was assessed by veterinary staff based on the approved WVU Tumor Development and 
Monitoring Policy. The average duration from tumor implantation to maximal tumor burden was $133 \pm 71$ days (range: 39-265 days). All animal experiments were approved by the Institutional Animal Care and Use Committee at West Virginia University.

Ex vivo muscle physiological analysis. Muscle contractile properties were examined in the extensor digitorum longus (EDL) muscles of experimental mice using methods as previously described $[14,15,21-24]$. Briefly, muscles were transferred to an oxygenated tissue bath containing Ringer's solution $(100 \mathrm{mM} \mathrm{NaCl}, 4.7 \mathrm{mM} \mathrm{KCl}, 3.4$ $\mathrm{mM} \mathrm{CaCl}_{2}, 1.2 \mathrm{mM} \mathrm{KH}_{2} \mathrm{PO}_{4}, 1.2 \mathrm{mM} \mathrm{MgSO}$, $25 \mathrm{mM} \mathrm{HEPES}$, and $5.5 \mathrm{mM}$ D-glucose) maintained at $22^{\circ} \mathrm{C}$. Muscle length was adjusted to optimal length (Lo), which produced the maximal twitch response. With the muscle set at Lo, muscle fatigue was analyzed using a repeated stimulation protocol lasting 6 min and consisting of repeated $40 \mathrm{~Hz}$ tetanic trains that occurred once every second and lasted $330 \mathrm{~ms}$ [25]. LabChart software V7 (ADInstruments, RRID: SCR_001620) was used to obtain the area under the fatigue curve $(A \cup C)$, which was used as a measure of total force produced during the fatigue protocol. The AUC value and corresponding EDL muscle mass for each muscle were used in CMA calculations.

\section{Statistical Analyses.}

Causal Mediation Analysis. The primary method of analysis in the present study was CMA, using a model-based inference strategy as previously described [26]. Briefly, a model-based CMA requires the fitting of two separate linear regression (LR) models to the data: a "mediator model," where the mediator variable (muscle mass) is regressed on the intervention variable (tumor), and an "outcome model," where the outcome 
variable (muscle fatigue, presented as $A U C$ ) is regressed on the intervention variable while controlling for the mediator (Figure 1A). Additional covariates can be added to the LR models to control for their effect on the mediator and/or the outcome variable. These LR models were then passed to the mediate function of the "mediation" Rpackage for calculation of $A C M E$ and $A D E$ and their associated uncertainty estimates over a number of simulations. In this analysis, we utilized 10,000 simulations per CMA for calculation of quasi-Bayesian $95 \%$ confidence intervals. Standard multiple regression was conducted in R v3.5.0 [27]. Two R packages, "DescTools" v0.99.28 [28] and "olsrr" v0.5.2 [29], were utilized for appropriate assumption checking after fitting linear models. Assumptions tested include the normality of dependent variables, absence of univariate outliers, non-multicollinearity, homoscedasticity, and absence of regression outliers. No assumptions were found to be violated. Models tested are summarized as follows:

PDOX Model Summaries (Figure 1B)

CMA 1 (Mediator LR): EDL mass subgroup (naive, surgical control, tumor-bearing) CMA 1 (Outcome LR): AUC EDL mass + subgroup

CMA 2 (Mediator LR): EDL mass group (control, tumor-bearing) + age in weeks CMA 2 (Outcome LR): AUC EDL mass + group + age in weeks E0771 Model Summaries (Figure 1C)

CMA 3 (Mediator LR): EDL mass tumor type (no tumor, ulcerated, non-ulcerated) CMA 3 (Outcome LR): AUC EDL mass + tumor type CMA 4 (Mediator LR): EDL mass group (control, tumor-bearing) + tumor ulceration status 
CMA 4 (Outcome LR): AUC EDL mass + group + tumor ulceration status

Descriptive and comparative statistics. Data are presented as means \pm SD. For E0771 experiments, tumor volume, body mass, AUC and muscle mass were analyzed using a one-way analysis of variance (ANOVA) for 4 experimental groups (Con, 2WK, 4WK non-ulcerated, 4WK-ulcerated). A two-way ANOVA with Tukey's post-hoc test was used to analyze fatigue curves, comparing force output over time during the 6-minute fatigue protocol. Statistical inferences were based on two-sided tests and the null hypothesis was rejected when $p<0.05$. 


\section{RESULTS}

Causal mediation analysis in the BC-PDOX model. In our original publication, skeletal muscles from BC-PDOX mice fatigued at a greater rate than both naïve control mice and surgical control mice, and there were no significant differences in muscle mass of the tested skeletal muscle [15]. Data presented in Figure 2 represent the mean and $95 \%$ confidence intervals for the ADE and ACME tested. In the four different CMA models we tested, there were significant values for the direct effect (ADE) of tumor growth on the muscle fatigue response, with a negative effect size in all cases, indicating that tumor growth results in a decreased AUC value that reflects increased fatigability. However, muscle mass was not a significant mediator (ACME) of the muscle fatigue response (Table 1). The ADE was strongest when comparing the BC-PDOX mice and the naïve control mice (Figure 2A), while the comparison of BC-PDOX mice to surgical controls reached a p-value equal to 0.05 (Figure 2B). When both control groups were combined into a single group, the ADE was highly significant (Figure $2 \mathrm{C}$ ). When the age of the experimental animals at euthanasia was used as a covariate in the CMA model, the ADE was also highly significant (Figure 2D). No model tested showed a significant ACME of muscle mass. Collectively, these data demonstrate that tumor growth in the BC-PDOX mouse model directly contributes to the muscle fatigue response and that muscle mass is likely not a mediator of muscle fatigue in this mouse model of breast cancer.

Skeletal muscle responses to E0771 mammary tumor growth. One million E0771 tumor cells were implanted into the mammary fat pads of female wild type C57BL/6 
mice, as previously described [14]. Following 4 weeks of tumor growth, both nonulcerated tumors and ulcerated tumors were observed in experimental mice (Figure 3A). The volume of these tumors at the time of euthanasia differed significantly, such that $2 \mathrm{WK}$ tumors averaged $397.3 \mathrm{~mm}^{3} \pm 33.8,4 \mathrm{WK}$ non-ulcerated tumors averaged $3927 \mathrm{~mm}^{3} \pm 571.3$, and 4 WK ulcerated tumors averaged $1830 \mathrm{~mm}^{3} \pm 405.8$ (Figure 3B). Upon removal of the tumor, the body weight of Con, $2 \mathrm{WK}$ and $4 \mathrm{WK}$ non-ulcerated mice were not significantly different. However, 4WK ulcerated mice weighed $19.6 \%$, $17.4 \%$, and $11.6 \%$ less than Con, $2 \mathrm{WK}$, and $4 \mathrm{WK}$ non-ulcerated mice at the time of euthanasia, respectively (Figure 3C). When body weight was tracked from the time of tumor cell implantation to euthanasia, $2 \mathrm{WK}$ mice gained 3.0\%, $4 \mathrm{WK}$ non-ulcerated mice lost $8.6 \%$ and 4 WK ulcerated mice lost $17.4 \%$ body weight (Figure 3D). Therefore, we were able to induce cachexia (i.e. loss of body weight) in wild type mice in response to 4 weeks of E0771 tumor growth and ulcerated tumors had a more dramatic effect on overall body weight loss than non-ulcerated tumors, despite a smaller tumor volume.

Muscle fatigue was analyzed using a repeated stimulation protocol, with the fatigue index calculated as the percent difference of each contraction from the initial contraction of the protocol. When the fatigue index curve was plotted, a clear pattern emerged with respect to the duration of tumor exposure. There were no differences in the fatigue curves of EDL muscles from Con and 2WK mice. However, the fatigue curves of EDL muscles from both 4WK non-ulcerated and 4WK ulcerated mice were shifted down and to the left compared to Con and $2 \mathrm{WK}$, demonstrating a faster rate of force loss with repeated contractions. This shift occurred during the first 80 seconds of the fatigue protocol (Figure 3E). Additionally, there was a significantly lower AUC when 
comparing the fatigue curves of EDL muscles from mice exposed to 4 weeks of tumor growth compared to the AUC from Con mice (Figure 3F). Mass of the EDL muscle was significantly lower following 4 weeks of tumor growth, compared to control mice and 2WK mice. The loss of EDL muscle mass with tumor growth was not different between 4WK non-ulcerated and 4WK ulcerated mice (Figure 3G).

Causal mediation analysis in the E0771 model. Data presented in Figure 4 represent the mean and $95 \%$ confidence intervals for the ADE and ACME tested. In CMA models tested, there were significant values for the ADE of tumor growth on the muscle fatigue response. However, muscle mass was not a significant mediator of the muscle fatigue response in any model tested (Table 1). The ADE was strongest when comparing the $4 \mathrm{WK}$ non-ulcerated mice and control mice (Figure 4A), while the comparison of 4WK ulcerated mice to control mice reached a p-value of 0.05 (Figure 4B). When both tumor groups were combined into a single group, the ADE was highly significant (Figure 4C). When the tumor ulceration phenotype was used as a covariate in the CMA model, the ADE was also highly significant (Figure 4D). Thus, tumor growth in the syngeneic E0771 tumor xenograft model directly contributes to muscle fatigue and muscle mass is likely not a mediator of muscle fatigue in this mouse model of breast cancer. 


\section{DISCUSSION}

The purpose of this study was to utilize the statistical technique of CMA to determine if muscle wasting is a mediator of the muscle fatigue response to breast tumor growth. We found that breast tumor growth in mice contributes to muscle fatigue directly, and this response is likely not mediated by muscle wasting. These conclusions are based on data acquired in two separate mouse models of breast cancer: 1) the BCPDOX model in which muscle mass was not significantly different between tumorbearing and control mice after an average of 24 weeks of tumor growth; and 2) the E0771 syngeneic xenograft model in which muscle mass was statistically different between tumor-bearing mice and control mice after 4 weeks of tumor growth. Multiple CMA models were analyzed in each breast cancer model to test for mediator effects of muscle wasting as well as covariates within each breast cancer model. The strength of CMA is the ability to obtain "unbiased estimates of the part of the effect of an intervention that is mediated through a specific variable" [16]. In the current study, the "effect" was muscle fatigue, the "intervention" was breast tumor growth, and the "mediator variable" was muscle mass.

In the PDOX model, we anticipated that muscle mass would not be a significant mediator of muscle fatigability because muscle mass was not significantly affected in tumor-bearing animals. Unsurprisingly, muscle mass was not found to be a significant mediator of muscle fatigability in this model $(p=0.41)$. Even if this result were statistically significant, the effect size of -152 is less than $10 \%$ of tumor's direct effect on muscle fatigability of $-1660(p<0.0001)$, indicating that even if muscle mass were a 
mediating factor, the proportion of the effect attributable to change in muscle mass is very small.

In the EO771 model, many of the mice did exhibit significant muscle atrophy and body weight loss, particularly in mice with ulcerated tumors. It would therefore be more likely for this model to show a relationship between muscle mass and muscle fatigability. However, muscle mass was not found to be a significant mediator of muscle fatigability $(p=0.61)$. Even if this result were statistically significant, the effect size of +168 is in the opposite direction of, and much smaller than, tumor's effect on fatigability, $-2990(p<0.0001)$. This means that if muscle mass were a mediating factor in this model, muscle atrophy would be protecting against fatigue, not contributing to it. An alternative interpretation of these data could be that muscle fatigue is protecting against muscle atrophy.

The strength of CMA is the ability to obtain "unbiased estimates of the part of the effect of an intervention that is mediated through a specific variable" [16]. In the current study, the "effect" was muscle fatigue, the "intervention" was breast tumor growth, and the "mediator variable" was muscle mass. Since the CMA was not statistically significant for the mediator variable in the two breast tumor models utilized, but was significant for a direct effect of tumor growth on fatigue, we concluded that muscle wasting was not a mediator of the muscle fatigue observed in response to breast tumor growth. This observation is strengthened by the use of two different mouse models of breast cancer with varying durations of tumor growth. However, our enthusiasm for this conclusion is slightly tempered by the fact that it is based on the specific methodology our laboratory utilizes to assess muscle fatigue (i.e., ex vivo muscle stimulation) [14, 15, 
21-24, 30]. Further analyses using additional models of fatigue in experimental animals and humans are warranted, to verify and validate these conclusions.

Evidence for negative effects on skeletal muscle mitochondria have been reported in experimentally-induced cancer, and this is often associated with body weight loss and muscle wasting. A recent study demonstrated mitochondrial dysfunction in muscles from mice with syngeneic colon adenocarcinoma xenograft tumors, in addition to a $20 \%$ loss of body weight and lower skeletal muscle masses [31]. Supporting this effect in breast cancer, Gilliam et al [32] show a reduced mitochondrial respiration rate following breast tumor growth and chemotherapy in mice. Interestingly, recent studies suggest the possibility that mitochondrial dysfunction occurs prior to the onset of muscle wasting $[33,34]$. This suggests a temporal response to tumor growth within skeletal muscle may exist, in which mitochondrial function may be susceptible to the early stages of tumor growth, followed by muscle wasting in the later stages of disease. In the E0771 breast tumor model, we show that 4 weeks of tumor growth is associated with lower body mass and skeletal muscle mass in addition to a greater rate of muscle fatigue [14].However, data in the BC-PDOX model system are counter to this hypothesis, since we observed greater muscle fatigue in the absence of muscle wasting in mice following 24 weeks of tumor growth and with positive metastases [15]. Therefore, while mitochondrial dysfunction may be a common mechanism between a number of cancer models [31], this dysfunction is not necessarily mechanistically linked to the onset or occurrence of muscle wasting.

In a recent review on the topic of cancer cachexia, patients diagnosed with breast cancer were identified as having the second lowest prevalence of cachexia and 
the lowest average weight loss among patients diagnosed with eight different cancer types [3]. Despite this relatively low percentage of body weight loss, a significant percentage of patients with breast cancer report fatigue, which can occur in response to both tumor growth itself and cancer-associated therapies [35-37]. In an attempt to determine the molecular alterations associated with the skeletal muscle responses in patients with breast cancer, we analyzed the global transcriptional profile of skeletal muscle from early stage non-metastatic breast cancer patients as well as from BCPDOX mice $[14,15]$. A subset of concordantly dysregulated genes in muscle from humans and mice exposed to human breast tumors was identified, and these genes predicted changes in metabolism as well as mitochondrial function. Multiple bioinformatics platforms predicted dysregulation of pathways involved in peroxisome proliferator activated receptor (PPAR) signaling, glucose/energy metabolism, regulation of lipid metabolism, and ATP generation via the electron transport chain. These transcriptional changes were further supported by the skeletal muscle functional data in BC-PDOX mice showing a greater rate of muscle fatigue following breast tumor growth. A greater rate of fatigue occurred despite no significant differences in the muscle mass of the EDL muscle following tumor growth in this model [15]. Therefore, the BC-PDOX model recapitulates the clinical observation of breast cancer-induced muscle fatigue in the absence of muscle wasting. Clinicians should be aware that patients with breast cancer may not have dramatic changes in body weight and/or muscle mass during tumor growth or tumor-directed therapies. However, this does not necessarily imply that muscle dysfunction, in the form of persistent fatigue, is not adversely affecting the quality of life of the patient. Based on our transcriptional data in human patients, 
skeletal muscle is responding at the early stages of breast tumor growth, and these transcriptional changes would predict the dysregulation of pathways associated with muscle fatigue [15].

Previous data from our laboratory supports an association of greater IL-15 with increased mitochondrial density in skeletal muscle [23, 38-40]. Furthermore, in vitro data from our laboratory [41] and others [42] supports the direct effect of IL-15 binding in the stimulation of pathways upstream of mitochondrial biogenesis within skeletal muscle. We utilized this mechanism of action in a breast cancer model by inducing mammary tumors in transgenic mice overexpressing IL-15 in skeletal muscle, which was then actively secreted into the circulation [43]. In our model system, E0771 mammary tumor growth in IL15TG mice was associated with a rescue of muscle fatigue following 4 weeks of tumor growth. However, there was approximately $23 \%$ loss of muscle mass in IL15TG mice exposed to tumor growth, compared to IL15TG mice not exposed to tumor growth. Therefore, in this model system, we were able to attenuate the expected increase in muscle fatigability induced by E0771 mammary tumor growth, despite a significant loss of muscle mass. Therefore, these data suggest that although experimentally-induced breast cancer is associated with muscle atrophy in this model, the mechanisms that mediate muscle wasting and muscle fatigue are independent of one another since we could rescue the greater amount of fatigue in atrophied muscles.

A limitation of our study, which we identified above, is the utilization of only one method to test muscle fatigue. In our ex vivo preparation, the EDL muscle is electrically stimulated in a tissue chamber that contains Ringers solution that is temperature controlled and oxygenated. This model represents the inherent contractile and fatigue 
properties of the muscle, since we can eliminate any effects of altered blood flow or nervous system input to the muscle during fatiguing contractions. Despite these caveats, the conclusions of this current study should be confirmed in additional animal models of fatigue (i.e., in situ muscle stimulation; whole animal exercise) to verify and validate our conclusions. An additional limitation of this study is the exclusive use of female mice. While the use of female mice for the study of breast cancer is justified by the $100 x$ greater incidence of this disease in women compared to men [44], this limits the conclusions we can make on the overall role of sex in muscle fatigue. Skeletal muscle tissue is one of the top three most sexually dimorphic tissues in humans, along with adipose tissue and mammary tissue [45]. For example, men lose more weight in response to tumor growth, lose more skeletal muscle mass, and have approximately double the incidence of cachexia as women with cancer [46, 47]. Furthermore, in men, the loss of muscle function correlates with loss of muscle mass, but this is not the case in women. In other words, women experience muscle dysfunction without a commensurate loss of muscle mass [48]. This observation can be attributed to multiple potential mechanisms, including a recently described phenomenon whereby women with cancer exhibit a significant infiltration of non-contractile tissue into skeletal muscle, significant metabolic differences between males and females, and/or differences in hormonal concentrations in males and females [7]. Future studies should include male mice to address the effects of breast cancer in males as well as the effect of sex on muscle fatigue and muscle wasting in response to breast tumor growth.

In conclusion, our CMA analysis provides evidence that muscle fatigue, in experimentally-induced breast cancer, occurs as a direct response to breast tumor 
growth and is likely not mediated by changes in muscle mass. This finding is consistent with observations in patients with breast cancer, since fatigue is a commonly reported symptom while body weight loss is comparatively low in this population [3]. Therefore, the criteria used to define and diagnose cachexia in breast cancer patients should be reexamined to differentiate muscle fatigue and muscle wasting. The recognition that the underlying mechanisms contributing to muscle fatigue and muscle wasting are different, and that muscle fatigue may occur in these patients without concomitant body weight loss, is a first step in the ability to design and implement therapies to improve the quality of life of patients with breast cancer. 
COMPLIANCE WITH ETHICAL STANDARDS

Funding: This research was funded by grants from the National Institute Of General Medical Sciences of the National Institutes of Health (P20GM121322; P. Lockman), American Cancer Society Institutional Research Grant (09-061-04; E. Pistilli) and the WVCTSI through the National Institute of General Medical Sciences (U54GM104942; S. Hodder). Additional support for this research was provided by WV-INBRE (P20GM103434), CA148671 (E.N. Pugacheva), from the NIH/NCl in part by a NIH/NCRR5 P20-RR016440-09. The WVU HSC Core Facilities were supported by the NIH grants P30- RR032138/GM103488, S10-RR026378, S10-RR020866, S10OD016165, and P20GM103434. The content is solely the responsibility of the authors and does not necessarily represent the official views of the National Institutes of Health.

Conflict of Interest: Emidio E. Pistilli declares he has no conflicts of interest. Hannah E. Wilson declares she has no conflicts of interest. David A. Stanton declares he has no conflicts of interest. Joseph Bohlen declares he has no conflicts of interest. Elena N. Pugacheva declares she has no conflicts of interest. Sijin Wen declares he has no conflicts of interest. Sarah L. McLaughlin declares she has no conflicts of interest.

Ethical Approval: All applicable international, national and institutional guidelines for the care and use of animals were followed. This article does not contain any studies with human participants performed by any of the authors. 


\section{REFERENCES}

1. DeBerardinis, R. J. \& Chandel, N. S. (2016) Fundamentals of cancer metabolism, Sci Adv. 2, e1600200.

2. Hanahan, D. \& Weinberg, R. A. (2000) The hallmarks of cancer, Cell. 100, 5770.

3. Baracos, V. E., Martin, L., Korc, M., Guttridge, D. C. \& Fearon, K. C. H. (2018) Cancer-associated cachexia, Nat Rev Dis Primers. 4, 17105.

4. Katz, A. M. \& Katz, P. B. (1962) Diseases of the heart in the works of Hippocrates, Br Heart J. 24, 257-64.

5. Argiles, J. M., Busquets, S., Stemmler, B. \& Lopez-Soriano, F. J. (2014) Cancer cachexia: understanding the molecular basis, Nat Rev Cancer. 14, 754-62.

6. Evans, W. J. \& Lambert, C. P. (2007) Physiological basis of fatigue, Am J Phys Med Rehabil. 86, S29-46.

7. Neefjes, E. C. W., van den Hurk, R. M., Blauwhoff-Buskermolen, S., van der Vorst, M., Becker-Commissaris, A., de van der Schueren, M. A. E., Buffart, L. M. \& Verheul, H. M. W. (2017) Muscle mass as a target to reduce fatigue in patients with advanced cancer, J Cachexia Sarcopenia Muscle. 8, 623-629.

8. Vanhoutte, G., van de Wiel, M., Wouters, K., Sels, M., Bartolomeeussen, L., De Keersmaecker, S., Verschueren, C., De Vroey, V., De Wilde, A., Smits, E., Cheung, K. J., De Clerck, L., Aerts, P., Baert, D., Vandoninck, C., Kindt, S., Schelfhaut, S., Vankerkhoven, M., Troch, A., Ceulemans, L., Vandenbergh, H., Leys, S., Rondou, T., Dewitte, E., Maes, K., Pauwels, P., De Winter, B., 
Van Gaal, L., Ysebaert, D. \& Peeters, M. (2016) Cachexia in cancer: what is in the definition?, BMJ Open Gastroenterol. 3, e000097.

9. Fearon, K., Strasser, F., Anker, S. D., Bosaeus, I., Bruera, E., Fainsinger, R. L., Jatoi, A., Loprinzi, C., MacDonald, N., Mantovani, G., Davis, M., Muscaritoli, M., Ottery, F., Radbruch, L., Ravasco, P., Walsh, D., Wilcock, A., Kaasa, S. \& Baracos, V. E. (2011) Definition and classification of cancer cachexia: an international consensus, Lancet Oncol. 12, 489-95.

10. Evans, W. J., Morley, J. E., Argiles, J., Bales, C., Baracos, V., Guttridge, D., Jatoi, A., Kalantar-Zadeh, K., Lochs, H., Mantovani, G., Marks, D., Mitch, W. E., Muscaritoli, M., Najand, A., Ponikowski, P., Rossi Fanelli, F., Schambelan, M., Schols, A., Schuster, M., Thomas, D., Wolfe, R. \& Anker, S. D. (2008) Cachexia: a new definition, Clin Nutr. 27, 793-9.

11. Bower, J. E., Ganz, P. A., Desmond, K. A., Rowland, J. H., Meyerowitz, B. E. \& Belin, T. R. (2000) Fatigue in breast cancer survivors: occurrence, correlates, and impact on quality of life, J Clin Oncol. 18, 743-53.

12. Cella, D., Lai, J. S., Chang, C. H., Peterman, A. \& Slavin, M. (2002) Fatigue in cancer patients compared with fatigue in the general United States population, Cancer. 94, 528-38.

13. Curt, G. A., Breitbart, W., Cella, D., Groopman, J. E., Horning, S. J., Itri, L. M., Johnson, D. H., Miaskowski, C., Scherr, S. L., Portenoy, R. K. \& Vogelzang, N. J. (2000) Impact of cancer-related fatigue on the lives of patients: new findings from the Fatigue Coalition, Oncologist. 5, 353-60. 
14. Bohlen, J., McLaughlin, S. L., Hazard-Jenkins, H., Infante, A. M., Montgomery, C., Davis, M. \& Pistilli, E. E. (2018) Dysregulation of metabolicassociated pathways in muscle of breast cancer patients: preclinical evaluation of interleukin-15 targeting fatigue, J Cachexia Sarcopenia Muscle. 9, 701-714.

15. Wilson, H. E., Rhodes, K. K., Rodriguez, D., Chahal, I., Stanton, D. A., Bohlen, J., Davis, M., Infante, A. M., Hazard-Jenkins, H., Klinke, D. J., Pugacheva, E. N. \& Pistilli, E. E. (2019) Human Breast Cancer Xenograft Model Implicates Peroxisome Proliferator-activated Receptor Signaling as Driver of Cancer-induced Muscle Fatigue, Clin Cancer Res. 25, 2336-2347.

16. Nuzzo, J. L., Finn, H. T. \& Herbert, R. D. (2019) Causal Mediation Analysis Could Resolve Whether Training-Induced Increases in Muscle Strength are Mediated by Muscle Hypertrophy, Sports Med.

17. Pearl, J. (2014) Interpretation and identification of causal mediation, Psychol Methods. 19, 459-81.

18. Imai, K., Keele, L. \& Tingley, D. (2010) A general approach to causal mediation analysis, Psychol Methods. 15, 309-34.

19. Dunphy, K. A., Tao, L. \& Jerry, D. J. (2010) Mammary epithelial transplant procedure, J Vis Exp.

20. DeRose, Y. S., Gligorich, K. M., Wang, G., Georgelas, A., Bowman, P., Courdy, S. J., Welm, A. L. \& Welm, B. E. (2013) Patient-derived models of human breast cancer: protocols for in vitro and in vivo applications in tumor 
biology and translational medicine, Curr Protoc Pharmacol. Chapter 14, Unit14 23.

21. O'Connell, G., Guo, G., Stricker, J., Quinn, L. S., Ma, A. \& Pistilli, E. E. (2015) Muscle-specific deletion of exons 2 and 3 of the IL15RA gene in mice: effects on contractile properties of fast and slow muscles, J Appl Physiol (1985). 118, 437-48.

22. Pistilli, E. E., Alway, S. E., Hollander, J. M. \& Wimsatt, J. H. (2014) Aging alters contractile properties and fiber morphology in pigeon skeletal muscle, $J$ Comp Physiol B. 184, 1031-9.

23. Pistilli, E. E., Bogdanovich, S., Garton, F., Yang, N., Gulbin, J. P., Conner, J. D., Anderson, B. G., Quinn, L. S., North, K., Ahima, R. S. \& Khurana, T. S. (2011) Loss of IL-15 receptor alpha alters the endurance, fatigability, and metabolic characteristics of mouse fast skeletal muscles, J Clin Invest. 121, 3120-32.

24. Pistilli, E. E., Bogdanovich, S., Goncalves, M. D., Ahima, R. S., Lachey, J., Seehra, J. \& Khurana, T. (2011) Targeting the activin type IIB receptor to improve muscle mass and function in the mdx mouse model of Duchenne muscular dystrophy, Am J Pathol. 178, 1287-97.

25. Burke, R. E., Levine, D. N., Tsairis, P. \& Zajac, F. E., 3rd (1973) Physiological types and histochemical profiles in motor units of the cat gastrocnemius, J Physiol. 234, 723-48. 
26. Imai, K., Keele, L., Tingley, D. \& Yamamoto, T. (2010) Causal Mediation Analysis Using R in Advances in Social Science Research Using R (Vinod, H. D., ed) pp. 129-154, Springer, New York.

27. Team, R. C. (2018) R: A language and environment for statistical computing in Vienna.

28. Signorel, A. (2019) Package "DescTools" in Tools for Descriptive Statistics pp. $1-591$

29. Hebbali, A. (2018) Package 'olsrr" in Tools for Building OLS Regression Models pp. 1-74

30. Pistilli, E. E., Bogdanovich, S., Mosqueira, M., Lachey, J., Seehra, J. \& Khurana, T. S. (2010) Pretreatment with a soluble activin type IIB receptor/Fc fusion protein improves hypoxia-induced muscle dysfunction, Am J Physiol Regul Integr Comp Physiol. 298, R96-R103.

31. Neyroud, D., Nosacka, R. L., Judge, A. R. \& Hepple, R. T. (2019) Colon 26 adenocarcinoma (C26)-induced cancer cachexia impairs skeletal muscle mitochondrial function and content, J Muscle Res Cell Motil. 40, 59-65.

32. Gilliam, L. A., Lark, D. S., Reese, L. R., Torres, M. J., Ryan, T. E., Lin, C. T., Cathey, B. L. \& Neufer, P. D. (2016) Targeted overexpression of mitochondrial catalase protects against cancer chemotherapy-induced skeletal muscle dysfunction, Am J Physiol Endocrinol Metab. 311, E293-301.

33. Brown, J. L., Rosa-Caldwell, M. E., Lee, D. E., Blackwell, T. A., Brown, L. A., Perry, R. A., Haynie, W. S., Hardee, J. P., Carson, J. A., Wiggs, M. P., Washington, T. A. \& Greene, N. P. (2017) Mitochondrial degeneration 
precedes the development of muscle atrophy in progression of cancer cachexia in tumour-bearing mice, J Cachexia Sarcopenia Muscle. 8, 926-938.

34. White, J. P., Puppa, M. J., Sato, S., Gao, S., Price, R. L., Baynes, J. W., Kostek, M. C., Matesic, L. E. \& Carson, J. A. (2012) IL-6 regulation on skeletal muscle mitochondrial remodeling during cancer cachexia in the ApcMin/+ mouse, Skelet Muscle. 2, 14.

35. Irvine, D., Vincent, L., Graydon, J. E., Bubela, N. \& Thompson, L. (1994) The prevalence and correlates of fatigue in patients receiving treatment with chemotherapy and radiotherapy. A comparison with the fatigue experienced by healthy individuals, Cancer Nurs. 17, 367-78.

36. Irvine, D. M., Vincent, L., Graydon, J. E. \& Bubela, N. (1998) Fatigue in women with breast cancer receiving radiation therapy, Cancer Nurs. 21, 12735.

37. Hofman, M., Ryan, J. L., Figueroa-Moseley, C. D., Jean-Pierre, P. \& Morrow, G. R. (2007) Cancer-related fatigue: the scale of the problem, Oncologist. 12 Suppl 1, 4-10.

38. Pistilli, E. E. \& Quinn, L. S. (2013) From anabolic to oxidative: reconsidering the roles of IL-15 and IL-15Ralpha in skeletal muscle, Exerc Sport Sci Rev. 41, 100-6.

39. Nadeau, L., Patten, D. A., Caron, A., Garneau, L., Pinault-Masson, E., Foretz, M., Haddad, P., Anderson, B. G., Quinn, L. S., Jardine, K., McBurney, M. W., Pistilli, E. E., Harper, M. E. \& Aguer, C. (2019) IL-15 improves skeletal muscle oxidative metabolism and glucose uptake in association with 
increased respiratory chain supercomplex formation and AMPK pathway activation, Biochim Biophys Acta Gen Subj. 1863, 395-407.

40. Quinn, L. S., Anderson, B. G., Conner, J. D., Pistilli, E. E. \& Wolden-Hanson, T. (2011) Overexpression of interleukin-15 in mice promotes resistance to diet-induced obesity, increased insulin sensitivity, and markers of oxidative skeletal muscle metabolism, Int J Interferon Cytokine Mediat Res. 3, 29-42.

41. O'Connell, G. C. \& Pistilli, E. E. (2015) Interleukin-15 directly stimulates prooxidative gene expression in skeletal muscle in-vitro via a mechanism that requires interleukin-15 receptor alpha, Biochem Biophys Res Commun. 458, 614-619.

42. Thornton, S. M., Krolopp, J. E. \& Abbott, M. J. (2016) IL-15 Mediates Mitochondrial Activity through a PPARdelta-Dependent-PPARalphaIndependent Mechanism in Skeletal Muscle Cells, PPAR Res. 2016, 5465804.

43. Quinn, L. S., Anderson, B. G., Strait-Bodey, L., Stroud, A. M. \& Argiles, J. M. (2009) Oversecretion of interleukin-15 from skeletal muscle reduces adiposity, Am J Physiol Endocrinol Metab. 296, E191-202.

44. Ly, D., Forman, D., Ferlay, J., Brinton, L. A. \& Cook, M. B. (2013) An international comparison of male and female breast cancer incidence rates, Int J Cancer. 132, 1918-26.

45. Gershoni, M. \& Pietrokovski, S. (2017) The landscape of sex-differential transcriptome and its consequent selection in human adults, BMC Biol. 15, 7. 
46. Anderson, L. J., Liu, H. \& Garcia, J. M. (2017) Sex Differences in Muscle Wasting, Adv Exp Med Biol. 1043, 153-197.

47. Wallengren, O., Iresjo, B. M., Lundholm, K. \& Bosaeus, I. (2015) Loss of muscle mass in the end of life in patients with advanced cancer, Support Care Cancer. 23, 79-86.

48. Stephens, N. A., Gray, C., MacDonald, A. J., Tan, B. H., Gallagher, I. J., Skipworth, R. J., Ross, J. A., Fearon, K. C. \& Greig, C. A. (2012) Sexual dimorphism modulates the impact of cancer cachexia on lower limb muscle mass and function, Clin Nutr. 31, 499-505. 


\section{FIGURES AND FIGURE LEGENDS}

\section{Figure 1: Causal mediation analysis models of muscle wasting and muscle}

\section{fatigue in response to breast tumor growth.}

Figure 1

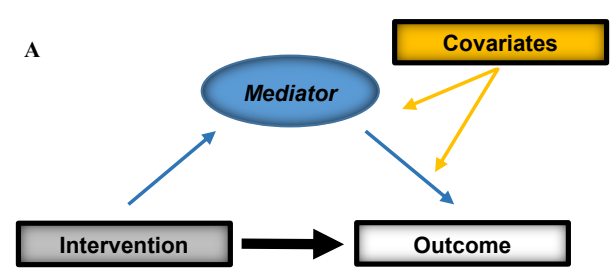

B
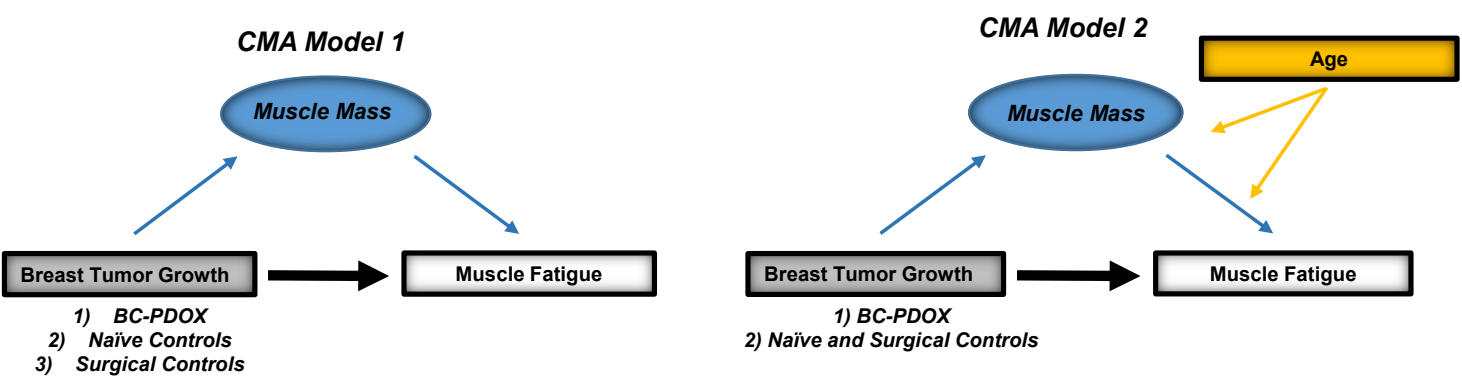

C

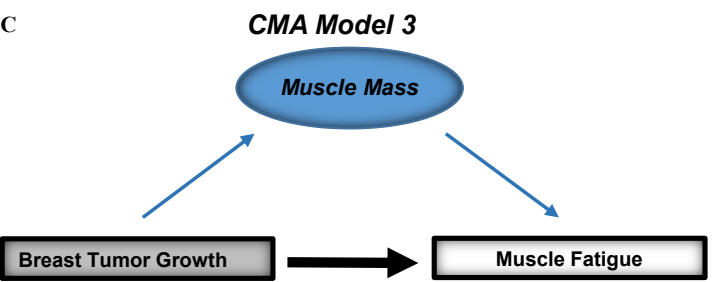

1) E0771 Ulcerated

2) EO771 Non-ulcerated

Muscle Fatigue

Breast Tumor Growth

1) $E 0771$

2) Naïve Controls

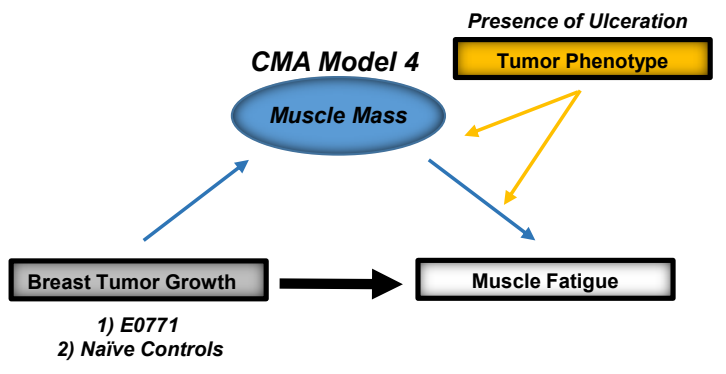

(A) Representative CMA diagram depicting the mediator linear regression and outcome linear regression models tested. (B) CMA Model 1 was generated to test the direct effects of BC-PDOX tumor growth on muscle fatigue (thick arrow), and the mediator effects of muscle mass the muscle fatigue response to tumor growth (blue, thin arrows). CMA Model 2 included the age of control mice as a covariate (yellow arrows). (B) CMA Model 3 was generated to test the direct effects of E0771 tumor growth on muscle fatigue (thick arrow), and the mediator effects of muscle mass the 
muscle fatigue response to tumor growth (blue, thin arrows). CMA Model 4 included the presence of a tumor ulceration as a covariate (yellow arrows).

\section{Figure 2: Causal mediation analysis in BC-PDOX mice.}

Figure 2

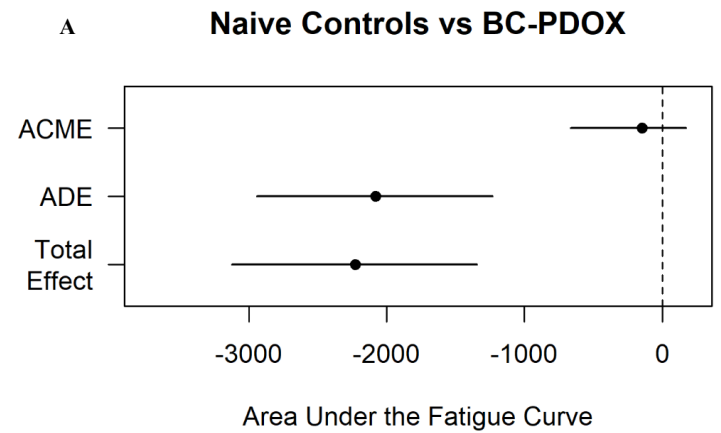

C All Controls vs BC-PDOX

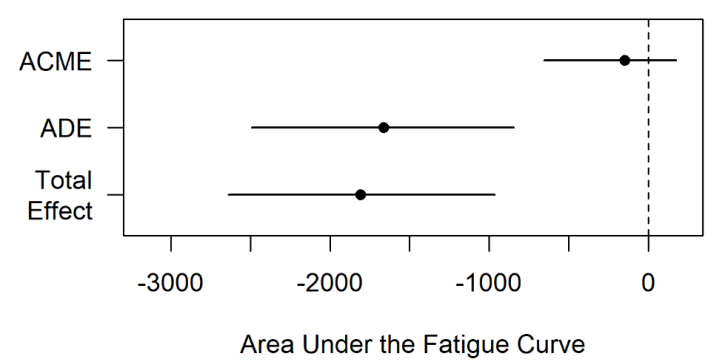

B Surgical Controls vs BC-PDOX

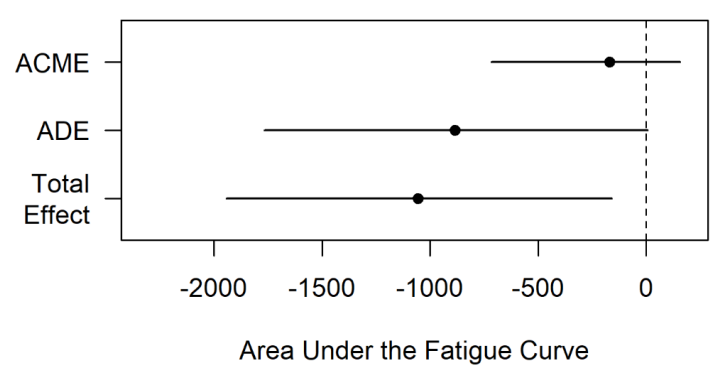

D All Controls vs BC-PDOX, Age Covariate

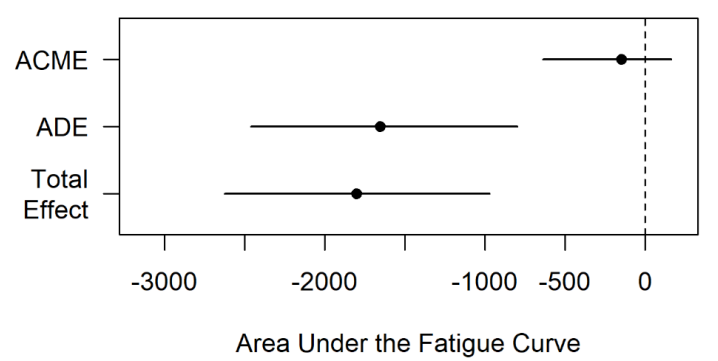

Graphical representation of the estimate and $95 \%$ confidence interval for the Total Effect, the Average Direct Effect (ADE) and the Average Causal Mediation Effect (ACME) for all CMA models tested. The direct effect of tumor growth on muscle fatigue was highly correlated with the total effect, while there was no significant mediator effect of muscle mass in comparing naïve control mice (A) or surgical control mice (B) to BCPDOX mice. Similar results were obtained when both control groups were combined 
into a single group for comparison to BC-PDOX (C), and when the age of the two control groups was used as a covariate in the analysis (D). Data presented as the mean and $95 \%$ confidence intervals for the ADE and ACME tested in these models.

Figure 3: E0771 tumor growth in C57BL/6 mice.

Figure 3
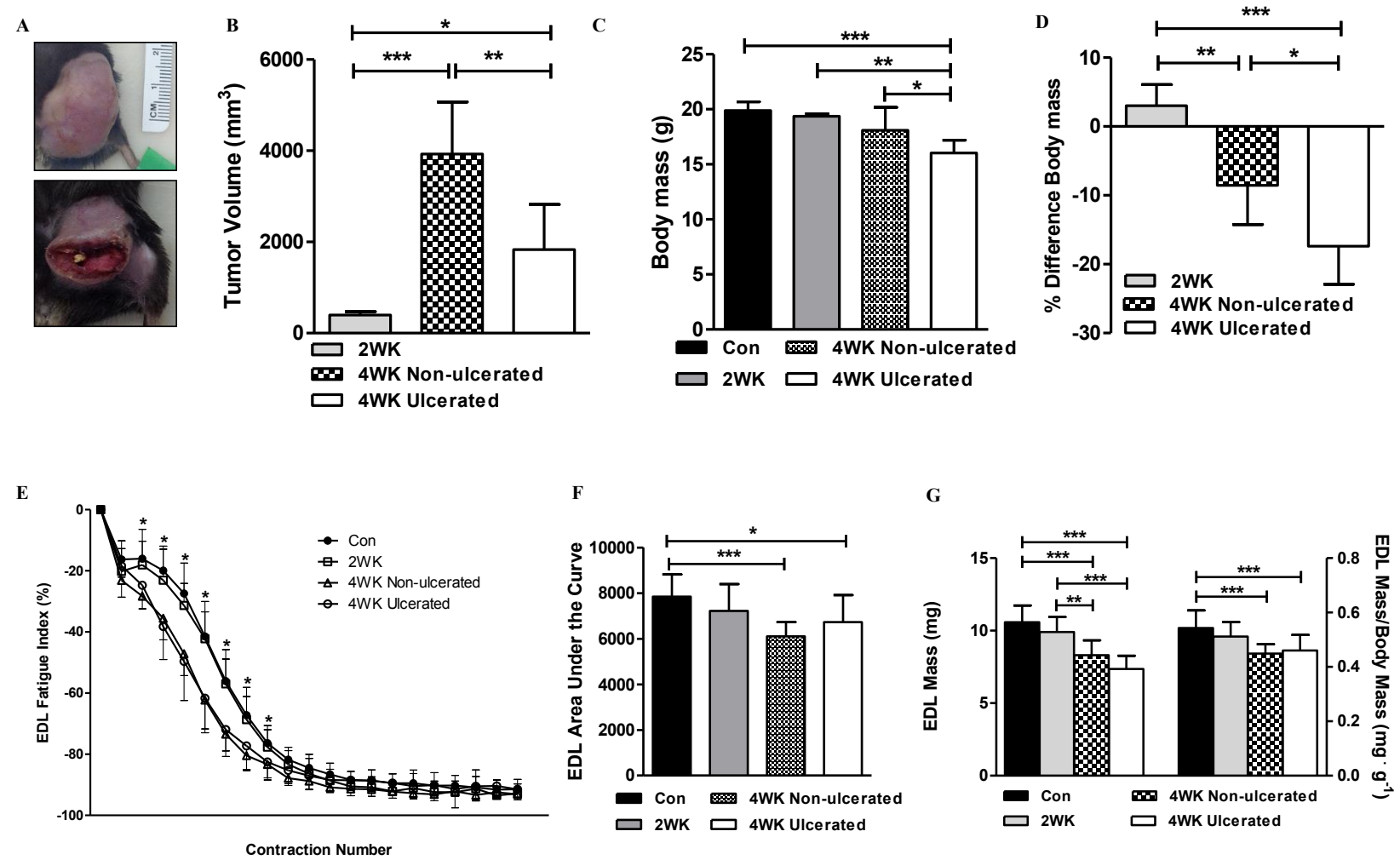

(A) Following 4 weeks, tumors presented with a mixture of non-ulcerated and ulcerated tumors. (B) Tumor volume progressively increased through 4 weeks of growth, with non-ulcerated tumors being larger than ulcerated tumors at the time of euthanasia. (C) Body mass at euthanasia, following the removal of the mammary tumor, was significantly different in the mice with ulcerated tumors compared to all other groups. 
(D) Mice with ulcerated tumors lost a greater percentage of body mass through the 4 weeks of tumor growth, compared to all other groups. (E) The fatigue index curves of EDL muscles from mice with a non-ulcerated tumor and an ulcerated tumor were shifted down and to the left compared to fatigue index curves of EDL muscles from control mice and mice following 2 weeks of tumor growth, demonstrating greater muscle fatigability. (F) The area under the EDL fatigue curve, indicative of the total amount of force produced during the fatigue protocol, was less in mice with a non-ulcerated tumor and an ulcerated tumor compared to control mice. (G) EDL absolute muscle mass and mass normalized to body mass. ${ }^{*}, p<0.05 ;{ }^{* *}, p<0.001 ;{ }^{* * *}, p<0.0001$. 
Figure 4: Causal mediation analysis in E0771 tumor-bearing mice.

Figure 4

$\mathbf{A}$

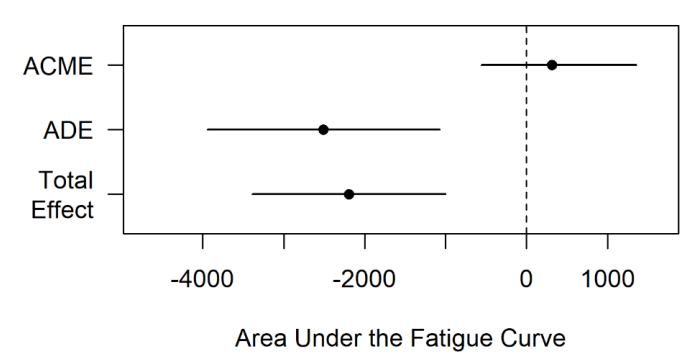

C

Control vs Tumor

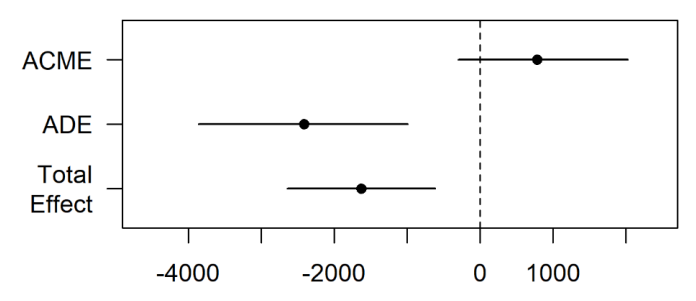

Area Under the Fatigue Curve

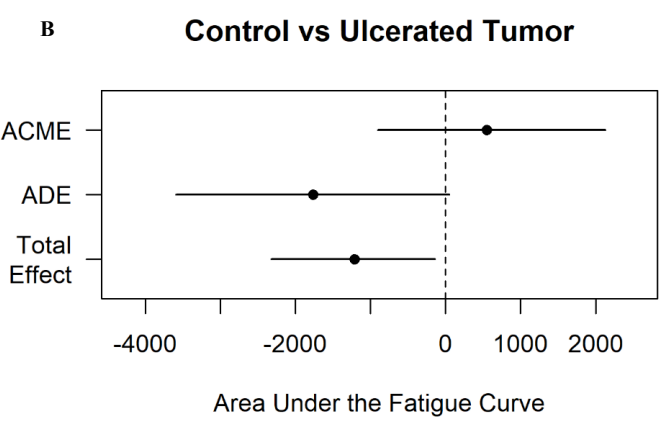

D Control vs Tumor, Tumor Ulceration Covariate

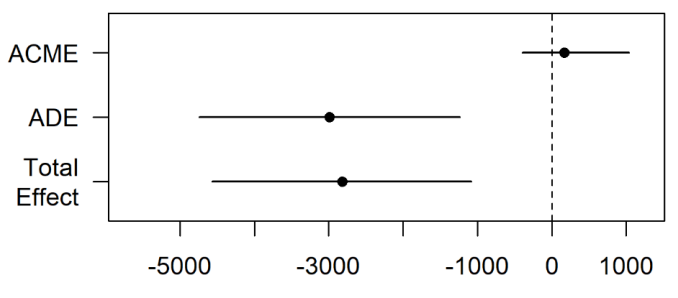

Area Under the Fatigue Curve

Graphical representation of the estimate and $95 \%$ confidence interval for the Total Effect, the Average Direct Effect (ADE) and the Average Causal Mediation Effect (ACME) for all CMA models tested. The direct effect of tumor growth on muscle fatigue was highly correlated with the total effect, while there was no significant mediator effect of muscle mass in comparing control mice to mice with non-ulcerated tumors $(\mathbf{A})$ or mice with ulcerated tumors (B). Similar results were obtained when both tumor groups were combined into a single group for comparison to control (C), and when the presence of tumor ulceration was used as a covariate in the analysis (D). Data presented as the mean and $95 \%$ confidence intervals for the ADE and ACME tested in these models. 
TABLES

Table 1: Summary of Causal Mediation Analysis Models

\begin{tabular}{|c|c|c|c|c|c|}
\hline Model & Comparison & $\begin{array}{c}\text { ACME Estimate } \\
\text { (Muscle Mass) } \\
(95 \% \mathrm{Cl})\end{array}$ & $\begin{array}{l}\text { ACME } \\
\text { p-value }\end{array}$ & $\begin{array}{l}\text { ADE Estimate } \\
\text { (Fatigue) } \\
(95 \% \mathrm{Cl})\end{array}$ & $\begin{array}{c}\text { ADE } \\
\text { p-value }\end{array}$ \\
\hline \multirow[t]{4}{*}{$\begin{array}{l}\text { BC- } \\
\text { PDOX }\end{array}$} & $\begin{array}{l}\text { All Control vs. PDOX } \\
\text { (age as covariate) }\end{array}$ & $-152(-667,176)$ & 0.41 & $-1660(-2490,-822)$ & $<0.0001^{* * *}$ \\
\hline & All Control vs. PDOX & $-150(-655,167)$ & 0.42 & $-1650(-2500,-831)$ & $<0.0001^{* * *}$ \\
\hline & Naïve Control vs. PDOX & $-147(-664,171)$ & 0.43 & $-2080(-2940,-1233)$ & $<0.0001^{* * *}$ \\
\hline & Surgical Control vs. PDOX & $-170(-717,156)$ & 0.38 & $-886(-1767,7)$ & 0.05 \\
\hline \multirow[t]{4}{*}{ E0771 } & $\begin{array}{l}\text { Control vs. All Tumor } \\
\text { (tumor ulceration as } \\
\text { covariate) }\end{array}$ & $168(-407,1053)$ & 0.61 & $-2990(-4760,-1226)$ & $<0.0001^{* * *}$ \\
\hline & Control vs. All Tumor & $782(-304,2041)$ & 0.16 & $-2403(-3855,-951)$ & $0.001^{* *}$ \\
\hline & Control vs. Ulcerated & $548(-928,2169)$ & 0.47 & $-1768(-3601,23)$ & 0.05 \\
\hline & Control vs. Non-ulcerated & $315(-600,1349)$ & 0.48 & $-2513(-3979,-194)$ & $0.001^{* *}$ \\
\hline & & & & & \\
\hline
\end{tabular}


CHAPTER 5: Breast cancer-associated skeletal muscle mitochondrial dysfunction and lipid accumulation is reversed by PPARG

The work described in the previous three chapters clearly implicates the peroxisomeproliferator activated receptors (PPARs) in the pathophysiology of skeletal muscle dysfunction in breast cancer (BC) and in animal models of BC. This work expands on those observations by transitioning to an in vitro model system to identify metabolic alterations induced in skeletal muscle directly by $\mathrm{BC}$-derived factors and to determine whether the PPAR proteins are indeed involved in the response. Here, we show unequivocally that $\mathrm{BC}$ cells secrete a factor that represses the transcriptional activity of PPARs, downregulation of PPARG target genes, mitochondrial dysfunction, and accumulation of lipid within differentiated muscle cells. The PPARG agonist drug rosiglitazone was able to rescue $\mathrm{BC}$-induced lipid accumulation, but did not rescue effects of BC-derived factors on PPAR-mediated transcription or mitochondrial function, but expression of exogenous PPARG protein reversed both BC-induced mitochondrial dysfunction and lipid accumulation. Collectively, these data indicate that BC-induced lipid metabolism and mitochondrial function occur via different mechanisms that are both related to PPAR signaling.

This article is almost entirely my independent work. I developed the protocols for the in vitro model system for use in our laboratory and independently collected all of the data presented herein. I was also the main contributor to the manuscript text.

This article is currently under review at Journal of Biological Chemistry as of April 10, 2020. 
Breast cancer-associated skeletal muscle mitochondrial dysfunction and lipid accumulation is reversed by PPARG

Hannah E. Wilson (1,2), David A. Stanton (3), Emidio E. Pistilli $(2,3,4,5)$

1. MD/PhD Medical Scientist Program, West Virginia University School of Medicine, Morgantown, WV, 26506.

2. Cancer Institute, West Virginia University School of Medicine, Morgantown, WV, 26506.

3. Division of Exercise Physiology, Department of Human Performance, West Virginia University School of Medicine, Morgantown, WV, 26506.

4. Department of Microbiology, Immunology, and Cell Biology, West Virginia University School of Medicine, Morgantown, WV, 26506.

5. West Virginia Clinical and Translational Sciences Institute, West Virginia University School of Medicine, Morgantown, WV, 26506.

${ }^{*}$ Corresponding Author:

Emidio E. Pistilli, PhD

West Virginia University School of Medicine

Morgantown, WV 26506

304-293-0291 (office) 304-293-7105 (fax)

epistilli2@hsc.wvu.edu

Acknowledgements:

This research was supported by the following: National Institute of General Medical Sciences of the National Institutes of Health under Award Number P20GM121322 (Lockman), American Cancer Society Institutional Research Grant 09-061-04 (Pistilli), the WVCTSI U54GM104942 (Hodder). Authors would like to acknowledge the following WVU Core Facilities for contributing to this work: Flow Cytometry and Single Cell Core Facility (S10OD016165); Mitochondria Core of the WVU Stroke CoBRE (P20GM109098); Mitochondria, Metabolism and Bioenergetics group (R01 HL-128485; Hollander and the Community Foundation for the Ohio Valley Whipkey Trust). Imaging experiments were performed in the West Virginia University Microscope Imaging Facility which has been supported by the WVU Cancer Institute, the WVU HSC Office of Research and Graduate Education, and NIH grants P20RR016440, P30GM103488 and P20GM103434. 


\section{ABSTRACT}

The peroxisome-proliferator activated receptors (PPARs) have been previously implicated in the pathophysiology of skeletal muscle dysfunction in women with breast cancer (BC) and in animal models of BC. Here, we sought to describe the metabolic alterations induced in skeletal muscle by BC-derived factors in an in vitro conditioned media (CM) system and hypothesized that $B C$ cells secrete a factor that represses PPAR-gamma (PPARG) expression and its transcriptional activity, leading to downregulation of PPARG target genes involved in mitochondrial function and other metabolic pathways. We found that BC-derived factors repress PPAR-mediated transcriptional activity without altering protein expression of PPARG. Further, we show that BC-derived factors induce significant alterations in skeletal muscle mitochondrial function and lipid metabolism, which are rescued with exogenous expression of PPARG. The PPARG agonist drug rosiglitazone was able to rescue BC-induced lipid accumulation, but did not rescue effects of BC-derived factors on PPAR-mediated transcription or mitochondrial function. These data suggest that BC-derived factors induce deficits in lipid metabolism and mitochondrial function via different mechanisms that are both related to PPARG signaling, with mitochondrial dysfunction likely being altered via repression of PPAR-mediated transcription, and lipid accumulation being altered via transcription-independent functions of PPARG.

Keywords: breast cancer, cancer-related fatigue, peroxisome-proliferator activated receptors, mitochondrial electron transport chain deficiencies, myosteatosis 


\section{INTRODUCTION}

Breast cancer (BC)-associated skeletal muscle fatigue is a chronic problem among $\mathrm{BC}$ survivors, being reported by a majority of patients both prior to and after receiving anticancer therapies ${ }^{1-5}$. Recent studies show that deficits in muscle function predict shorter survival in cancer, perhaps due to the fact that fatigue is known to reduce a patient's tolerance to anti-cancer therapies ${ }^{3,6-10}$. Therefore, improving muscle function in BC patients has the potential to improve both quality of life and survival in the most commonly diagnosed cancer type in women.

The peroxisome-proliferator activated receptors (PPARs) are lipid sensing, ligand activated transcription factors previously implicated in a variety of pathologies, including cancer, atherosclerosis, Alzheimer's disease, type 2 diabetes mellitus, and others ${ }^{11-14}$. These transcription factors aid in regulating whole body energy homeostasis via regulation of genes involved in lipid metabolism and mitochondrial functions ${ }^{11,15-19}$. Recently, downregulation of PPAR-gamma's (PPARG) transcriptional activity in skeletal muscle has been identified as a potential central regulator of the increased muscle fatigue experienced by women with $\mathrm{BC}$ and recapitulated in the patient-derived orthotopic xenograft model of $B C^{20}$. However, the mechanism by which PPARG downregulation induces muscle dysfunction has not yet been explored. We hypothesized that BC cells secrete a factor that represses PPARG expression and its transcriptional activity, leading to downregulation of PPARG target genes involved in mitochondrial function and other metabolic pathways. 


\section{METHODS}

Cell culture. Cell lines utilized include EpH4-EV (immortalized normal murine mammary epithelium), EO771 (murine luminal BC), NF639 (murine HER2/neuoverexpressing BC), HEK293 (human embryonic kidney), and C2C12 (murine myoblasts). All cell lines were obtained from ATCC (Virginia, USA), with the exception of EO771, which were obtained from Dr. Metheny-Barlow at Wake Forest University. All cell lines were cultured in DMEM (Thermo Fisher, Massachusetts, USA) supplemented with $10 \%$ heat inactivated fetal bovine serum (Atlanta Biologicals, Georgia, USA) and penicillin/streptomycin (Thermo Fisher) at $37^{\circ} \mathrm{C}$ with $6 \% \mathrm{CO} 2$.

Exogenous PPARG myogenic cell line. Lentiviral particles containing pLenti-CPPARG2-mGFP-P2A-Puro or pLenti -mGFP-P2A-Puro were purchased from Origene (Maryland, USA). Titers were provided by the manufacturer. C2C12 cells were plated at 125,000 cells well $^{-1}$ in 24-well plates and infected with a multiplicity of infection of 75 transforming units per cell with $8 \mu \mathrm{g} \cdot \mathrm{mL}^{-1}$ polybrene in antibiotic-free DMEM. Media was changed 20 hours after infection. Starting 48 hours after infection, cells were cultured in $2 \mu \mathrm{g} \cdot \mathrm{mL}^{-1}$ puromycin for 10 days, after which cells were maintained in 0.5 $\mu \mathrm{g} \cdot \mathrm{mL}^{-1}$ puromycin indefinitely. Uninfected control cells exhibited $100 \%$ death within 3 days of puromycin selection. Second passage cells were used in Western blotting analysis to assess expression of PPARG2 protein. All experiments with these cell lines were conducted within 5 passages of lentiviral infection as higher passage cells lost differentiation competence. 
Conditioned media (CM) collection. CM donor cells were plated at approximately $15 \%$ confluence in separate $10 \mathrm{~cm}$ dishes for 48 hours. The 48 -hour CM was then removed from all cell lines using a serological pipette, centrifuged at 1,500 RPM for 10 minutes, and the supernatants were collected via decanting into new centrifuge tubes. The collected CM was then diluted 1:3 in fresh growth media prior to application to recipient cells. CM was always applied to recipient cells within 2 hours of collection, in most cases within 15 minutes of collection (Figure 1A). In some experiments, rosiglitazone (Millipore-Sigma, Massachusetts, USA) in dimethyl sulfoxide (DMSO) was added to the diluted conditioned media to final concentrations of $10 \mu \mathrm{M}$ rosiglitazone and $0.1 \%$ DMSO prior to CM application to donor cells. In experiments where rosiglitazone was used, DMSO was added to control $\mathrm{CM}$ to $0.1 \%$ final concentration as a vehicle control.

Western blotting. Differentiated $\mathrm{C} 2 \mathrm{C} 12 \mathrm{~s}$ were lysed in $50 \mathrm{mM}$ Tris- $\mathrm{HCl}(\mathrm{pH} 6.8)$ with $2 \%$ sodium dodecyl sulfate and heated to $100^{\circ} \mathrm{C}$ for 3 cycles of 3 minutes each with vortexing and brief centrifugation between heating cycles. Lysates were diluted to a final concentration of $1 \mu \mathrm{g} \cdot \mathrm{LL}^{-1}$ in NuPAGE LDS Sample Buffer (ThermoFisher Scientific) and $5 \% \beta$-mercaptoethanol. $12 \mu \mathrm{g}$ of total protein was loaded per well and resolved in NuPAGE Novex 4-12\% Bis-Tris Gels (ThermoFisher Scientific). Proteins were transferred to nitrocellulose membrane, blocked for 1 hour in $1 \mathrm{X}$ tris-buffered saline (TBS), $0.1 \%$ Tween $20,5 \%$ milk followed by incubation with primary antibody overnight at $4^{\circ} \mathrm{C}$ in TBS-tween 20 with $5 \%$ milk. Membranes were then washed 3 times in TBS + $0.1 \%$ Tween20 prior to application of appropriate secondary antibody (ThermoFisher Scientific) for 90 minutes at room temperature, and again prior to application of Pierce 
ECL Western Blotting Substrate (ThermoFisher Scientific). Relative band intensity was quantified using the GE Amersham Imager 600 (GE Healthcare Life Sciences, Marlborough, MA, USA) and normalized to GAPDH. Primary antibodies included PPARY (\#PA3-821A) and GAPDH (\#2118S).

CM metabolic analyses. C2C12 cells were plated into Agilent Seahorse XF96 (Agilent Technologies, California, USA) plates at 10,000 cells well $^{-1}$ and differentiated in $2 \%$ horse serum (Atlanta Biologicals) in DMEM with antibiotics for 48 hours. Meanwhile, EpH4-EV, EO771, NF639, and C2C12 cells were plated at approximately $15 \%$ confluence in separate $10 \mathrm{~cm}$ dishes for 48 hours. 48 -hour CM, prepared as described above, was then applied to the 2-day differentiated $\mathrm{C} 2 \mathrm{C} 12$ cells in Seahorse assay plates for 48 hours ( $n=6-12$ wells per treatment condition, noted in figure legends) prior to conducting the Agilent Seahorse XF Cell Mito Stress Test or Agilent Seahorse XF Glycolysis Stress Test protocol according to manufacturer's instructions, using manufacturer's recommended reagents.

PPAR-reporter assays. HEK293 cells were transfected with PPRE-H2b-eGFP ${ }^{21}$ (Addgene \#84393) and selected for stable construct expression to create the HEK293PPRE-H2b-eGFP (HEK-PGFP) cell line. CM donor cells were plated on D0 as described above, and HEK-PGFP cells were plated at approximately $15 \%$ confluence on Day 1 (D1). On D2, baseline GFP intensity was collected using the BioTek Cytation 5 Cell Imaging Multi-Mode Reader (Agilent Technologies), and subsequently 48-hour conditioned media from HEK293, EpH4-EV, EO771, and NF639 cell lines was applied to the 24-well plate ( $n=3-6$ wells per treatment condition, noted in figure legends). HEKPGFP cells were then incubated in CM under normal culture conditions and GFP 
intensity was quantified 24 hours later using identical imaging settings as the baseline measurements. Mean GFP intensity per well was calculated using Gen5 Microplate Reader and Imager Software (Agilent Technologies), obtaining a single value per well that represented the mean GFP intensity of all cells in the imaging field for that well.

qRT-PCR for PPAR target genes. C2C12 cells were plated at approximately $90 \%$ confluence in 24 -well plates $\left(150,000\right.$ cells well $\left.^{-1}\right)$ on D0 and differentiated in $2 \%$ horse serum (Atlanta Biologicals) in DMEM with antibiotics. On D3, differentiation media was refreshed on the $\mathrm{C} 2 \mathrm{C} 12 \mathrm{~s}$, and $\mathrm{CM}$ donor cells were plated as described under " $\mathrm{CM}$ collection." On D4, media on the differentiating $\mathrm{C} 2 \mathrm{C} 12 \mathrm{~s}$ was changed back to normal growth media (10\% FBS in DMEM with antibiotics). On D5, CM was collected and applied to the differentiated $\mathrm{C} 2 \mathrm{C} 12$ cells for 2 or 24 hours, with $n=3-8$ wells per condition, as noted in figure legends. Total RNA was isolated from CM- and/or drugtreated cells using Trizol (ThermoFischer Scientific, Waltham, MA, USA) and established methods ${ }^{22} .1200 \mu \mathrm{g}$ of cDNA was produced using Invitrogen SuperScript III First-Strand Synthesis System (ThermoFisher Scientific) according to manufacturer's protocol, and relative expression of selected genes was analyzed using SYBR Green PCR Master Mix (ThermoFisher Scientific) with the Applied Biosystems QuantStudio 6 Flex (ThermoFisher Scientific), using 60 ng cDNA per reaction. Primer efficiencies were determined to be between $85 \%$ and $115 \%$ and relative mRNA expression was calculated using the Pfaffl method ${ }^{23}$ normalized to $18 \mathrm{~s}$ rRNA. Primer3 was used to design qRT-PCR primers (Supplementary Table 1) ${ }^{24}$. 
qRT-PCR for mitochondrial DNA. C2C12 cells were plated at approximately $90 \%$ confluence in 24-well plates $\left(100,000\right.$ cells.well $\left.{ }^{-1}\right)$ on D0 and differentiated in $2 \%$ horse serum in Gibco DMEM with antibiotics. Differentiation and CM application was conducted as described under "qRT-PCR for PPAR target genes," with CM applied for 24 hours and $n=8$ wells per condition. Total DNA was isolated from CM-treated cells using the DNeasy Blood \& Tissue Kit (Qiagen, Hilden, Germany) according to manufacturer's instructions. 40-60 ng of DNA in 1uL eluting buffer was used for qRTPCR using Applied Biosystemcs TaqMan Universal PCR Master Mix (ThermoFisher Scientific) with the Applied Biosystems QuantStudio 6 Flex (ThermoFisher Scientific). Primer-probe sets were purchased from ThermoFisher Scientific (Applied Biosystems Gene Expression Assays, COX2 Mm03294838_g1 and 18S Mm03928990_g1). Relative COX2 mtDNA was quantified using the $\triangle \Delta C T$ method normalized to $18 \mathrm{~S}$ gDNA.

Oil red O. C2C12 cells were plated at approximately $90 \%$ confluence in 24 -well plates $\left(100,000\right.$ cells well $\left.^{-1}\right)$ on D0 and differentiated in $2 \%$ horse serum in Gibco DMEM with antibiotics. On D2, differentiation media was refreshed on the $\mathrm{C} 2 \mathrm{C} 12 \mathrm{~s}$, and $\mathrm{CM}$ donor plates were set up as described under "CM collection." On D4, CM was collected and applied to differentiated $\mathrm{C} 2 \mathrm{C} 12 \mathrm{~s}$, and $\mathrm{CM}$ donor cells were split to allow for $\mathrm{CM}$ to be collected once more after 48 hours incubation. On D6, CM was again collected and was used to refresh the $\mathrm{CM}$ on the recipient cells. On D7, cells were fixed in $4 \%$ formaldehyde for 30 minutes, washed 3 times in distilled water, and made permeable with $60 \%$ isopropanol for 10 minutes. Oil red o stock solution had been previously suspended in $100 \%$ isopropanol and filtered using a $0.2 \mu \mathrm{m}$ bottle-top filter. Oil red o 
stock solution was diluted to $60 \%$ in distilled water to create the working solution. Working solution was applied to the fixed and permeable cells for 10 minutes. Cells were washed $3 \mathrm{x}$ with distilled water and stored at $4^{\circ} \mathrm{C}$ until imaging, typically within 24 hours of staining. Images were taken using a Zeiss Axiovert 40 CFL microscope with a Zeiss Axiocam 105 color camera, running ZEN 2.3 software. Effort was made to take images at the center of each well to produce even illumination across images. All images within each experiment were taken using identical imaging settings. Images were analyzed using the countcolors package version $0.9 .1^{25}$ in $\mathrm{R}$ version $3.6 .1^{26}$, quantifying the area of each image within a $50 \%$ radius from true red.

Statistical analyses, reproducibility, and rigor. Data analyses were conducted in $\mathrm{R}$ version 3.6.1 ${ }^{26}$ using ggpubr version $0.2 .4{ }^{27}$ for data visualization and most statistical tests. Unless otherwise stated, all statistical tests used were unpaired, two-tailed t-tests for two-group comparisons and one-way ANOVA for multi-group comparisons. When multiple comparisons were used within an experiment, Holm-Bonferroni correction was applied to adjust $p$-values. Graphs represent data from a single, independent experiment. All experiments have been repeated at least twice with similar results, with most experiments being repeated more than 3 times. For all box and whisker plots: the total height of the box represent the interquartile range, the thick center line represents the median value, and the whiskers extend to the most extreme data point that is not an outlier. Outlier values are represented by a single dot beyond the whiskers. 


\section{RESULTS}

\section{BC-CM represses PPARG expression and PPAR-mediated transcription}

Because PPARG is downregulated in the skeletal muscle of women with $B C$ and in mouse models of $B C^{20}$, we first investigated the capacity of $\mathrm{BC}$-derived factors to downregulate Pparg mRNA expression in an in vitro model system. To test this, we collected $\mathrm{CM}$ from $\mathrm{BC}$ or control cell lines and applied this $\mathrm{CM}$ to differentiated skeletal muscle cells in culture (Figure 1A). Cells and large debris were removed from the media via gentle centrifugation and decanting, and the conditioned media was diluted in $1: 3$ in fresh growth media to correct $\mathrm{pH}$ and overall nutrient content. Importantly, all $\mathrm{CM}$ was applied to myotubes within 2 hours of collection to ensure that biologically active components would not degrade or be damaged by freezing prior to application. Using this model system, we found that $\mathrm{CM}$ from $\mathrm{BC}$ cell lines significantly downregulated Pparg mRNA expression (Figure 1B) and several of its purported transcriptional targets 28 in differentiated skeletal muscle after as little as two hours of CM exposure, compared to media conditioned by skeletal muscle myoblasts (Figure 1C). However, no significant change in PPARG protein expression was detected (Figure 1D), suggesting that the downregulation of PPARG target genes' mRNA is due to a repression of PPARmediated transcriptional activity, rather than a down-regulation of PPARG protein abundance. To directly address this discrepancy, we generated a reporter cell line stably expressing a PPAR-responsive reporter construct driving expression of GFP. Using this system, we have consistently observed a significant reduction in GFP intensity in response to $\mathrm{BC}-\mathrm{CM}$, compared to $\mathrm{CM}$ from the reporter cells themselves. In 
contrast, CM from a non-tumorigenic mammary epithelial cell line does not repress GFP expression (Figure 1E).

\section{BC-CM induces mitochondrial dysfunction and defects in lipid metabolism}

We next asked whether BC-CM induced functional alterations in differentiated muscle cells that could be reflective of the increased muscle fatigability seen in women with $B C$ and $\mathrm{BC}$ animal models. We found that $\mathrm{BC}-\mathrm{CM}$ induced substantial changes in aerobic metabolism (Figure 2A), specifically leading to a reduction of oxygen consumed in ATP generation (Figure 2B) with inconsistent or negative results in other aerobic parameters across 5 independent experiments (not shown). CM from the luminal EO771 BC cell line repressed aerobic metabolism without altering glycolytic function, while the HER2overexpressing NF639 line repressed both aerobic metabolism and glycolytic functions (Figure 2C). This reduction in aerobic metabolism does not appear to be related to mitochondrial biogenesis, as suggested by no alteration in mitochondrial DNA copy number in CM-treated myotubes (Figure 2D).

Because lipotoxicity-induced mitochondrial dysfunction has been reported in various clinical contexts ${ }^{29-32}$, and because individuals with cancer have been shown to have increased lipid content in muscle ${ }^{33,34}$, we assessed intramyocellular lipid concentration in response to $\mathrm{BC}-\mathrm{CM}$ using oil red o staining and automated image quantification. Using this methodology, we repeatedly observed a significant increase in intramyocellular lipid staining with EO771-CM, but in no experiment did we observe an increase in response to NF639-CM (Figures $2 \mathrm{E}-\mathbf{2 F}$ ). 


\section{Exogenous PPARG expression rescues BC-CM-induced mitochondrial dysfunction and lipid accumulation}

To determine whether or not the metabolic alterations induced in skeletal muscle by BCderived factors are regulated by PPARG, we first attempted to generate stable myogenic cell lines expressing exogenous PPARG constructs or shRNA against PPARG using lentiviral infection and subsequent selection. Consistent with previous reports ${ }^{35}$, genetic modification of PPARG in $\mathrm{C} 2 \mathrm{C} 12$ myoblasts resulted in cell lines that rapidly lost differentiation competence, within five passages from lentiviral infection. Despite these limitations, early passage cells expressing exogenous PPARG (Figure 3A) were used to ascertain whether PPARG is involved in the response of myotubes to BC-derived factors. In these experiments, it was found that cells expressing exogenous PPARG were resistant to both BC-CM-induced repression of aerobic ATP generation (Figure 3B) as well as BC-CM-induced lipid accumulation (Figures 3C - 3D). Cells stably expressing shRNA against PPARG lost differentiation capacity immediately after lentiviral infection and could therefore not be used to determine if PPARG ablation phenocopies the effect of BC-CM in the context of differentiated muscle cells.

\section{PPARG agonist rosiglitazone rescues BC-CM-induced lipid accumulation but fails to rescue $\mathrm{BC}-\mathrm{CM}$-induced mitochondrial dysfunction and PPAR repression} Finally, we pharmacologically targeted PPARG to overcome BC-induced mitochondrial dysfunction and lipid accumulation, using the potent PPARG agonist rosiglitazone (rosi) of the thiazolidinedione (TZD) drug class. Rosiglitazone did induce PPAR-mediated transcription in the absence of BC-CM (Figure 4A), but it was unable to rescue BCCM's repression of PPAR-mediated transcription (Figure 4B). Unsurprisingly then, this 
agonist did not rescue BC-induced mitochondrial dysfunction (Figure 4C). However, rosiglitazone did potently repress BC-induced lipid accumulation (Figure 4D). These data indicate that the mechanism by which rosiglitazone prevents $\mathrm{BC}$-induced lipid accumulation is independent of PPARG's transcriptional activity, and that BC-induced lipid accumulation is not the cause of BC-induced mitochondrial dysfunction in this model system. 


\section{DISCUSSION}

In the present study, we have identified that BC-derived factors can significantly alter metabolic function and PPAR activity in skeletal muscle cells without the involvement of immune or stromal cell mediators, indicating that $\mathrm{BC}$ cells secrete a factor that induces these effects. Numerous publications have characterized changes in serum contents of women with BC, including changes in miRNAs, lipids, and proteins ${ }^{36-40}$, though the exact sources of many of these factors remain unknown. To our knowledge, this report is the first showing that $\mathrm{BC}-\mathrm{CM}$ induces alterations in skeletal muscle metabolic function, with previous studies focusing on BC-CM's effect on immune cells, fibroblasts, endothelial cells, or normal mammary epithelium ${ }^{41-46}$, or on transcriptional changes induced in skeletal muscle ${ }^{47}$.

It is well established that $\mathrm{BC}$ cells can regulate both local and systemic immune functions ${ }^{48,49}$, and that inflammatory signaling is intrinsically linked to cancer-associated skeletal muscle wasting ${ }^{50,51}$. Thus, a reasonable assumption is that BC's influence on immune cells is the cause of BC-induced skeletal muscle fatigue. This study contradicts this assumption by showing that no immunological mediators are required for $\mathrm{BC}$ cells to induce significant alterations in skeletal muscle metabolic function. This study also contradicts recent reports of negative results in other cancer types, where CM from cancer cells was unable to directly induce mitochondrial deficits in differentiated skeletal muscle ${ }^{52}$, indicating that different cancer types induce skeletal muscle dysfunction via different mechanisms. While we cannot conclusively state that mitochondrial dysfunction or lipotoxicity are the root causes of BC-induced skeletal muscle dysfunction, it is reasonable to hypothesize a causal link that should be further 
investigated. Most likely, there are numerous mechanisms contributing to skeletal muscle dysfunction that would need to be simultaneously targeted to provide symptom control.

The mechanism by which BC cells induce metabolic dysfunction in this model system appears to be related to signaling by the PPAR proteins, as evidenced by the rescue of mitochondrial dysfunction and lipid content provided by exogenous expression of PPARG. This finding provides support for the translational relevance of this in vitro model system, as PPARG has been previously identified as a potential key regulator of $\mathrm{BC}$-induced skeletal muscle fatigue using both human data and data generated in the patient-derived orthotopic xenograft model of $B C^{20,53}$. Additionally, this finding illuminates potential therapeutic modalities using the numerous FDA-approved agents that target the PPAR proteins, including drugs with various specificities for the three PPAR isoforms. In support of this possibility, we show here that the PPARG-specific agonist rosiglitazone completely reversed BC-CM's effect on skeletal muscle lipid accumulation. Interestingly, this result does not appear to be due to rosiglitazone activating PPAR-mediated transcription, suggesting that the effect is mediated by noncanonical functions of PPARG.

While we were unable to rescue $\mathrm{BC}$-induced mitochondrial dysfunction using rosiglitazone, it is possible that pharmacological agents targeting PPAR-alpha, PPARdelta, or a combination of the three isoforms could overcome these effects of BCderived factors. Alternatively, it is possible that the mitochondrial dysfunction induced by BC-CM is due to alteration of ligand-independent functions of PPARG, which would be overcome by the addition of PPARG protein but not by exogenous ligand. Numerous 
reports confirm the ability of PPARG to function as a transcription factor in the absence of ligand binding ${ }^{54-57}$, and there is evidence of PPARG having ligand-independent functions that are also independent of its transactivation domain ${ }^{58}$. Pharmacological strategies to target these unique functions could include non-TZD insulin-sensitizing agents or epigenetic modifiers.

Mechanistically, muscle fatigue can result from a myriad of factors, but the underlying ability of the contractile elements to maintain force production relies upon the muscle's ability to resynthesize ATP following stimulus. We have observed dysregulation of metabolically significant PPAR transcriptional networks in the skeletal muscle of BCPDOX mice ${ }^{20}$, as well as a significant reduction in ATP from mitochondria isolated from skeletal muscle of these mice. In support of these murine observations, proteomics analysis in the skeletal muscle of HER2+ BC patients revealed lower protein expression of nearly every component of the mitochondrial electron transport chain and a correspondingly lower ATP content in muscle biopsies from BC-patients of multiple tumor subtypes (publication under review). These data clearly demonstrate mitochondrial dysfunction in muscle of patients with $\mathrm{BC}$, which will directly impact the fatigability of skeletal muscle. The results presented herein expand on our previous studies and suggest this metabolic dysfunction is a direct result of BC-derived factors. Intriguingly, we demonstrate that this mitochondrial dysfunction is accompanied by increased intracellular lipid deposition in myotubes exposed to BC-CM. Intramuscular lipid deposition has also been observed in type 2 diabetes ${ }^{59}$ and smoking-induced insulin resistance ${ }^{60}$. As the downregulation of PPARG expression and transcriptional activity is associated with the development of insulin resistance ${ }^{61}$ and many PPAR 
transcriptional targets are mitochondrial lipid transport proteins ${ }^{28}$, we propose that a decreased capacity for mitochondrial lipid import may contribute to the observed phenotype of BC-induced lipid accumulation, which may then contribute to insulin resistance. Finally, aberrant intramyocellular lipid deposition can in many cases contribute to lipotoxicity characterized by the accumulation of toxic lipid intermediates such as diacylglycerols and ceramides ${ }^{62}$, increased production of ROS ${ }^{63}$, and is associated with changes in autophagic dynamics ${ }^{64,65}$, all of which are implicated in reduced mitochondrial function within skeletal muscle. The sum of these observations suggest that skeletal muscle lipotoxicity in breast cancer could play a major role in the phenotype of BC induced skeletal muscle fatigue independent of mitochondrial dysfunction, and future studies should investigate this potential link in greater detail.

In summary, our results confirm the ability of BC-derived factors to directly alter metabolic function in skeletal muscle cells without the involvement of other cell types. These BC-CM-induced phenotypes in skeletal muscle could be enacted by a variety of secreted factors, including miRNAs, exosomes, or proteins, and appears to be mediated via signaling of the PPAR proteins, though perhaps not through their canonical functions as ligand-activated transcription factors. Specifically, BC-derived factors have the ability to repress the transcriptional activity of PPARG, and this effect is not due to downregulation of PPARG protein abundance. Additionally, repression of PPARG transcriptional activity induces mitochondrial dysfunction in muscle cells and a deficiency in ATP supply while also being associated with greater lipid accumulation. While PPARG protein levels are not affected in our model, basal protein expression of PPARG is relatively low in skeletal muscle, providing an explanation for the ability of 
exogenous PPARG to rescue these phenotypes while PPARG-agonists do not (Figure 5). These results provide support for previous publications implicating the PPAR proteins in $\mathrm{BC}$-induced skeletal muscle fatigue and provide rationale for investigating PPAR agonists to improve quality of life in survivors of $B C{ }^{20,53}$. 
FIGURES AND FIGURE LEGENDS

Figure 1: BC-CM represses PPARG expression and PPAR-mediated transcription

A
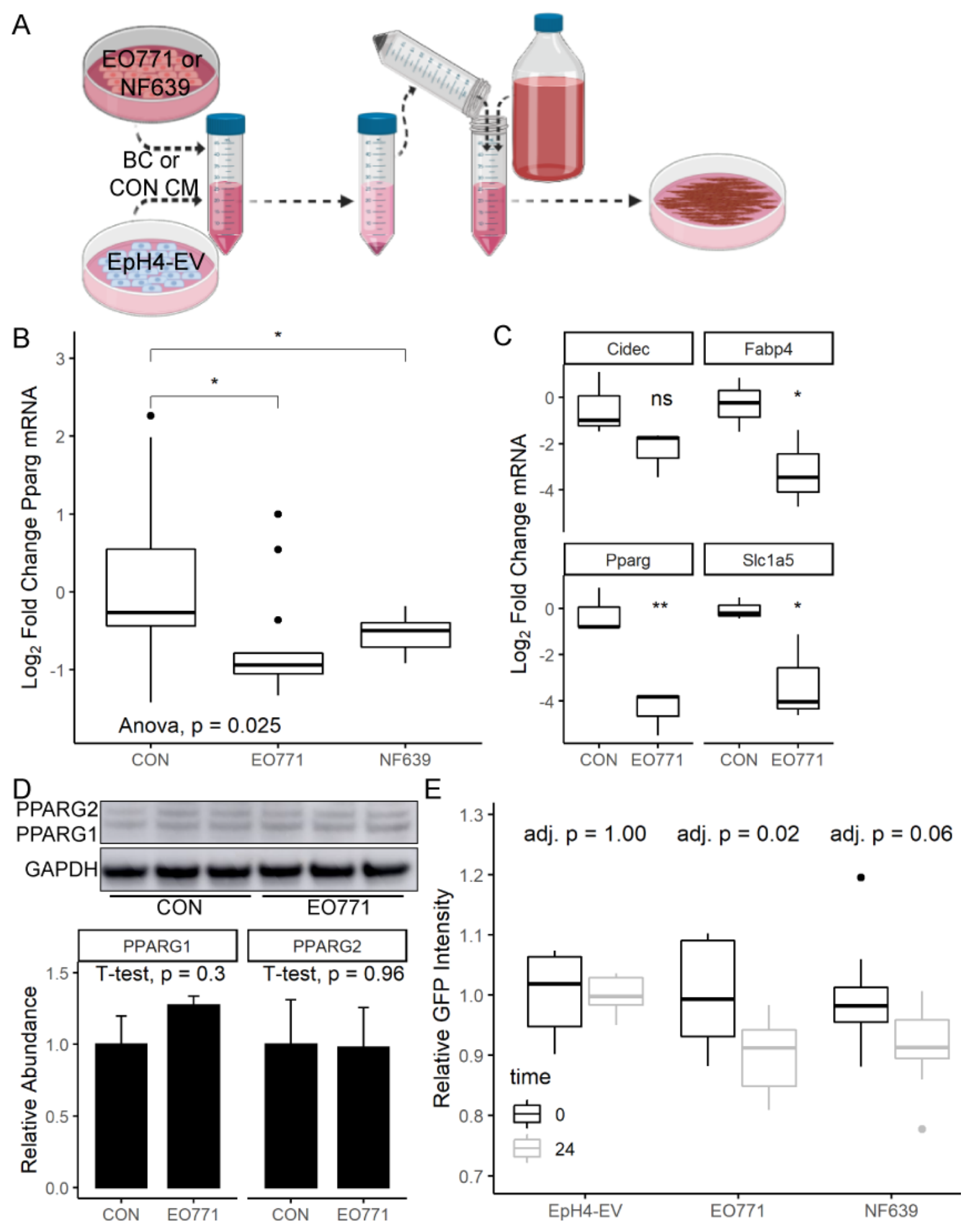
48-hour conditioned media was collected from BC (EO771 or NF639) or control cell lines (EpH4-EV, C2C12 or HEK293), centrifuged to pellet large debris, decanted, and diluted in fresh complete growth media prior to application on recipient cells. Figure created using BioRender (A). Pparg mRNA expression in 5 day differentiated C2C12s exposed to 48-hour CM for 24 hours, quantified using qRT-PCR, $\mathrm{n}_{\mathrm{CON}}=12, \mathrm{n}_{\mathrm{EO} 771}=6$, $\mathrm{n}_{\mathrm{NF} 639}=5(\mathrm{~B}) \cdot \mathrm{mRNA}$ expression of PPARG target genes in 5 day differentiated C2C12s exposed to 48-hour CM for 2 hours, quantified using qRT-PCR, $\mathrm{n}_{\mathrm{CON}}=3, \mathrm{n}_{\mathrm{EO} 771}=3(\mathrm{C})$. Western blotting analysis of PPARG protein expression in 5 day differentiated $\mathrm{C} 2 \mathrm{C} 12 \mathrm{~s}$ exposed to 48-hour CM for 48 hours, normalized to GAPDH. Error bars represent standard deviation of the mean (D). Quantification of GFP intensity in HEK-PGFP reporter cells exposed to 48-hour CM, quantified at 0 and 24 hours of CM exposure. Adjusted $p$-values represent Holm-Bonferroni adjusted $p$-values of paired t-tests, $n=8$ per group (E). 
Figure 2: BC-CM induces mitochondrial dysfunction and defects in lipid metabolism
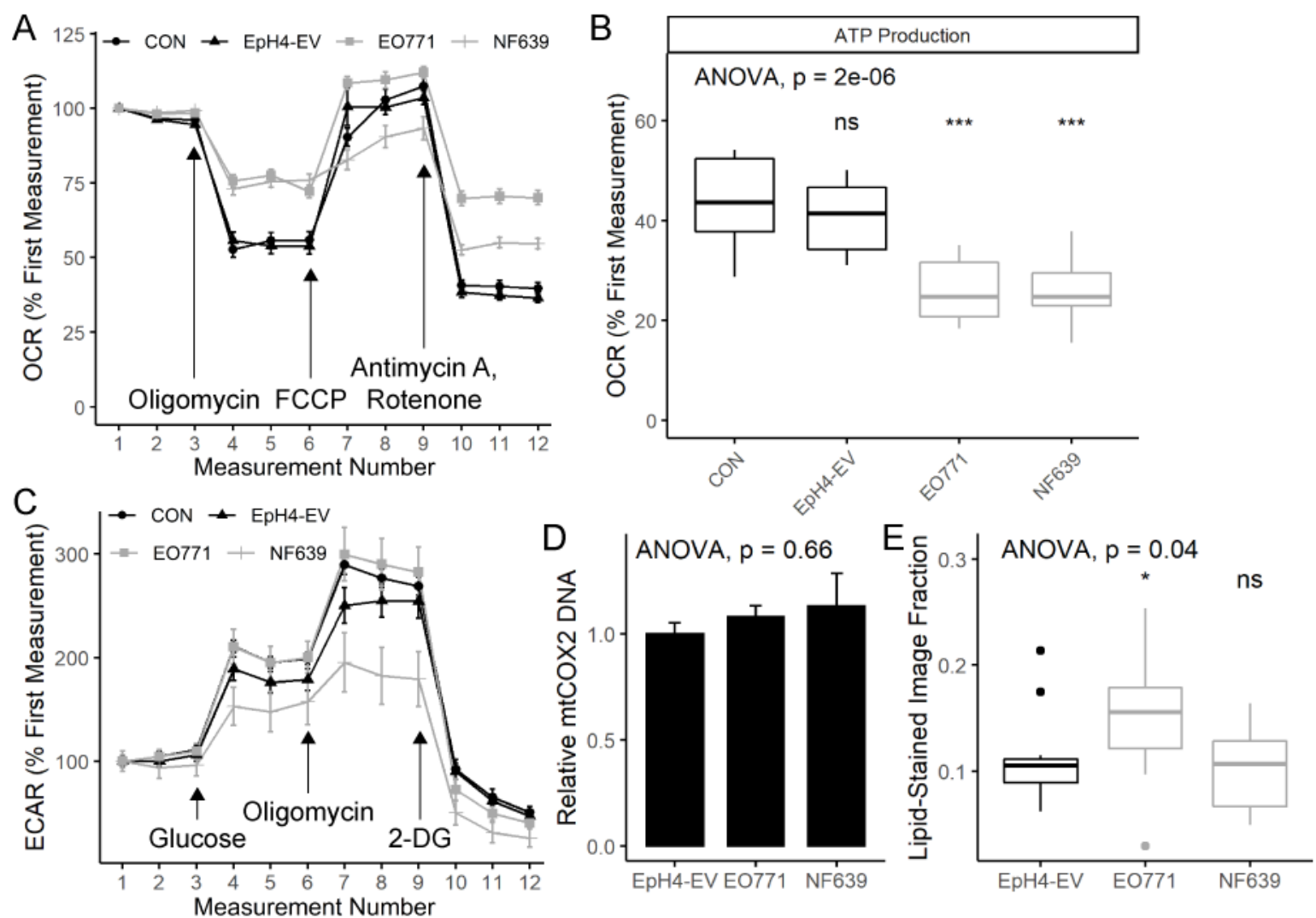

$\mathrm{F}$
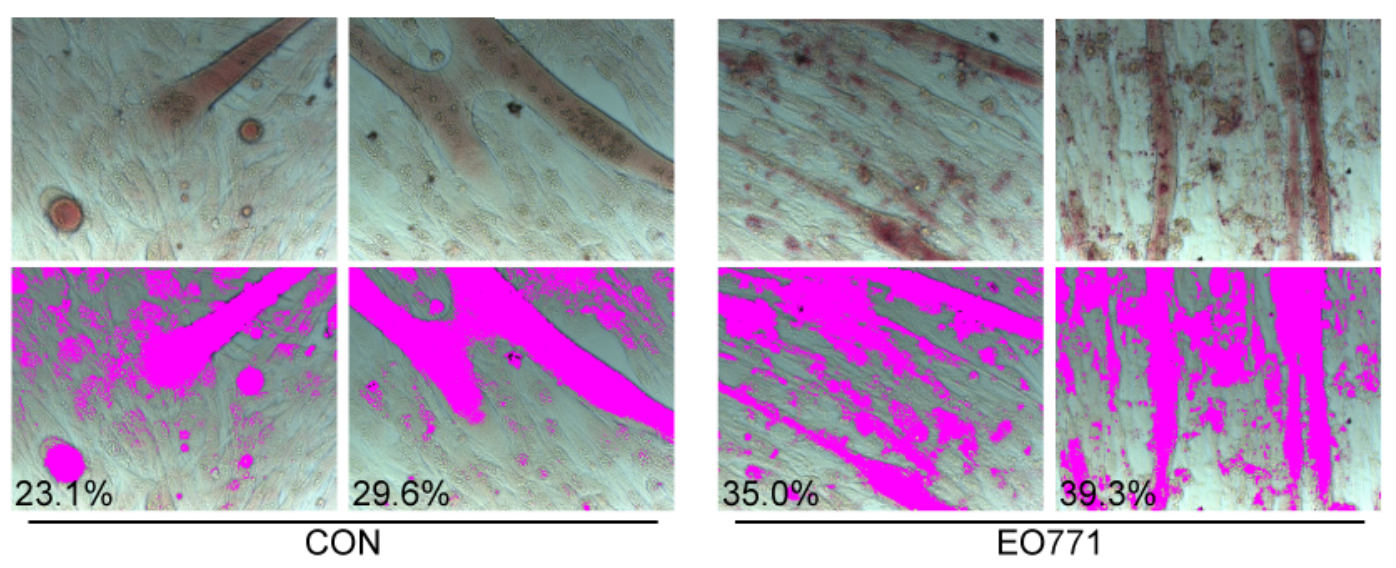

Normalized oxygen consumption rate (OCR) of 2 day differentiated $\mathrm{C} 2 \mathrm{C} 12$ myotubes treated with 48-hour CM for 48 hours, quantified using the Seahorse XF Mito Stress Test. Error bars represent standard deviation of the mean, $n=10$ per group (A). OCR associated with aerobic ATP production from A (B). Extra-cellular acidification rate of 2 
day differentiated $\mathrm{C} 2 \mathrm{C} 12$ myotubes treated with 48 -hour $\mathrm{CM}$ for 48 hours, quantified using the Seahorse XF Glycolysis Stress Test. Error bars represent standard deviation of the mean, $n=12$ per group, except $n_{N F 639}=10(C)$. Relative quantitation of mtCOX2 DNA in 5 day differentiated $\mathrm{C} 2 \mathrm{C} 12 \mathrm{~s}$ exposed to 48 -hour $\mathrm{CM}$ for 24 hours, normalized to 18S gDNA, $n=8$ per group (D). Oil red o image quantification from 4 day differentiated C2C12s treated with 48-hour CM for 72 hours, represented as the fraction of each image stained red with oil red $\mathrm{o}, \mathrm{n}=8$ per group $(\mathrm{E})$. Representative images from an independent oil red o experiment conducted under identical conditions to E, 40X magnification $(\mathbf{F})$. 
Figure 3: Exogenous PPARG expression rescues BC-CM-induced mitochondrial dysfunction and lipid accumulation

A

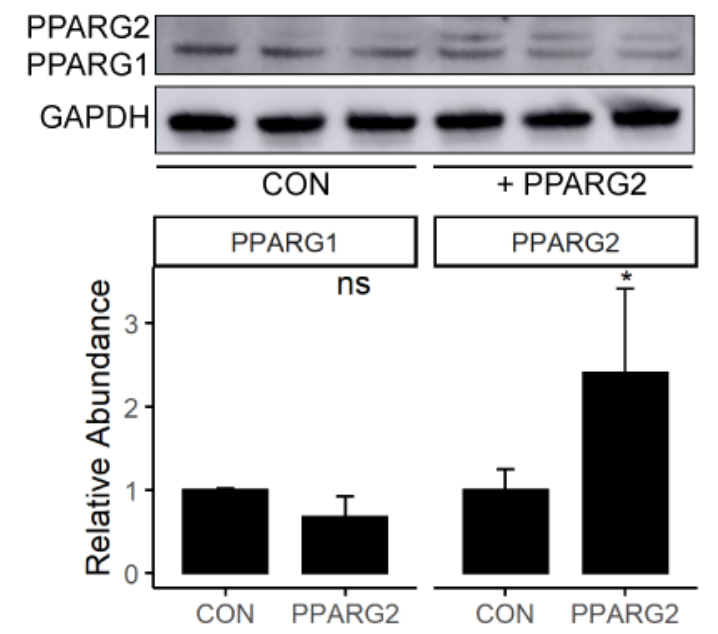

C

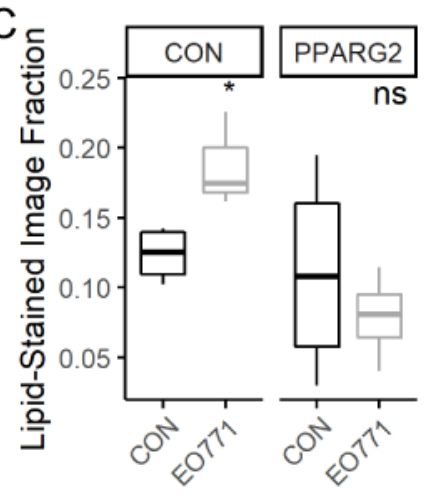

B

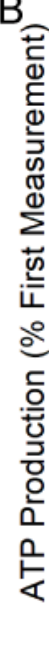

$\mathrm{D}$

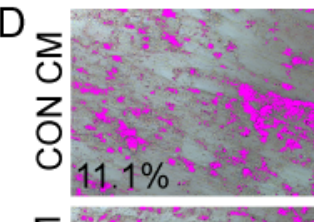

i

今

نำ1 16.1\%
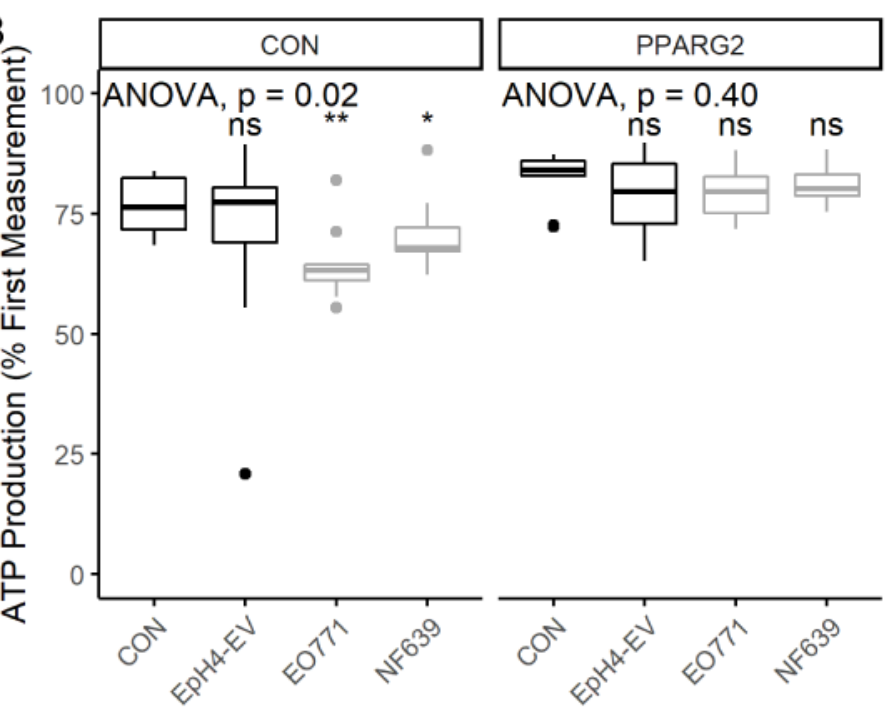

ANOVA, $p=0.40$
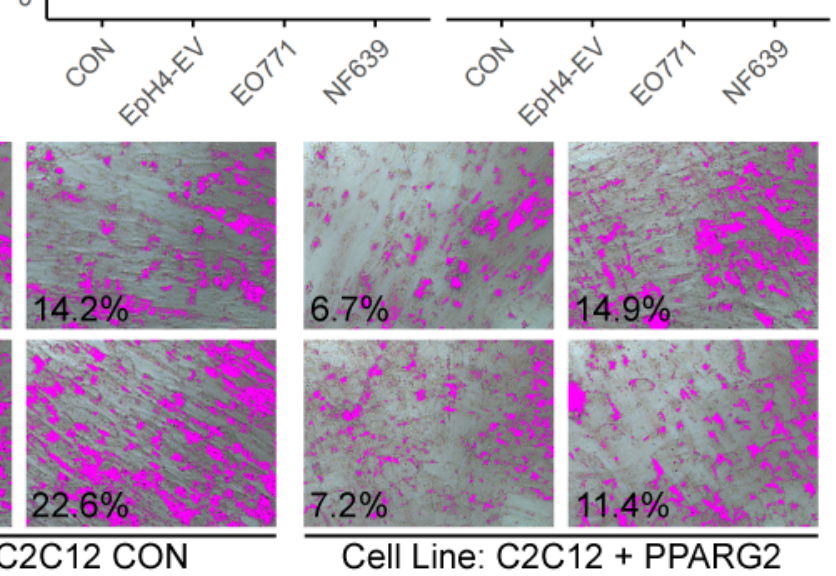

Western blotting analysis of PPARG expression in C2C12s infected with lentiviral particles carrying pLenti-C-PPARG2-mGFP-P2A-Puro (+PPARG2) or pLenti -mGFPP2A-Puro (CON) after 5 days of differentiation (A). OCR associated with aerobic ATP production in 2 day differentiated CON or +PPARG2 cells exposed to 48-hour CM for 48 hours, quantified using the Seahorse XF Mito Stress Test, $n=10$ per group (B). Oil red o image quantification from 4 day differentiated CON or +PPARG2 cells treated with 48hour CM for 72 hours, represented as the fraction of each image stained red with oil red 
$0, n=6$ per group (C). Representative images from the experiment quantified in $C, 20 X$ magnification (D). 
Figure 4: PPARG agonist rosiglitazone fails to rescue BC-CM-induced mitochondrial dysfunction and PPAR repression, but rescues BC-CM-induced lipid accumulation
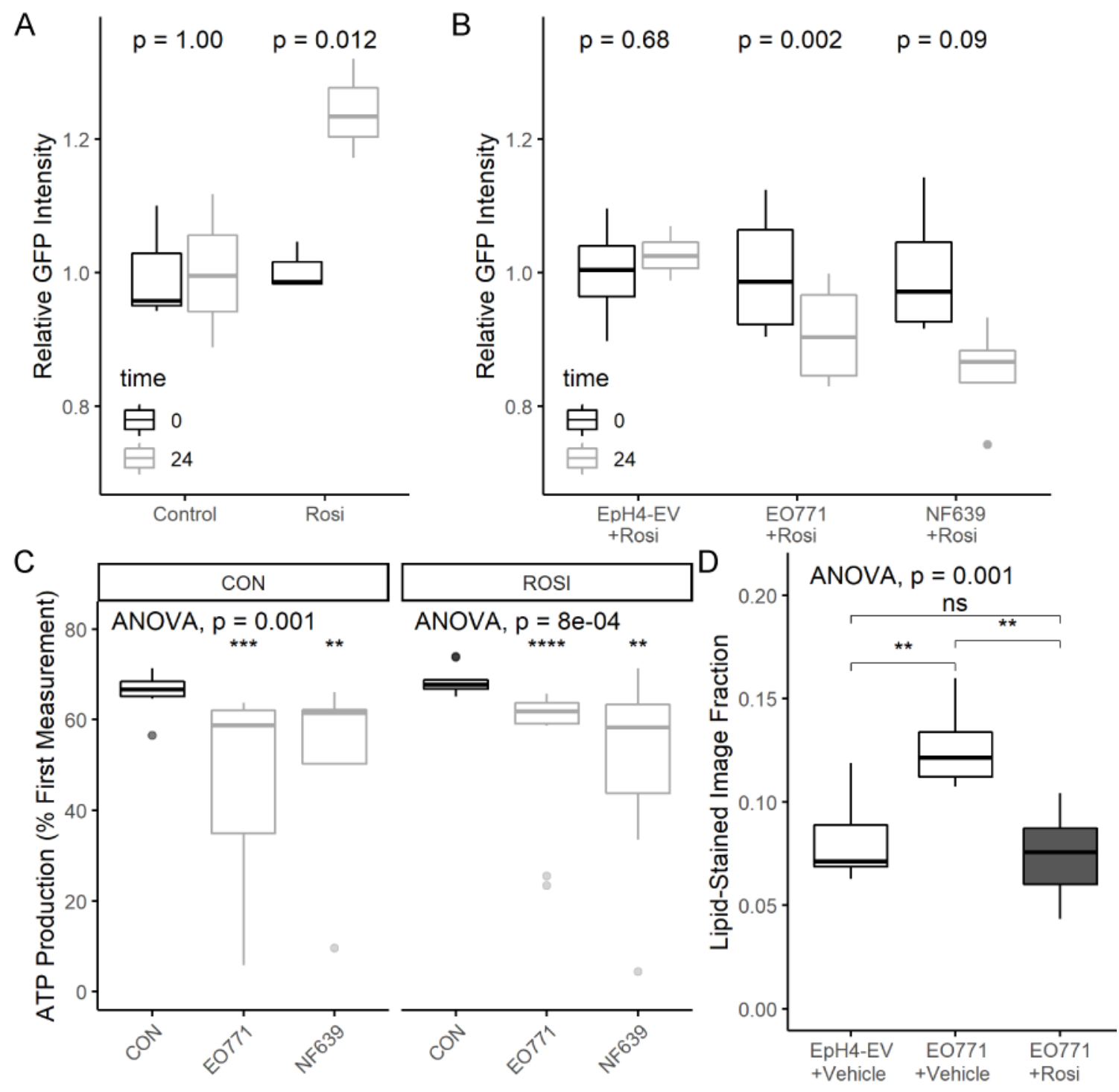

Quantification of GFP intensity in HEK-PGFP reporter cells exposed to $10 \mathrm{uM}$ rosiglitazone (rosi) or DMSO (control), quantified at 0 and 24 hours of drug exposure, $n$ = 3 per group (A). Quantification of GFP intensity in HEK-PGFP reporter cells exposed to 48 -hour $\mathrm{CM}$ in the presence of $10 \mu \mathrm{M}$ rosiglitazone, quantified at 0 and 24 hours of CM exposure. Adjusted $p$-values represent Holm-Bonferroni adjusted $p$-values of paired 
t-tests, $n=4$ per group (B). OCR associated with aerobic ATP production in 2 day differentiated $\mathrm{C} 2 \mathrm{C} 12 \mathrm{~s}$ cells exposed to 48 -hour $\mathrm{CM}+/$ - rosiglitazone for 48 hours, quantified using the Seahorse XF Mito Stress Test, $n=10$ per group, except ${ }_{n N F 639+r o s i}=$ 7 (C). Oil red o image quantification from 4 day differentiated $\mathrm{C} 2 \mathrm{C} 12$ cells treated with 48-hour CM for 72 hours, represented as the fraction of each image stained red with oil red $\mathrm{o}, \mathrm{n}=8$ per group (D). 
Figure 5: Summary of Findings

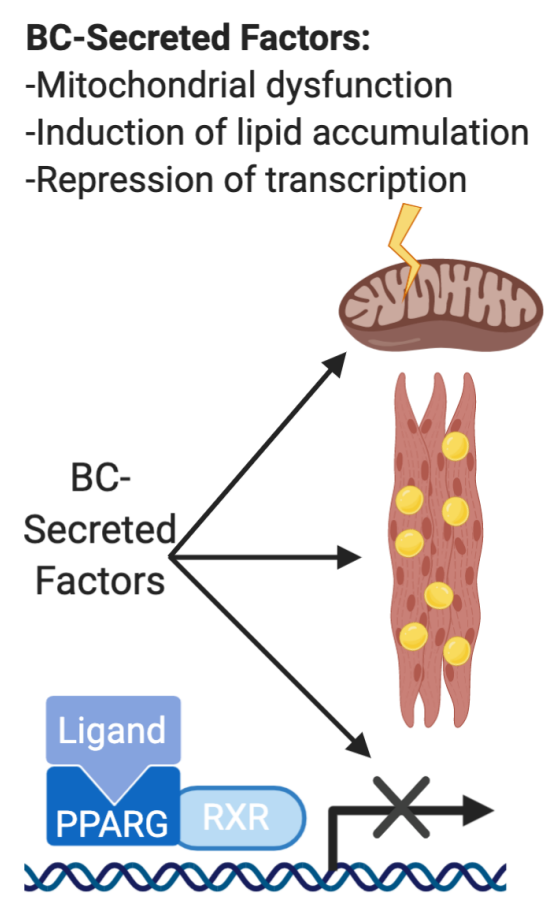

+Rosiglitazone:

-Mitochondrial dysfunction

-No lipid accumulation

-Repression of transcription

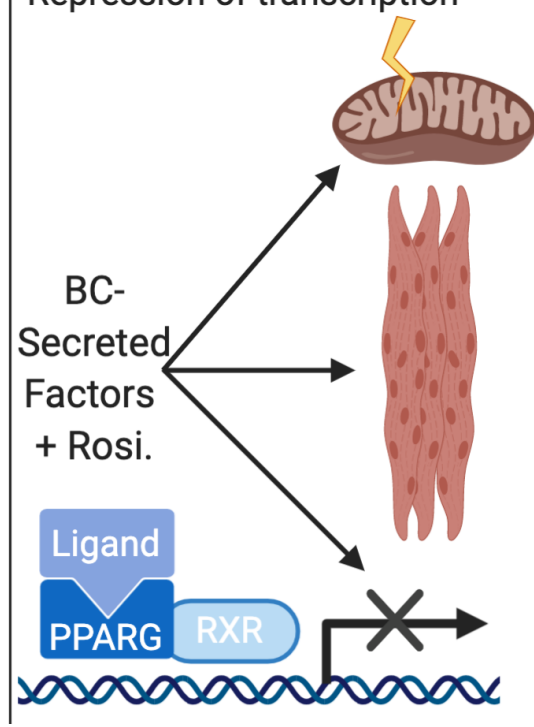

+Exogenous PPARG2:

-No mitochondrial dysfunction -No lipid accumulation

-Repression of transcription?

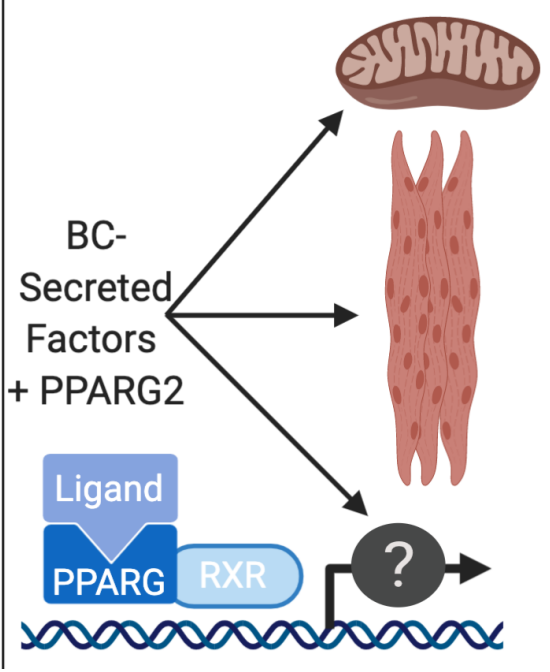

BC-derived factors induce several physiological changes in target cells in vitro, including mitochondrial dysfunction, lipid accumulation, and repression of PPAR-mediated transcription (left). The PPARG agonist Rosiglitazone reverses BC-induced lipid accumulation but does not reverse mitochondrial dysfunction or the observed repression of PPAR-mediated transcription (center). Expression of exogenous PPARG2 in myotubes reverses both BC-induced mitochondrial dysfunction and lipid accumulation. Figure created using BioRender (right). 
TABLES

Supplementary Table 1: Primers used for qRT-PCR

\begin{tabular}{|l|l|l|}
\hline & Forward Primer & Reverse Primer \\
\hline $18 s$ & 5'-AATGCTTTCGCTCTGGTCCG & 5'-CCTGGATACCGCAGCTAGGA \\
\hline Cidec & 5'-AGCTAGCCCTTTCCCAGAAG & 5'-CCTTGTAGCAGTGCAGGTCA \\
\hline Fabp4 & 5'-CATCAGCGTAAATGGGGATT & 5'-TCGACTTTCCATCCCACTTC \\
\hline Pparg & 5'-TGTGGGGATAAAGCATCAGGC & 5'-CCGGCAGTTAAGATCACACCTAT \\
\hline Slc1a5 & 5'-CATCACCATCCTGGTCACAG & 5'-CCTTCCACGTTGAGGACAGT \\
& \multicolumn{2}{|}{} \\
\hline
\end{tabular}




\section{REFERENCES}

1. Bower, J.E. et al. Fatigue in breast cancer survivors: occurrence, correlates, and impact on quality of life. J Clin Oncol 18, 743-53 (2000).

2. Cella, D., Lai, J.S., Chang, C.H., Peterman, A. \& Slavin, M. Fatigue in cancer patients compared with fatigue in the general United States population. Cancer 94, 528-38 (2002).

3. Curt, G.A. et al. Impact of cancer-related fatigue on the lives of patients: new findings from the Fatigue Coalition. Oncologist 5, 353-60 (2000).

4. Cella, D., Davis, K., Breitbart, W., Curt, G. \& Coalition, F. Cancer-related fatigue: prevalence of proposed diagnostic criteria in a United States sample of cancer survivors. J Clin Oncol 19, 3385-91 (2001).

5. Blesch, K.S. et al. Correlates of fatigue in people with breast or lung cancer. Oncol Nurs Forum 18, 81-7 (1991).

6. Arndt, V., Stegmaier, C., Ziegler, H. \& Brenner, H. A population-based study of the impact of specific symptoms on quality of life in women with breast cancer 1 year after diagnosis. Cancer 107, 2496-503 (2006).

7. Peters, K.B. et al. Impact of health-related quality of life and fatigue on survival of recurrent high-grade glioma patients. J Neurooncol 120, 499-506 (2014).

8. Wang, X.S. \& Woodruff, J.F. Cancer-related and treatment-related fatigue. Gynecol Oncol 136, 446-52 (2015). 
9. Groenvold, M. et al. Psychological distress and fatigue predicted recurrence and survival in primary breast cancer patients. Breast Cancer Res Treat 105, 209-19 (2007).

10. Prado, C.M. et al. Sarcopenia as a determinant of chemotherapy toxicity and time to tumor progression in metastatic breast cancer patients receiving capecitabine treatment. Clin Cancer Res 15, 2920-6 (2009).

11. Kersten, S., Desvergne, B. \& Wahli, W. Roles of PPARs in health and disease. Nature 405, 421-424 (2020).

12. Sabatino, L., Fucci, A., Pancione, M. \& Colantuoni, V. PPARG Epigenetic Deregulation and Its Role in Colorectal Tumorigenesis. PPAR Res 2012(2012).

13. Aronoff, S. et al. Pioglitazone hydrochloride monotherapy improves glycemic control in the treatment of patients with type 2 diabetes: a 6-month randomized placebo-controlled dose-response study. The Pioglitazone 001 Study Group. (2000).

14. Chandra, S. \& Pahan, K. Gemfibrozil, a Lipid-Lowering Drug, Lowers Amyloid Plaque Pathology and Enhances Memory in a Mouse Model of Alzheimer's Disease via Peroxisome Proliferator-Activated Receptor $\alpha$. (2019).

15. Lehrke, M. \& Lazar, M.A. The many faces of PPARgamma. Cell 123, 993-9 (2005).

16. Fan, W. \& Evans, R. PPARs and ERRs: molecular mediators of mitochondrial metabolism. Curr Opin Cell Biol 33, 49-54 (2015). 
17. Auwerx, J., Cock, T.A. \& Knouff, C. PPAR-gamma: a thrifty transcription factor. Nucl Recept Signal 1, e006 (2003).

18. Torra, I.P., Chinetti, G., Duval, C., Fruchart, J.C. \& Staels, B. Peroxisome proliferator-activated receptors: from transcriptional control to clinical practice. Curr Opin Lipidol 12, 245-54 (2001).

19. Berger, J. \& Moller, D.E. The mechanisms of action of PPARs. Annu Rev Med 53, 409-35 (2002).

20. Wilson, H.E. et al. Human breast cancer xenograft model implicates peroxisome proliferator-activated receptor signaling as driver of cancerinduced muscle fatigue. Clinical Cancer Research, clincanres.1565.2018 (2018).

21. Degrelle, S.A., Shoaito, H. \& Fournier, T. New Transcriptional Reporters to Quantify and Monitor PPARgamma Activity. PPAR Res 2017, 6139107 (2017).

22. Pistilli, E.E., Jackson, J.R. \& Alway, S.E. Death receptor-associated proapoptotic signaling in aged skeletal muscle. Apoptosis 11, 2115-26 (2006).

23. Pfaffl, M.W. A new mathematical model for relative quantification in real-time RT-PCR. Nucleic Acids Res 29, e45 (2001).

24. Untergasser, A. et al. Primer3--new capabilities and interfaces. Nucleic Acids Res 40, e115 (2012).

25. Weller, H. countcolors: Locates and Counts Pixels Within Color Range(s) in Images. (R, https://CRAN.R-project.org/package=countcolors, 2019). 
1. 26. R: A language and environment for statistical computing. (R Foundation for Statistical C omputing 2019).

26. Kassambara, A. ggpubr: 'ggplot2' Based Publication Ready Plots. (Comprehensive R Archive Network (CRAN), 2019).

27. Fang, L. et al. PPARgene: A Database of Experimentally Verified and Computationally Predicted PPAR Target Genes. PPAR Res 2016, 6042162 (2016).

28. Schrauwen, P., Schrauwen-Hinderling, V., Hoeks, J. \& Hesselink, M.K. Mitochondrial dysfunction and lipotoxicity. Biochim Biophys Acta 1801, 266$71(2010)$.

29. Kumar, B. et al. Lipotoxicity Augments Glucotoxicity-Induced Mitochondrial Damage in the Development of Diabetic Retinopathy. Investigative Ophthalmology \& Visual Science 56, 2985-2992 (2020).

30. Yang, L., Wei, J., Sheng, F. \& Li, P. Attenuation of Palmitic Acid-Induced Lipotoxicity by Chlorogenic Acid through Activation of SIRT1 in Hepatocytes Yang - 2019 - Molecular Nutrition \& Food Research - Wiley Online Library. (2020).

31. van de Weijer, T. et al. Lipotoxicity in type 2 diabetic cardiomyopathy. Cardiovascular Research 92, 10-18 (2020).

32. Stephens, N.A. et al. Intramyocellular lipid droplets increase with progression of cachexia in cancer patients. J Cachexia Sarcopenia Muscle 2, 111-117 (2011). 
33. Gray, C. et al. Magnetic resonance imaging with k-means clustering objectively measures whole muscle volume compartments in sarcopenia/cancer cachexia. Clin Nutr 30, 106-11 (2011).

34. Singh, J., Verma, N.K., Kansagra, S.M., Kate, B.N. \& Dey, C.S. Altered PPARgamma expression inhibits myogenic differentiation in $\mathrm{C} 2 \mathrm{C} 12$ skeletal muscle cells. Mol Cell Biochem 294, 163-71 (2007).

35. Hannafon, B.N. et al. Plasma exosome microRNAs are indicative of breast cancer. Breast Cancer Res 18, 90 (2016).

36. Tozzoli, R., D'Aurizio, F., Falcomer, F., Basso, S.M. \& Lumachi, F. Serum Tumor Markers in Stage I-II Breast Cancer. Med Chem 12, 285-9 (2016).

37. Nunez, C. Blood-based protein biomarkers in breast cancer. Clin Chim Acta 490, 113-127 (2019).

38. Delimaris, I. et al. Oxidized LDL, serum oxidizability and serum lipid levels in patients with breast or ovarian cancer. Clin Biochem 40, 1129-34 (2007).

39. Schwarzenbach, H. Clinical Relevance of Circulating, Cell-Free and Exosomal microRNAs in Plasma and Serum of Breast Cancer Patients. Oncol Res Treat 40, 423-429 (2017).

40. Amirfakhri, S., Salimi, A. \& Fernandez, N. Effects of Conditioned Medium from Breast Cancer Cells on TIr2 Expression in Nb4 Cells. - PubMed - NCBI. Asian Pac J Cancer Prev 16, 8445-50 (2015).

41. Guo, J. et al. Conditioned Medium from Malignant Breast Cancer Cells Induces an EMT-Like Phenotype and an Altered N-Glycan Profile in Normal Epithelial MCF10A Cells. in Int J Mol Sci, Vol. 18 (2017). 
42. Wessels, D.J. et al. Reciprocal signaling and direct physical interactions between fibroblasts and breast cancer cells in a 3D environment. PLoS One 14, e0218854 (2019).

43. Sousa, S. et al. Human breast cancer cells educate macrophages toward the M2 activation status. Breast Cancer Res 17, 101 (2015).

44. Luengo-Gil, G. et al. Angiogenic role of miR-20a in breast cancer. PLoS One 13, e0194638 (2018).

45. Furlan, A. et al. Ets-1 drives breast cancer cell angiogenic potential and interactions between breast cancer and endothelial cells. Int J Oncol 54, 2940 (2019).

46. Chen, D. et al. Cancer affects microRNA expression, release, and function in cardiac and skeletal muscle. Cancer Res 74, 4270-81 (2014).

47. Bates, J.P., Derakhshandeh, R., Jones, L. \& Webb, T.J. Mechanisms of immune evasion in breast cancer. in BMC Cancer, Vol. 18 (2018).

48. Gatti-Mays, M.E. et al. If we build it they will come: targeting the immune response to breast cancer. npj Breast Cancer 5, 1-13 (2019).

49. Fearon, K. et al. Definition and classification of cancer cachexia: an international consensus. Lancet Oncol 12, 489-95 (2011).

50. Fearon, K.C. et al. Definition of cancer cachexia: effect of weight loss, reduced food intake, and systemic inflammation on functional status and prognosis. The American Journal of Clinical Nutrition 83, 1345-1350 (2006).

51. Guigni, B.A., van der Velden, J., Kinsey, C.M., Carson, J.A. \& Toth, M.J. Effects of conditioned media from murine lung cancer cells and human tumor 
cells on cultured myotubes. Am J Physiol Endocrinol Metab 318, E22-e32 (2020).

52. Bohlen, J. et al. Dysregulation of metabolic-associated pathways in muscle of breast cancer patients: preclinical evaluation of interleukin-15 targeting fatigue. J Cachexia Sarcopenia Muscle (2018).

53. Werman, A. et al. Ligand-independent activation domain in the $\mathrm{N}$ terminus of peroxisome proliferator-activated receptor gamma (PPARgamma). Differential activity of PPARgamma1 and -2 isoforms and influence of insulin. J Biol Chem 272, 20230-5 (1997).

54. Jiang, X., Ye, X., Guo, W., Lu, H. \& Gao, Z. Inhibition of HDAC3 promotes ligand-independent PPARgamma activation by protein acetylation. J Mol Endocrinol 53, 191-200 (2014).

55. Hurtado, O. et al. Daidzein has neuroprotective effects through ligandbinding-independent PPARgamma activation. Neurochem Int 61, 119-27 (2012).

56. Al-Rasheed, N.M., Chana, R.S., Baines, R.J., Willars, G.B. \& Brunskill, N.J. Ligand-independent activation of peroxisome proliferator-activated receptorgamma by insulin and C-peptide in kidney proximal tubular cells: dependent on phosphatidylinositol 3-kinase activity. J Biol Chem 279, 49747-54 (2004).

57. Daniel, B. et al. The Nuclear Receptor PPARgamma Controls Progressive Macrophage Polarization as a Ligand-Insensitive Epigenomic Ratchet of Transcriptional Memory. Immunity 49, 615-626.e6 (2018). 
58. Brons, C. \& Grunnet, L.G. MECHANISMS IN ENDOCRINOLOGY: Skeletal muscle lipotoxicity in insulin resistance and type 2 diabetes: a causal mechanism or an innocent bystander? Eur J Endocrinol 176, R67-r78 (2017).

59. Bergman, B.C. et al. Intramuscular Lipid Metabolism in the Insulin Resistance of Smoking. in Diabetes, Vol. 58 2220-7 (2009).

60. Hevener, A.L. et al. Muscle-specific Pparg deletion causes insulin resistance. Nat Med 9, 1491-7 (2003).

61. Chavez, J.A. \& Summers, S.A. Characterizing the effects of saturated fatty acids on insulin signaling and ceramide and diacylglycerol accumulation in 3T3-L1 adipocytes and C2C12 myotubes. Arch Biochem Biophys 419, 101-9 (2003).

62. Rindler, P.M., Crewe, C.L., Fernandes, J., Kinter, M. \& Szweda, L.I. Redox regulation of insulin sensitivity due to enhanced fatty acid utilization in the mitochondria. Am J Physiol Heart Circ Physiol 305, H634-43 (2013).

63. Morales, P.E., Bucarey, J.L. \& Espinosa, A. Muscle Lipid Metabolism: Role of Lipid Droplets and Perilipins. J Diabetes Res 2017, 1789395 (2017).

64. Sarparanta, J., Garcia-Macia, M. \& Singh, R. Autophagy and Mitochondria in Obesity and Type 2 Diabetes. Curr Diabetes Rev 13, 352-369 (2017). 
CHAPTER 6: Discussion

Development of and insights from the BC-PDOX model of BC-related fatigue.

Previous animal model systems of CRF have been inadequate for the study of BCrelated fatigue, where women experience a significant degree of fatigue despite remaining weight stable. The lack of animal model systems that show fatigue in the absence of atrophy is likely a consequence of the commonly accepted assumption that the mechanisms underlying muscle fatigue in cancer are the same mechanisms that underlie muscle atrophy. Our work using CMA is significant as it helps to undermine this assumption, showing that the increased fatigability seen in two models of BC is likely not mediated by changes in muscle mass. This finding suggests that the mechanisms underlying muscle atrophy and fatigue are either independent of one another, or that fatigue occurs prior to atrophy. In either case, it is quite unlikely that targeting muscle atrophy would ameliorate fatigue. This fact highlights the importance of identifying model systems that show increased fatigability in the absence of muscle atrophy, and quantifying muscle function in addition to overall bodyweight when studying cancer cachexia.

Our work resulted in the important finding that the PDOX model of BC does recapitulate the clinically relevant phenotype of increased fatigability in the absence of atrophy, and we have utilized this model to uncover multiple potential mechanisms underlying this syndrome. Many of the pathways altered in the skeletal muscle of this model overlap with those that have been identified as contributors to cancer cachexia. This may indicate that these pathways underlie CRF that is typically observed in cancer cachexia rather than the atrophy component of the syndrome. Alternatively, these pathways may 
underlie muscle atrophy, but the relative dysregulation of these pathways in $B C$ is somehow too minor to allow for a phenotypic change in muscle mass.

Physiologically, our data indicate that slowed calcium release and reuptake in the PDOX skeletal muscle contributes to the increased fatigability observed, with PDOX muscle showing altered expression of multiple key regulators of calcium flow, including calsequestrin 1 , calsequestrin 2 , and the ryanodine receptor. This same phenotype of increased fatigability with alterations in calcium handling and increased relaxation times has been shown in diabetes mellitus (DM) ${ }^{57,58}$. Like women with $B C$, patients with DM experience an unusual degree of fatigue that is multi-faceted, poorly understood, and currently lacks targeted therapies ${ }^{59}$. This parallel in muscle physiology with DM suggests that $\mathrm{BC}$ may be inducing an insulin resistant phenotype in skeletal muscle. This possibility is supported by previous studies showing that women with $B C$ are more likely than other women to develop DM ${ }^{60}$.

The coordinated release and reuptake of calcium in skeletal muscle in response to an action potential is highly reliant on ATP availability, as calcium reuptake into the sarcoplasmic reticulum is an ATP-dependent process that must occur to allow for subsequent muscle contractions ${ }^{61}$. The slowing of this process then may reflect a decrease in ATP availability within muscle cells, which we confirmed by showing a decreased concentration of ATP in the mitochondria of PDOX-bearing animals' skeletal muscle. Decreased ATP availability could be reflective of numerous changes in skeletal muscle, including decreased storage of high-energy phosphate, decreased production of ATP by oxidative phosphorylation or glycolytic processes, or increased utilization of ATP. Our analysis of the skeletal muscle transcriptome in the PDOX mice suggests that 
the production of ATP by oxidative processes may be disrupted due to downregulation of genes involved in mitochondrial biogenesis and fatty acid metabolism, and the utilization of ATP may be increased in inflammatory processes. Improving metabolic function may therefore be a useful cognate therapy to improve ATP availability in skeletal muscle. One possible avenue to improve metabolic function could be to utilize agonists of the PPAR family of proteins, which have demonstrated roles in regulating whole body energy homeostasis ${ }^{49}$, have multiple synthetic and endogenous agonists 62, and are regulators of a significant portion of the genes downregulated in BC-PDOX skeletal muscle relative to control. PPARY agonists have been utilized in clinical trials for BC previously, as it was hypothesized that the agonists would induce terminal differentiation in $\mathrm{BC}$ cells ${ }^{63,64}$. Though no clinical benefit was established in terms of BC progression in a phase II trial with the PPARy ligand troglitazone, the study did not report assessments of body weight, muscle strength, or muscle fatigue, and the drug was tolerated well by patients ${ }^{65}$. Because PPARy agonists are currently approved for the treatment of DM due to their action as insulin sensitizers, if insulin resistance is the underlying phenotype responsible for BC patients' increased fatigability, PPARY agonists or other insulin sensitizing agents could aid in ameliorating CRF by restoring insulin sensitivity.

Clinical correlates of skeletal muscle molecular profiles in BC.

We found that women with three molecular subtypes of BC, those being ERPR, TN, and TP BC, exhibit overall similarity in muscular gene expression, with a gene expression profile that is quite distinct from that observed in women with tumors overexpressing HER2/neu in the absence of ER and PR. Despite this marked difference in gene 
expression, all subtypes showed similar pathway-level dysregulation, indicating that treatment methods for muscle fatigue in $\mathrm{BC}$ patients may be generalizable across molecular subtypes of BC. Repression of transcripts involved in several metabolic pathways, including pathways regulating mitochondrial functions as well as carbohydrate, lipid, and protein metabolism, was consistently observed across all subtypes. Additionally, we observed downregulation of electron transport chain (ETC) proteins in muscle from patients with HER2/neu-overexpressing tumors. As previous publications have shown that abundance of ETC complex proteins strongly correlates with skeletal muscle oxidative capacity and mitochondrial density ${ }^{66}, \mathrm{BC}$ patients likely also have decreased mitochondrial density in their skeletal muscle and decreased oxidative capacity.

One particularly interesting finding from our work was that the molecular changes occurring in skeletal muscle in response to $\mathrm{BC}$ appear to be relatively independent of treatment history, indicating that the muscle-intrinsic mechanisms of CRF are not a response to anti-cancer therapies. This hypothesis is supported by our in vitro conditioned media experiments where tumor-derived factors directly repressed aerobic ATP production and PPAR-mediated transcription in differentiated muscle cells.

Additional interesting findings from assessing clinical data against skeletal muscle gene expression data included the lack of a relationship between gene expression and body weight change, and the near statistically significant relationship between gene expression and serum albumin. These findings suggest that skeletal muscle gene expression changes reported in our work are not reflective of a cachectic process, and that serum albumin could be a useful biomarker for muscle dysfunction, although 
prospective studies would be required to assess these possibilities. Our laboratory plans to address this in a new cohort of patients, and the study design is currently under development.

In vitro modeling of $B C$-induced skeletal muscle dysfunction

Our in vitro work clearly demonstrates that BC-derived factors can significantly modulate function of skeletal muscle cells, and that this crosstalk does not require the $\mathrm{BC}$ cells to be treated with any anti-cancer therapies or the involvement of immune or stromal cells. Rather, miRNAs, exosomes, proteins, or other small molecules could mediate this crosstalk. This finding importantly contradicts recent reports in other cancer types, where CM from cancer cells did not induce skeletal muscle mitochondrial deficits in vitro ${ }^{67}$. This contrast suggests that different cancer types induce skeletal muscle dysfunction via different mechanisms, or perhaps that the small methodological differences between the two laboratories is responsible for the difference in results.

Muscle fatigue is a mechanistically complicated process and can result from a myriad of factors. In general though, the underlying ability of muscle maintain force production in response to stimuli relies upon the muscle's ability to resynthesize ATP following stimulus. Our in vivo data clearly demonstrate mitochondrial dysfunction in muscle of patients with $\mathrm{BC}$ and in BC-PDOX mice, which would directly impact the fatigability of skeletal muscle by reducing the muscle's ability to maintain adequate high-energy phosphates required for repeated contractions. Our in vitro data then expand on these findings and suggest this mitochondrial dysfunction is a direct result of BC-derived factors. Interestingly, the observed mitochondrial dysfunction is accompanied by increased intracellular lipid deposition, though these processes appear to be 
mechanistically separate from one another. Intramuscular lipid deposition has also been reported in many pathologies other than cancer, including type 2 diabetes ${ }^{68}$ and smoking-induced insulin resistance ${ }^{69}$. As PPARG expression and transcriptional activity are associated with insulin resistance ${ }^{50}$ and many PPAR transcriptional targets are mitochondrial lipid transport proteins ${ }^{70}$, we propose that alterations in mitochondrial lipid import may be at least partially responsible for the observed lipid accumulation in response to BC-derived factors. Future studies should investigate this potential link in greater detail.

Our development of an in vitro model system to study BC-induced skeletal muscle dysfunction is significant for several reasons. First, we have reported the first successful attempt to induce metabolic changes in skeletal muscle in an in vitro conditioned media system. Additionally, we present the finding that the metabolic effects of $B C$ on skeletal muscle are at least partially mediated via PPAR signaling pathways. This finding provides rationale for investigating PPAR agonists to improve quality of life in survivors of $\mathrm{BC}$, and also provides support for the translational relevance of the in vitro system given the similarities to phenotypes observed in $\mathrm{BC}$-induced skeletal muscle fatigue in mouse models of $\mathrm{BC}$ and in women with $\mathrm{BC}$.

\section{Limitations and future directions}

The most significant limitation in our in vivo work is the fact that our RNA-seq and proteomic analyses in human subjects did not include any measure of fatigue. Thus, we cannot say with certainty that the molecular changes observed in skeletal muscle of BC patients are in fact reflective of increased fatigability. However, our data certainly show that BC-PDOX mice exhibit higher rates of fatigue, and we also know from current 
literature that the vast majority of women with BC experience CRF. Therefore, it is likely that the molecular changes occurring in both the BC-PDOX muscle and human muscle are reflective of the mechanisms underlying fatigue in human patients. Our laboratory is currently developing a new protocol that will include obtaining subjective and/or objective measures of fatigue in patients to assess alongside skeletal muscle molecular changes induced by BC. Once we are certain that our targeted mechanisms are indeed reflective of CRF, interventional studies targeting these pathways would be appropriate.

A similar limitation is also apparent in our in vitro assays. While our data strongly support the idea that PPARG activity is somehow involved in the mitochondrial dysfunction and lipid accumulation induced by BC-derived factors, we cannot say whether or not these phenotypes are reflective of fatigability in vivo. Mechanistically, it is reasonable to suppose a causal link between defects in metabolic functions and fatigability, particularly in light of the fact that both the BC-PDOX mice and BC patients showed decreased ATP content in their muscle tissue relative to control. However, a reasonable supposition is still only a supposition, and creative methodologies will be required to definitively connect metabolic dysfunction in vitro to fatigability in vivo.

\section{Overall conclusions}

Our laboratory's work has culminated in several important findings. First, the identification of the BC-PDOX model as an appropriate model for studying BC-induced muscle dysfunction in the absence of cachexia is of paramount importance to the study of this common and debilitating condition. Targeting muscle dysfunction in $\mathrm{BC}$ patients has the potential to improve the lives of millions of $B C$ survivors, and to potentially improve overall survival in women undergoing treatments. Further, utilizing RNA-seq 
and proteomic analyses, we show that BC patients' skeletal muscle exhibits dysregulation of multiple metabolic pathways regardless of treatment history, molecular subtype, body composition, or changes in bodyweight, with dysregulation of oxidative phosphorylation and signaling via the PPAR family of proteins overlapping between all molecular subtypes of BC and BC-PDOX mice. Dysregulation of these pathways could explain the significant reduction in ATP content seen in mitochondria and bulk muscle tissue from BC-PDOX mice and BC patients, respectively. Our in vitro work establishes an important model system for studying the metabolic effects of BC-derived factors on differentiated skeletal muscle, shows that BC-derived factors can directly modulate skeletal muscle function without the involvement of immune or stromal cell mediators, and shows that signaling via the PPAR family is likely integral to these effects. Overall, our findings point to significant metabolic dysregulation in skeletal muscle as a main contributor to the increased fatigability seen in BC-PDOX mice and women with BC, with these effects being induced directly by $\mathrm{BC}$-derived factors rather than via immunological mediators or as adverse effects of therapy. 


\section{BIBLIOGRAPHY}

1. de Moor JS, Mariotto AB, Parry C, et al. Cancer survivors in the United States: prevalence across the survivorship trajectory and implications for care. Cancer Epidemiol Biomarkers Prev. 2013;22: 561-570.

2. Yoshimura A, Ito $\mathrm{H}$, Nishino $\mathrm{Y}$, et al. Recent Improvement in the Long-term Survival of Breast Cancer Patients by Age and Stage in Japan. J Epidemiol, 2018:420-427.

3. Narod S, Iqbal J, Miller A. Why have breast cancer mortality rates declined? J Cancer Policy. 2015;5: 8-17.

4. Survival Rates for Breast Cancer. Available from URL:

https://www.cancer.org/cancer/breast-cancer/understanding-a-breast-cancerdiagnosis/breast-cancer-survival-rates.html [accessed 3 March 2020.

5. Escalante CP, Manzullo EF. Cancer-Related Fatigue: The Approach and Treatment. J Gen Intern Med, 2009:412-416.

6. Blesch KS, Paice JA, Wickham R, et al. Correlates of fatigue in people with breast or lung cancer. Oncol Nurs Forum. 1991;18: 81-87.

7. Bower JE, Ganz PA, Desmond KA, Rowland JH, Meyerowitz BE, Belin TR. Fatigue in breast cancer survivors: occurrence, correlates, and impact on quality of life. J Clin Oncol. 2000;18: 743-753. 
8. Cella D, Davis K, Breitbart W, Curt G, Coalition F. Cancer-related fatigue: prevalence of proposed diagnostic criteria in a United States sample of cancer survivors. J Clin Oncol. 2001;19: 3385-3391.

9. Curt GA, Breitbart W, Cella D, et al. Impact of cancer-related fatigue on the lives of patients: new findings from the Fatigue Coalition. Oncologist. 2000;5: 353-360.

10. Ancoli-Israel S, Liu L, Marler MR, et al. Fatigue, sleep, and circadian rhythms prior to chemotherapy for breast cancer. Support Care Cancer. 2006;14: 201-209.

11. Cimprich B. Pretreatment symptom distress in women newly diagnosed with breast cancer. Cancer Nurs. 1999;22: 185-194; quiz 195.

12. Evans WJ, Morley JE, Argilés J, et al. Cachexia: a new definition. Clin Nutr. 2008;27: 793-799.

13. Cella D, Lai JS, Chang $\mathrm{CH}$, Peterman A, Slavin M. Fatigue in cancer patients compared with fatigue in the general United States population. Cancer. 2002;94: 528538.

14. Ganz PA, Bower JE. Cancer related fatigue: a focus on breast cancer and Hodgkin's disease survivors. Acta Oncol. 2007;46: 474-479.

15. Arndt V, Stegmaier $\mathrm{C}$, Ziegler $\mathrm{H}$, Brenner $\mathrm{H}$. A population-based study of the impact of specific symptoms on quality of life in women with breast cancer 1 year after diagnosis. Cancer. 2006;107: 2496-2503. 
16. Peters KB, West MJ, Hornsby WE, et al. Impact of health-related quality of life and fatigue on survival of recurrent high-grade glioma patients. J Neurooncol. 2014;120: 499-506.

17. Wang XS, Woodruff JF. Cancer-related and treatment-related fatigue. Gynecol Oncol. 2015;136: 446-452.

18. Groenvold M, Petersen MA, Idler E, Bjorner JB, Fayers PM, Mouridsen HT. Psychological distress and fatigue predicted recurrence and survival in primary breast cancer patients. Breast Cancer Res Treat. 2007;105: 209-219.

19. Prado CM, Baracos VE, McCargar LJ, et al. Sarcopenia as a determinant of chemotherapy toxicity and time to tumor progression in metastatic breast cancer patients receiving capecitabine treatment. Clin Cancer Res. 2009;15: 2920-2926.

20. Escalante C. Cancer-related fatigue: Treatment - UpToDate. Available from URL: https://www.uptodate.com/contents/cancer-related-fatigue-treatment?search=cancer related fatigue treatment\&source=search result\&selectedTitle=1 30\&usage_type=default\&display_ran $k=1-H 10$ 2019].

21. Bower JE. Cancer-related fatigue--mechanisms, risk factors, and treatments. Nat Rev Clin Oncol. 2014;11: 597-609.

22. Bardwell WA, Ancoli-Israel S. Breast Cancer and Fatigue. Sleep Med Clin. 2008;3: $61-71$. 
23. Shakeel S, School of Medicine UoT, Toronto, Ontario, Canada, Tung J, et al. Evaluation of Factors Associated With Unmet Needs in Adult Cancer Survivors in Canada. JAMA Network Open. 2020;3.

24. Fearon K, Strasser F, Anker SD, et al. Definition and classification of cancer cachexia: an international consensus. Lancet Oncol. 2011;12: 489-495.

25. NOT-CA-20-008: Notice of Intent to Publish a Funding Opportunity Announcement for Research Answers to NCls Provocative Questions. Available from URL: https://grants.nih.gov/grants/guide/notice-files/NOT-CA-20-008.html.

26. Vanhoutte G, van de Wiel M, Wouters K, et al. Cachexia in cancer: what is in the definition? BMJ Open Gastroenterol. 2016;3: e000097.

27. Martin L, Birdsell L, Macdonald N, et al. Cancer cachexia in the age of obesity: skeletal muscle depletion is a powerful prognostic factor, independent of body mass index. J Clin Oncol. 2013;31: 1539-1547.

28. Baracos VE, Martin L, Korc M, Guttridge DC, Fearon KCH. Cancer-associated cachexia. Nat Rev Dis Primers. 2018;4: 17105.

29. Crawford J. What are the criteria for response to cachexia treatment? Ann Palliat Med. 2019;8: 43-49.

30. Bozzetti F, Mariani L. Defining and classifying cancer cachexia: a proposal by the SCRINIO Working Group. JPEN J Parenter Enteral Nutr. 2009;33: 361-367. 
31. Fearon KC, From the Royal Infirmary of Edinburgh E, United Kingdom (KCF), and the Ross Products Division, Abbott Laboratories, Columbus, OH (ACV and DSH), Voss AC, From the Royal Infirmary of Edinburgh E, United Kingdom (KCF), and the Ross Products Division, Abbott Laboratories, Columbus, $\mathrm{OH}$ (ACV and DSH), Hustead DS, From the Royal Infirmary of Edinburgh E, United Kingdom (KCF), and the Ross Products Division, Abbott Laboratories, Columbus, $\mathrm{OH}$ (ACV and DSH). Definition of cancer cachexia: effect of weight loss, reduced food intake, and systemic inflammation on functional status and prognosis. The American Journal of Clinical Nutrition. 2006;83: $1345-1350$.

32. KATZ AM, KATZ PB. Diseases of the heart in the works of Hippocrates. Br Heart J. 1962;24: 257-264.

33. Kilgour RD, Vigano A, Trutschnigg B, et al. Cancer-related fatigue: the impact of skeletal muscle mass and strength in patients with advanced cancer. J Cachexia Sarcopenia Muscle. 2010;1: 177-185.

34. Gray C, MacGillivray TJ, Eeley C, et al. Magnetic resonance imaging with k-means clustering objectively measures whole muscle volume compartments in sarcopenia/cancer cachexia. Clin Nutr. 2011;30: 106-111.

35. Stephens NA, Gray C, MacDonald AJ, et al. Sexual dimorphism modulates the impact of cancer cachexia on lower limb muscle mass and function. Clin Nutr. 2012;31: 499-505. 
36. Neefjes ECW, van den Hurk RM, Blauwhoff-Buskermolen S, et al. Muscle mass as a target to reduce fatigue in patients with advanced cancer. J Cachexia Sarcopenia Muscle. 2017;8: 623-629.

37. Fox KM, Brooks JM, Gandra SR, Markus R, Chiou CF. Estimation of Cachexia among Cancer Patients Based on Four Definitions. J Oncol. 2009;2009: 693458.

38. Del Fabbro E, Dev R, Hui D, Palmer L, Bruera E. Effects of melatonin on appetite and other symptoms in patients with advanced cancer and cachexia: a double-blind placebo-controlled trial. J Clin Oncol. 2013;31: 1271-1276.

39. Jatoi A, Dakhil SR, Nguyen PL, et al. A placebo-controlled double blind trial of etanercept for the cancer anorexia/weight loss syndrome: results from N00C1 from the North Central Cancer Treatment Group. Cancer. 2007;110: 1396-1403.

40. Kanat O, Cubukcu E, Avci N, et al. Comparison of three different treatment modalities in the management of cancer cachexia. Tumori. 2013;99: 229-233.

41. Strasser F, Luftner D, Possinger K, et al. Comparison of orally administered cannabis extract and delta-9-tetrahydrocannabinol in treating patients with cancerrelated anorexia-cachexia syndrome: a multicenter, phase III, randomized, double-blind, placebo-controlled clinical trial from the Cannabis-In-Cachexia-Study-Group. J Clin Oncol. 2006;24: 3394-3400.

42. Jatoi A, Ritter HL, Dueck A, et al. A placebo-controlled, double-blind trial of infliximab for cancer-associated weight loss in elderly and/or poor performance nonsmall cell lung cancer patients (N01C9). Lung Cancer. 2010;68: 234-239. 
43. Temel JS, Abernethy AP, Currow DC, et al. Anamorelin in patients with non-smallcell lung cancer and cachexia (ROMANA 1 and ROMANA 2): results from two randomised, double-blind, phase 3 trials. Lancet Oncol. 2016;17: 519-531.

44. Shukla SK, Singh PK. Chapter 3 - Animal models of cancer-associated cachexia. In: Azmi A, Mohammad R, editors. Animal Models in Cancer Drug Discovery: Academic Press, 2019.

45. Zombeck JA, Fey EG, Lyng GD, Sonis ST. A clinically translatable mouse model for chemotherapy-related fatigue. Comp Med. 2013;63: 491-497.

46. Dougherty JP, Wolff BS, Cullen MJ, Saligan LN, Gershengorn MC. Taltirelin alleviates fatigue-like behavior in mouse models of cancer-related fatigue. Pharmacol Res. 2017;124: 1-8.

47. Hoffman RM. Patient-derived orthotopic xenografts: better mimic of metastasis than subcutaneous xenografts. Nat Rev Cancer. 2015;15: 451-452.

48. Allen DG, Lamb GD, Westerblad H. Skeletal muscle fatigue: cellular mechanisms. Physiol Rev. 2008;88: 287-332.

49. Berger J, Moller DE. The mechanisms of action of PPARs. Annu Rev Med. 2002;53: 409-435.

50. Hevener AL, He W, Barak Y, et al. Muscle-specific Pparg deletion causes insulin resistance. Nat Med. 2003;9: 1491-1497. 
51. Chiarelli F, Di Marzio D. Peroxisome proliferator-activated receptor-gamma agonists and diabetes: current evidence and future perspectives. Vasc Health Risk Manag. 2008;4: 297-304.

52. Moore-Carrasco R, Figueras M, Ametller E, López-Soriano FJ, Argilés JM, Busquets S. Effects of the PPARgamma agonist GW1929 on muscle wasting in tumourbearing mice. Oncol Rep. 2008;19: 253-256.

53. Jiang F, Zhang Z, Zhang Y, Pan X, Yu L, Liu S. L-Carnitine Ameliorates Cancer Cachexia in Mice Partly via the Carnitine Palmitoyltransferase-Associated PPAR- $\gamma$ Signaling Pathway. Oncol Res Treat. 2015;38: 511-516.

54. Goncalves MD, Hwang SK, Pauli C, et al. Fenofibrate prevents skeletal muscle loss in mice with lung cancer. Proc Natl Acad Sci U S A. 2018;115: E743-E752.

55. Wilson HE, Rhodes KK, Rodriguez D, et al. Human breast cancer xenograft model implicates peroxisome proliferator-activated receptor signaling as driver of cancerinduced muscle fatigue. Clinical Cancer Research. 2018: clincanres.1565.2018.

56. Zhao S, Li C-I, Guo Y, Sheng Q, Shyr Y. RnaSeqSampleSize: RnaSeqSampleSize. .

57. Eshima H, Poole DC, Kano Y. In vivo calcium regulation in diabetic skeletal muscle. Cell Calcium. 2014;56: 381-389. 
58. Eshima $\mathrm{H}$, Tanaka $\mathrm{Y}$, Sonobe $\mathrm{T}$, et al. In vivo imaging of intracellular $\mathrm{Ca} 2+$ after muscle contractions and direct $\mathrm{Ca} 2+$ injection in rat skeletal muscle in diabetes. Am J Physiol Regul Integr Comp Physiol. 2013;305: R610-618.

59. Kalra S, Sahay R. Diabetes Fatigue Syndrome. Diabetes Ther, 2018:1421-1429.

60. Lipscombe LL, Chan WW, Yun L, Austin PC, Anderson GM, Rochon PA. Incidence of diabetes among postmenopausal breast cancer survivors. Diabetologia. 2013;56: 476-483.

61. Primeau JO, Armanious GP, Fisher ME, Young HS. The SarcoEndoplasmic Reticulum Calcium ATPase. Subcell Biochem. 2018;87: 229-258.

62. Grygiel-Górniak B. Peroxisome proliferator-activated receptors and their ligands: nutritional and clinical implications - a review. Nutrition Journal. 2014;13: 1-10.

63. Fenner MH, Elstner E. Peroxisome proliferator-activated receptor-gamma ligands for the treatment of breast cancer. Expert Opin Investig Drugs. 2005;14: 557-568.

64. Mueller E, Sarraf $P$, Tontonoz $P$, et al. Terminal differentiation of human breast cancer through PPAR gamma. Mol Cell. 1998;1: 465-470.

65. Burnstein HJ, Demetri GD, Mueller E, Sarraf P, Spiegelman BM, Winer EP. Use of the peroxisome proliferator-acivated receptor gamma ligand troglitazone as treatment for refractory breast cancer: a phase II study. Breast Cancer Research and Treatment. 2003;79: 391-397. 
66. Larsen S, Nielsen J, Hansen CN, et al. Biomarkers of mitochondrial content in skeletal muscle of healthy young human subjects. J Physiol. 2012;590: 3349-3360.

67. Guigni BA, van der Velden J, Kinsey CM, Carson JA, Toth MJ. Effects of conditioned media from murine lung cancer cells and human tumor cells on cultured myotubes. Am J Physiol Endocrinol Metab. 2020;318: E22-e32.

68. Brons C, Grunnet LG. MECHANISMS IN ENDOCRINOLOGY: Skeletal muscle lipotoxicity in insulin resistance and type 2 diabetes: a causal mechanism or an innocent bystander? Eur J Endocrinol. 2017;176: R67-r78.

69. Bergman BC, Perreault L, Hunerdosse DM, Koehler MC, Samek AM, Eckel RH. Intramuscular Lipid Metabolism in the Insulin Resistance of Smoking. Diabetes, 2009:2220-2227.

70. Fang L, Zhang M, Li Y, Liu Y, Cui Q, Wang N. PPARgene: A Database of Experimentally Verified and Computationally Predicted PPAR Target Genes. PPAR Res. 2016;2016: 6042162. 